\title{
A Derivation of Linearized Griffith Energies from Nonlinear Models
}

\author{
MANUEL FRIEDRICH
}

Communicated by G. M. OrTIz

\begin{abstract}
We derive Griffith functionals in the framework of linearized elasticity from nonlinear and frame indifferent energies in a brittle fracture via $\Gamma$-convergence. The convergence is given in terms of rescaled displacement fields measuring the distance of deformations from piecewise rigid motions. The configurations of the limiting model consist of partitions of the material, corresponding piecewise rigid deformations and displacement fields which are defined separately on each component of the cracked body. Apart from the linearized Griffith energy the limiting functional also comprises the segmentation energy, which is necessary to disconnect the parts of the specimen.
\end{abstract}

\section{Contents}

1. Introduction . . . . . . . . . . . . . . . . . . . 426

2. The Model and Main Results . . . . . . . . . . . . . . . . . . . . . . . . 4830

2.1. The Nonlinear Model . . . . . . . . . . . . . . . . . . . . . . . . . . . 430

2.2. The Segmentation Problem . . . . . . . . . . . . . . . . . . . 431

2.3. Compactness and Limiting Configurations . . . . . . . . . . . . . . . . . 432

2.4. The Limiting Linearized Model and $\Gamma$-Convergence . . . . . . . . . . . . . 435

2.5. Application: External Loads . . . . . . . . . . . . . . . . . . . . . . . . . 437

2.6. Application: Cleavage Laws . . . . . . . . . . . . . . . . . . . 438

3. Preliminaries . . . . . . . . . . . . . . . . . . . . . . . . 439

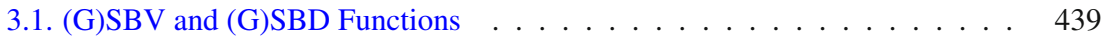

3.2. Caccioppoli Partitions . . . . . . . . . . . . . . . . . . . . . . . 442

3.3. Rigidity Estimates . . . . . . . . . . . . . . . . . . . . . . . 443

4. Compactness of Rescaled Configurations . . . . . . . . . . . . . . . . . . 445

4.1. Preparations . . . . . . . . . . . . . . . . . . . . 445

4.2. Proof of Theorem $2.2 \ldots \ldots \ldots$. . . . . . . . . . . . . . . . 446

5. Admissible and Coarsest Partitions and Limiting Configurations . . . . . . . . . 453

5.1. Equivalent Characterization of the Coarsest Partition . . . . . . . . . . . . 454

5.2. Admissible Configurations . . . . . . . . . . . . . . . 456 
5.3. Existence of Coarsest Partitions . . . . . . . . . . . . . . . . . . . . . 457

6. The Effective Linearized Griffith Model . . . . . . . . . . . . . . . . . . . . . . 460

6.1. Derivation of Linearized Models via $\Gamma$-Convergence . . . . . . . . . . . . . . . 460

6.2. An Application to Cleavage Laws . . . . . . . . . . . . . . . . . . . . . 463

References . . . . . . . . . . . . . . . . . . . . . . . . 466

\section{Introduction}

A thorough understanding of crack formation in brittle materials is of great interest in both experimental sciences and theoretical studies. Starting with the seminal contribution by FRANCFORT AND MARIGO [27], where the displacements and crack paths were determined from an energy minimization principle, various variational models in the framework of free discontinuity problems have appeared in the literature over the past years. These so-called Griffith functionals, comprising elastic and surface contributions, generalize the original Griffith theory (see [33]) which is based on the fundamental idea that the formation of fracture may be regarded as the competition of elastic bulk and surface energies.

For the sake of a simplified mathematical description the investigation of fracture models in the realm of linearized elasticity is widely adopted (see for example $[2,4,7,13,14,34])$ and has led to a lot of realistic applications in engineering as well as to efficient numerical approximation schemes (we refer to $[5,6,12,26,38,39,43]$ making no claim to be exhaustive). On the contrary, their nonlinear counterparts are usually significantly more difficult to treat since in the regime of finite elasticity the energy density of the elastic contributions is genuinely geometrically nonlinear due to frame indifference rendering the problem highly non-convex. Consequently, in contrast to linear models, already the fundamental question of whether minimizing configurations for given boundary data exist at all is a major difficulty. An even more challenging task in this context is the determination of the material behavior under expansion or compression, in particular the derivation of specific cleavage laws.

Consequently, for a deeper understanding of nonlinear models the identification of an effective linearized theory is desirable as in this way one may rigorously show that in the small displacement regime neglecting the effects arising from the nonlinearities is a good approximation of the problem. Moreover, such a derivation is also interesting in the context of discrete systems. Previous investigations which were motivated by the analysis of cleavage laws for brittle crystals (see [29,30] or the seminal paper [10]) have shown that the most interesting regime for the elastic strains is given by $\sqrt{\varepsilon}$, where $\varepsilon$ denotes the typical interatomic distance. Consequently, a passage from discrete-to-continuum systems naturally involves a simultaneous linearization process.

In elasticity theory the nonlinear-to-linear limit is by now well understood in various different settings via $\Gamma$-convergence (cf. $[11,21,41,42])$, where the passage is performed in terms of suitably rescaled displacement fields measuring the distance of the deformation from a rigid motion and being the fundamental quantity on which the linearized elastic energy depends. In fracture mechanics, however, the relation between the deformation of a material and corresponding displacements is 
more complicated since the body may be disconnected by the jump set into various components. In fact, it turns out that, without passing to rescaled configurations, in the small strain limit nonlinear Griffith energies converge to a limiting functional which is finite for piecewise rigid motions and measures the segmentation energy which is necessary to disconnect the body.

Obviously a major drawback of this simple limiting model appears to be the fact that it does not capture the elastic deformations which are typically present in the nonlinear models. Consequently, in order to arrive at a limiting model showing the coexistence of elastic and surface contributions it is indispensable to pass to rescaled configurations similarly as in [21]. The goal of this article is to identify such an effective linearized Griffith energy as the $\Gamma$-limit of nonlinear and frame indifferent models in the small strain regime. To the best of our knowledge such a result has not yet been derived in the general setting of free discontinuity problems introduced by Ambrosio AND De Giorgi [23].

The farthest reaching result in this direction seems to be a recent contribution by NEGRI AND TOADER [40] where a nonlinear-to-linear analysis is performed in the context of quasistatic evolution for a restricted class of admissible cracks. In particular, in their model, the different components of the jump set are supposed to have a least positive distance rendering the problem considerably easier from an analytical point of view. In particular, the specimen cannot be separated into different parts, effectively leading to a simple relation between the deformation and the rescaled displacement field. On the other hand, in [31] we have performed a simultaneous discrete-to-continuum and nonlinear-to-linear analysis for general crack geometries, but under the simplifying assumption that all deformations lie close to the identity mapping.

In the present context we establish a limiting linearized Griffith functional in a planar setting without any a priori assumptions on the deformation and the crack geometry. We identify an effective model which appears to be more general than the energies which are widely investigated in the literature. Whereas in elasticity theory, in the approaches [31,40] mentioned before and in most linear fracture models there is a simple relation between the deformation of the material and the associated infinitesimal displacement field, in our framework the deformation is related to a triple consisting of a partition of the domain, a corresponding piecewise rigid motion being constant on each connected component of the cracked body and a displacement field which is defined separately on each piece of the specimen.

On each component of the partition the energy is of Griffith-type in the realm of linearized elasticity. In addition, the functional contains the segmentation energy which is necessary to disconnected the parts of the body. In particular, the latter contribution is a specific feature of our general model where we do not restrict the analysis to a linearization around a fixed rigid motion.

Let us briefly note that although all arguments used in the proofs of this article are valid in any space dimension, we have to restrict our analysis to two dimensions as one of the ingredients of our analysis, an $S B D$-rigidity result (see [28]), has only been derived in a planar setting for isotropic surface energies. However, we believe that the estimate in [28] may be generalized in the future and then the generalizations for the results in the work at hand immediately follow. 
As applications of our result we investigate problems with external forces and also present a cleavage law in a continuum setting with isotropic surface energies. As discussed before, the identification of critical loads and the investigation of crack paths is a challenging problem particularly for nonlinear models. The arguments in $[29,30,37]$, where boundary value problems of uniaxial extension for brittle materials were investigated, fundamentally relied on the application of certain slicing techniques and due to the lack of convexity were not adapted to treat the case of compression. Our general $\Gamma$-limit result can now be applied to also solve boundary value problems of uniaxial compression which is, like the uniaxial tension test, a natural and interesting problem. Hereby we may complete the picture about the derivation of cleavage laws in [29,30].

One essential point in our investigation is the establishing of a compactness result providing limiting configurations which consist of piecewise rigid motions and corresponding displacement fields. Similarly to the derivation of linearized systems for elastic materials (see for example [21]), where the main ingredient is a quantitative geometric rigidity estimate by FRIESECKE ET AL. [32], the starting point of our analysis is a quantitative $S B D$-rigidity result (see [28]) in the framework of special functions of bounded deformation (see $[2,4]$ ), which is tailor-made for general Griffith models with coexistence of both energy forms.

As there is no uniform bound on the functions, it turns out that the limiting displacements are generically not summable and we naturally end up in the space of GSBD functions (for the definition and basic properties we refer to [18]). We believe that our results are interesting also outside of this specific context as they allow to solve more general variational problems in fracture mechanics. Typically, for compactness results in function spaces as $S B V$ (see [3] for the definition and basic properties) and $S B D$ one needs $L^{\infty}$ or $L^{1}$ bounds on the functions (see $[1,4,18]$ ). However, in many applications, in particular for atomistic systems and for models dealing with rescaled deformations, such estimates cannot be inferred from energy bounds. Nevertheless, we are able to treat problems without any a priori bound by passing from the deformations to displacement fields whose distance from rigid motions can be controlled.

The other essential point in our analysis is the investigation of the limiting configurations. In particular, we study the properties of the partition which disconnects the body into various parts. It turns out that an even finer segmentation may occur if on a connected component of the partition the jump set of the corresponding displacement field further separates the body. Here it becomes apparent that we treat a real multiscale model as the jump heights at the boundaries associated to the coarse partition are of order $\gg \sqrt{\varepsilon}(\sqrt{\varepsilon}$ denotes the regime of the typical elastic strain), whereas the jump heights of the finer partition are of order $\sqrt{\varepsilon}$. Moreover, it is evident that the choice of the limiting partition is not unique. However, we propose a selection principle and show the existence and uniqueness of a coaresest partition.

The paper is organized as follows. In Section 2 we state the main compactness and $\Gamma$-convergence results and discuss properties of the limiting linearized Griffith functional. Moreover, we present our application to cleavage laws for uniaxially extended or compressed brittle materials. 
Section 3 is devoted to some preliminaries. We first give the definition of special functions of bounded variation and deformation and discuss basic properties. Afterwards, we recall the notion of Caccioppoli partitions which will be fundamental in our analysis to analyze the properties of limiting configurations. Moreover, we recall geometric rigidity results for elastic and brittle materials, in particular the $S B D$-rigidity result proved in [28].

In Section 4 we then establish the main compactness result for a sequence of deformations $\left(y_{\varepsilon}\right)_{\varepsilon}$, where $\varepsilon$ stands for the order of the elastic energy. First, the convergence of the partitions and the corresponding rigid motions is based on compactness theorems for Caccioppoli partitions and piecewise constant functions (see [3] or Section 3.2 below).

Although the $S B D$-rigidity estimate is a fundamental ingredient in our analysis giving $L^{2}$ bounds for rescaled displacement fields, we still have to face major difficulties since the rigidity estimate provides a family of displacement fields $\left(u_{\varepsilon}^{\rho}\right)_{\varepsilon}^{\rho}$ with an additional parameter $\rho$ representing a 'modification error' between $y_{\varepsilon}$ and $u_{\varepsilon}^{\rho}$. Consequently, the goal will be to choose an appropriate diagonal sequence.

An additional challenge is the fact that the bounds in the $S B D$-rigidity estimate depend on $\rho$ and blow up for $\rho \rightarrow 0$. For the symmetric part of the gradient this problem can be bypassed by a Taylor expansion taking the nonlinear elastic energy $\varepsilon$ and a higher order term into account, which shows that the constant may be chosen independently of $\rho$. For the function itself, however, the problem is more subtle since a uniform bound cannot be inferred by energies bounds. In particular, generically, the limiting configurations are not in $L^{2}$ but are only finite almost everywhere. The strategy to establish the latter assertion is to show that for fixed $\varepsilon$ the functions $\left(u_{\varepsilon}^{\rho}\right)_{\rho}$ essentially coincide in a certain sense on the bulk part of the domain. Afterwards, by a careful analysis, we can derive that such a property is preserved in the limit $\varepsilon \rightarrow 0$, whereby we can establish a kind of equi-integrability of the configurations.

In Section 5 we concern ourselves with the limiting configurations consisting of a partition, a corresponding piecewise rigid motion and a displacement field. Recalling that the limits provided by the compactness result are highly non-unique we introduce the notion of a coarsest partition. Roughly speaking, the definition states that the jump heights at the boundaries associated to this partition are of order $\gg \sqrt{\varepsilon}$ leading to a meaningful mathematical description of the observation that the size of the crack opening is a multiscale phenomenon in our model.

The fundamental point is the proof of the existence and uniqueness of the coarsest partition. Uniqueness follows from the fact that under the assumption that there are two different coarsest partitions one always can find an even coarser partition. Existence is a more challenging problem. We first give an alternative characterization and identify coarsest partitions as the maximal elements of the partial order on the set of admissible partitions which is induced by subordination. We then show that each chain of the partial order has an upper bound repeating some arguments of the main compactness result. Consequently, the claim is inferred by an application of Zorn's lemma. Finally, having found the coarsest partition we can then show that the corresponding admissible displacement field is uniquely determined up to piecewise infinitesimal rigid motions. 
In Section 6.1 we derive the main $\Gamma$-limit, where the elastic part can be treated as in $[32,42]$ and for the surface energy we separate the effects arising from the segmentation energy and the crack energy inside the components by employing a structure theorem for Caccioppoli partitions (see Theorem 3.5 below). At this point we also establish a result including external loads.

Finally, in Section 6.2 we prove a cleavage law and extend the results obtained in $[29,30,37]$ to the case of uniaxial compression, where we essentially follow the proof in [31,37], in particular using a piecewise rigidity result in $S B D$ (see [15]) and a structure theorem on the boundary of sets of finite perimeter (see [25]). It turns out that in the linearized limit the behavior for compression and extension is virtually identical. We briefly note that to avoid unphysical effects such as self-penetrability, further modeling assumptions would be necessary.

\section{The Model and Main Results}

\subsection{The Nonlinear Model}

Let $\Omega \subset \mathbb{R}^{2}$ open, bounded with Lipschitz boundary. Recall the properties of the space $\operatorname{SBV}\left(\Omega, \mathbb{R}^{2}\right)$, frequently abbreviated as $S B V(\Omega)$ hereafter, in Section 3.1. Fix a (large) constant $M>0$ and define

$$
S B V_{M}(\Omega)=\left\{y \in \operatorname{SBV}\left(\Omega, \mathbb{R}^{2}\right):\|y\|_{\infty}+\|\nabla y\|_{\infty} \leqq M, \mathcal{H}^{1}\left(J_{y}\right)<+\infty\right\} .
$$

Let $W: \mathbb{R}^{2 \times 2} \rightarrow[0, \infty)$ be a frame-indifferent stored energy density with $W(F)=$ 0 iff $F \in S O(2)$. Assume that $W$ is continuous, $C^{3}$ in a neighborhood of $S O(2)$ and scales quadratically at $S O(2)$ in the direction perpendicular to infinitesimal rotations. In other words, there is a positive constant $c$ such that

$$
W(F) \geqq c \operatorname{dist}^{2}(F, S O(2)) \text { for all } F \in \mathbb{R}^{2 \times 2} \text { with }|F| \leqq M .
$$

For $\varepsilon>0$ define the Griffith-energy $E_{\varepsilon}: S B V_{M}(\Omega) \rightarrow[0, \infty)$ by

$$
E_{\varepsilon}(y)=\frac{1}{\varepsilon} \int_{\Omega} W(\nabla y(x)) \mathrm{d} x+\mathcal{H}^{1}\left(J_{y}\right) .
$$

We briefly note that we can also treat inhomogeneous materials where the energy density has the form $W: \Omega \times \mathbb{R}^{2 \times 2} \rightarrow[0, \infty)$. Moreover, it suffices to assume $W \in C^{2, \alpha}$, where $C^{2, \alpha}$ is the Hölder space with exponent $\alpha>0$.

The main goal of the present work is the identification of an effective linearized Griffith energy in the small strain limit which is related to the nonlinear energies $E_{\varepsilon}$ through $\Gamma$-convergence. In this context, we also discuss minimization problems associated to $E_{\varepsilon}$ for given body forces or boundary data. Moreover, we will investigate the limiting model which appears to be more general than many other Griffith functionals in the realm of linearized elasticity (cf. for example $[7,13,14,34,43]$ ) as the limiting configuration not only consists of a displacement field, but also of a coarse partition of the domain and associated rigid motions. In particular, it will turn out that there are various scales for the size of the crack opening occurring in the system. 
Remark 2.1. The threshold $M$ in (2.1) may be chosen arbitrarily large, but is fixed. Confining $y$ in this way effectively models a large box containing the deformed specimen. The restriction on $\nabla y$ is necessary for technical reasons as it allows us to apply a quantitative piecewise rigidity estimate, see Theorem 3.10.

Let us mention that (almost) minimizers of the nonlinear energy $\int_{\Omega \backslash \overline{J_{y}}} W(\nabla y)$ (for given boundary data) are possibly not Lipschitz continuous as particularly at nonsmooth points of the boundary $\partial\left(\Omega \backslash \overline{J_{y}}\right)$ (for example at crack tips) the deformation gradient is expected to form singularities. Consequently, the constraint $\|\nabla y\|_{\infty} \leqq M$ is a real restriction on the class of admissible configurations from a mathematical point of view.

On the other hand, for materials undergoing brittle fracture there is typically a critical strain (and stress), beyond which failure occurs, and therefore the uniform bound on the absolute continuous part of the gradient has a reasonable mechanical interpretation. Moreover, the energy of certain atomistic systems can be related to (2.3) when deformations are identified with piecewise affine interpolations on cells of microscopic size (see for example [9,31]). (Note that in discrete systems the parameter $\varepsilon$ represents not only the order of the elastic energy, but also the typical interatomic distance.) In this context, the bound $\|\nabla y\|_{\infty} \leqq M$ is naturally satisfied. In fact, on cells exceeding such a threshold, also called ultimate strain (see [8]), a discontinuous interpolation with bounded deformation gradient is introduced and their contribution to the energy then enters through the surface part of the energy functional.

Finally, let us mention that particularly from a computational point of view it is interesting to combine a continuum model as (2.3) with an atomistic approach using the quasicontinuum method introduced in [44]. Here the underlying idea is to split the domain into a bulk part with a coarse, continuum description, and into certain critical regions characterized by fast variations of the deformation gradient (such as regions near a dislocation core or a crack tip) where the problem is treated as a fully atomistic system at scale $\varepsilon$ (see [36]).

\subsection{The Segmentation Problem}

As a first natural approach to the problem we concern ourselves with the question of whether the functionals $E_{\varepsilon}$ can be related to a limiting functional for $\varepsilon \rightarrow 0$ in terms of the deformations. We observe that for configurations with uniformly bounded energy $E_{\varepsilon}\left(y_{\varepsilon}\right)$, the absolute continuous part of the gradient satisfies $\nabla y_{\varepsilon} \approx$ $S O(2)$ as the stored energy density is frame-indifferent and minimized on $S O(2)$. Assuming that $y_{\varepsilon} \rightarrow y$ in $L^{1}$, one can show that $\nabla y \in S O(2)$ almost everywhere, applying lower semicontinuity results for $S B V$ functions (see [35]) and using the fact that the quasiconvex envelope of $W$ is minimized exactly on $S O(2)$ (see [45]).

A piecewise rigidity result by Chambolle, Giacomini and Ponsiglione (see Theorem 3.9 below) generalizing the classical Liouville result for smooth functions now states that an $S B V$ function $y$ satisfying the constraint $\nabla y \in S O(2)$ almost everywhere is a collection of an at most countable family of rigid deformations, that is the body may be divided into different components each of which subject to a different rigid motion. 
Consequently, the limit of the sequence $E_{\varepsilon}$ (in the sense of $\Gamma$-convergence) is given by the functional which is finite for piecewise rigid motions and measures the segmentation energy which is necessary to disconnect the body. The exact statement is formulated in Corollary 2.9 as a direct consequence of our main $\Gamma$-convergence result in Theorem 2.7 .

Apparently this simple limiting model does not account for the elastic deformations which are typically present in the nonlinear models. Consequently, to obtain a better understanding of the problem it is desirable to pass to rescaled configurations and to derive a limiting linearized energy as it was performed in [21] in the framework of nonlinear elasticity theory. The main ingredient in that analysis is a quantitative rigidity result due to Friesecke, James and Müller (see Theorem 3.8). The starting point for our analysis will be a corresponding quantitative result in the SBD setting (see [28] or Theorem 3.10) adapted for Griffith functionals of the form (2.3) where both elastic bulk and surface contributions are present.

\subsection{Compactness and Limiting Configurations}

We now present our main compactness result for rescaled displacement fields. As a preparation, recall the notion and basic properties of a Caccioppoli partition in Section 3.2. For a given (ordered) Caccioppoli partition $\mathcal{P}=\left(P_{j}\right)_{j}$ of $\Omega$ let

$$
\mathcal{R}(\mathcal{P})=\left\{T: \Omega \rightarrow \mathbb{R}^{2}: T(x)=\sum_{j} \chi_{P_{j}}(x)\left(R_{j} x+b_{j}\right), R_{j} \in S O(2), b_{j} \in \mathbb{R}^{2}\right\}
$$

be the set of corresponding piecewise rigid motions. Likewise we define the set of piecewise infinitesimal rigid motions, denoted by $\mathcal{A}(\mathcal{P})$, replacing $R_{j} \in S O(2)$ by $A_{j} \in \mathbb{R}_{\text {skew }}^{2 \times 2}=\left\{A \in \mathbb{R}^{2 \times 2}: A=-A^{T}\right\}$. Moreover, we define the triples

$$
\begin{aligned}
\mathcal{D} & :=\{(u, \mathcal{P}, T): u \in \operatorname{SBV}(\Omega), \mathcal{P} \text { C.-partition of } \Omega, T \in \mathcal{R}(\mathcal{P})\}, \\
\mathcal{D}_{\infty} & :=\left\{(u, \mathcal{P}, T): \mathcal{P} \text { C.-partition of } \Omega, T \in \mathcal{R}(\mathcal{P}), \nabla T^{T} u \in G S B D^{2}(\Omega)\right\} .
\end{aligned}
$$

Here $\nabla T$ denotes the absolutely continuous part of $D T$. The space $G S D^{2}(\Omega)$ generalizes the definition of the space $\operatorname{SBD}(\Omega)$ based on certain slicing properties, see Section 3.1. Define $e(G)=\frac{G^{T}+G}{2}$ for all $G \in \mathbb{R}^{2 \times 2}$ and denote by $\partial^{*}$ the essential boundary (see below (3.7)). Let $A \triangle B$ be the symmetric difference of two sets $A, B \subset \mathbb{R}^{2}$. We now formulate the main compactness theorem.

Theorem 2.2. Let $\Omega \subset \mathbb{R}^{2}$ open, bounded with Lipschitz boundary. Let $M>0$ and $\varepsilon_{k} \rightarrow 0$ as $k \rightarrow \infty$. If $E_{\varepsilon_{k}}\left(y_{k}\right) \leqq C$ for a sequence $\left(y_{k}\right)_{k} \subset S B V_{M}(\Omega)$, then there exists a subsequence (not relabeled) such that the following holds: There are triples $\left(u_{k}, \mathcal{P}_{k}, T_{k}\right) \in \mathcal{D}$, where $\mathcal{P}_{k}=\left(P_{j}^{k}\right)_{j}$, and $c>0$ with

(i) $u_{k}(x)-\varepsilon_{k}^{-1 / 2}\left(y_{k}(x)-T_{k}(x)\right) \rightarrow 0$ for almost every $x \in \Omega$ for $k \rightarrow \infty$,

(ii) $\left\|\nabla u_{k}\right\|_{L^{\infty}(\Omega)} \leqq c \varepsilon_{k}^{-1 / 8}$ for $k \in \mathbb{N}$ 
such that we find a limiting triple $(u, \mathcal{P}, T) \in \mathcal{D}_{\infty}$ with

$$
\begin{aligned}
& \text { (i) }\left|P_{j}^{k} \triangle P_{j}\right| \rightarrow 0 \text { for all } j \in \mathbb{N}, \\
& \text { (ii) } T_{k} \rightarrow T \text { in } L^{2}\left(\Omega, \mathbb{R}^{2}\right), \quad \nabla T_{k} \rightarrow \nabla T \text { in } L^{2}\left(\Omega, \mathbb{R}^{2 \times 2}\right)
\end{aligned}
$$

for $k \rightarrow \infty$ and

(i) $u_{k} \rightarrow u$ almost everywhere in $\Omega$,

(ii) $e\left(\nabla T_{k}^{T} \nabla u_{k}\right) \rightarrow e\left(\nabla T^{T} \nabla u\right)$ weakly in $L^{2}\left(\Omega, \mathbb{R}_{\mathrm{sym}}^{2 \times 2}\right)$

for $k \rightarrow \infty$. Moreover, for the elastic and crack energy we obtain

$$
\begin{aligned}
& \text { (i) } \frac{1}{\varepsilon_{k}} \int_{\Omega} W\left(\nabla y_{k}\right)+o(1) \geqq \frac{1}{\varepsilon_{k}} \int_{\Omega} W\left(\mathbf{I d}+\sqrt{\varepsilon_{k}} \nabla T_{k}^{T} \nabla u_{k}\right) \text { as } k \rightarrow \infty, \\
& \text { (ii) } \liminf _{k \rightarrow \infty} \mathcal{H}^{1}\left(J_{y_{k}}\right) \geqq \mathcal{H}^{1}\left(\bigcup_{j} \partial^{*} P_{j} \cap \Omega\right)+\mathcal{H}^{1}\left(J_{u} \backslash \bigcup_{j} \partial^{*} P_{j}\right) .
\end{aligned}
$$

Recall that the central object in linearized elasticity is the symmetric part of the gradient, which comes from the facts that: (1) deformations are linearized around the identity; and (2) the orthogonal space to $S O(d)$ at the identity is given by the symmetric matrices (see for example [21]). In the present context, where we possibly linearize around different rigid motions, the symmetrized gradient is accordingly replaced by $e\left(\nabla T^{T} u\right)$ in both the limiting description and the convergence (see (2.5) and (2.8)(ii), respectively).

In (2.7) and (2.8) the convergence for the partitions, rigid motions and displacement fields is given, respectively. Moreover, (2.6) and (2.9) represent compatibility conditions for the triple $\left(u_{k}, \mathcal{P}_{k}, T_{k}\right)$. In general, $u_{k}$ is a modification of the rescaled displacement $\varepsilon_{k}^{-1 / 2}\left(y_{k}-T_{k}\right)$, but asymptotically both configurations coincide (see (2.6)(i)). Moreover, the modifications can be constructed such that $\nabla u_{k}$ is suitably controllable. (The exponent $-\frac{1}{8}$ is chosen for definiteness only and could be replaced by any small negative exponent, cf. (3.10)(iv) and the paragraph below Theorem 3.10.) Finally, the elastic and crack energy associated to the triples are controlled by the corresponding energies of $y_{k}$ up to small errors vanishing in the limit (see (2.9)).

Definition 2.3. We say a sequence $\left(y_{k}\right)_{k} \subset S B V_{M}(\Omega)$ is asymptotically represented by a limiting triple $(u, \mathcal{P}, T) \in \mathcal{D}_{\infty}$, and write $y_{k} \rightarrow(u, \mathcal{P}, T)$, if there is a sequence of triples $\left(u_{k}, \mathcal{P}_{k}, T_{k}\right) \in \mathcal{D}$ such that (2.6)-(2.9) are satisfied.

Although we use the notation $\rightarrow$ and call $(u, \mathcal{P}, T)$ a limiting triple, it is clear that Definition 2.3 cannot be understood as a convergence in the usual sense. In particular, in the small strain limit a tripling of the variables occurs, which is a specific feature of our limiting model. Additionally, the triples $(u, \mathcal{P}, T)$ given by the main compactness theorem for a sequence $\left(y_{k}\right)_{k}$ are not determined uniquely, but crucially depend on the choice of the sequences $\left(\mathcal{P}_{k}\right)_{k}$ and $\left(T_{k}\right)_{k}$. To illustrate the latter phenomenon, we consider the following example: 
Example 2.4. Consider $\Omega=(0,3) \times(0,1), \Omega_{1}=(0,1) \times(0,1), \Omega_{2}=(1,2) \times$ $(0,1), \Omega_{3}=(2,3) \times(0,1)$ and

$$
y_{k}=\mathbf{i d} \chi_{\Omega_{1}}+\left(\mathbf{i d}+\alpha \sqrt{\varepsilon_{k}}\right) \chi_{\Omega_{2}}+\left(\mathbf{i d}+\varepsilon_{k}^{1 / 4}\right) \chi_{\Omega_{3}}
$$

for $\alpha \in \mathbb{R}^{2}$. Then for $b \in \mathbb{R}^{2}$, possible alternatives are, for example,

(1) $P_{1}^{1}=\Omega_{1}, P_{2}^{1}=\Omega_{2}, P_{3}^{1}=\Omega_{3}$ with $T_{k}^{1}=y_{k}$ on $\Omega$,

(2) $P_{1}^{2}=\Omega_{1} \cup \Omega_{2}, P_{2}^{2}=\Omega_{3}$ with $T_{k}^{2}=\mathbf{i d} \chi_{\Omega_{1} \cup \Omega_{2}}+\left(\mathbf{i d}+\varepsilon_{k}{ }^{1 / 4}-b \sqrt{\varepsilon_{k}}\right) \chi_{\Omega_{3}}$.

Letting $u_{k}^{i}=\varepsilon_{k}{ }^{-\frac{1}{2}}\left(y_{k}-T_{k}^{i}\right)$ for $i=1,2$ we obtain in the limit $\varepsilon_{k} \rightarrow 0$ the unique rigid motion $T=$ id and the different configurations

$$
\begin{array}{ll}
\text { (1) } u^{1}=0, & P_{1}^{1}=\Omega_{1}, P_{2}^{1}=\Omega_{2}, P_{3}^{1}=\Omega_{3}, \\
\text { (2) } u^{2}=0 \cdot \chi_{\Omega_{1}}+\alpha \chi_{\Omega_{2}}+b \chi_{\Omega_{3}}, & P_{1}^{2}=\Omega_{1} \cup \Omega_{2}, P_{2}^{2}=\Omega_{3} .
\end{array}
$$

We now introduce a special subclass of partitions in which uniqueness will be guaranteed. The above example already shows that different partitions are not equivalent in the sense that they may contain a different 'amount of information'. Note that on the various elements of the partition the configuration $u$ is defined separately and the different pieces of the domain are not 'aware of each other'. In particular, the difference of the traces of $u$ on $\partial^{*} P_{i} \cap \partial^{*} P_{j}, i \neq j$, does not have any physically reasonable interpretation. On the contrary, in example (2) where we did not split up $\Omega_{1} \cup \Omega_{2}$, we gain the jump height on $\partial \Omega_{1} \cap \partial \Omega_{2}$ as an additional information. The observation that coarser partitions provide more information about the behavior at the jump set motivates the definition of the coarsest partition.

Definition 2.5. Let $\left(y_{k}\right)_{k}$ be a given (sub-)sequence as in Theorem 2.2. Then:

(i) We say a partition $\mathcal{P}$ of $\Omega$ is admissible for $\left(y_{k}\right)_{k}$, and write $\mathcal{P} \in \mathcal{Z}_{P}\left(\left(y_{k}\right)_{k}\right)$, if there exist $u, T$ such that $(u, \mathcal{P}, T) \in \mathcal{D}_{\infty}$ and $y_{k} \rightarrow(u, \mathcal{P}, T)$;

(ii) We say a piecewise rigid motion $T$ is admissible for $\left(y_{k}\right)_{k}$, and write $T \in$ $\mathcal{Z}_{T}\left(\left(y_{k}\right)_{k}\right)$, if there exist $u, \mathcal{P}$ such that $(u, \mathcal{P}, T) \in \mathcal{D}_{\infty}$ and $y_{k} \rightarrow(u, \mathcal{P}, T)$;

(iii) We say a configuration $u$ is admissible for $\left(y_{k}\right)_{k}$ and $\mathcal{P}$, and write $u \in$ $\mathcal{Z}_{u}\left(\left(y_{k}\right)_{k}, \mathcal{P}\right)$, if there exists $T$ such that $(u, \mathcal{P}, T) \in \mathcal{D}_{\infty}$ and $y_{k} \rightarrow(u, \mathcal{P}, T)$;

(iv) We say a partition $\mathcal{P}$ of $\Omega$ is a coarsest partition for $\left(y_{k}\right)_{k}$ if the following holds: The partition is admissible, that is $\mathcal{P} \in \mathcal{Z}_{P}\left(\left(y_{k}\right)_{k}\right)$. Moreover, for all admissible $u \in \mathcal{Z}_{u}\left(\left(y_{k}\right)_{k}, \mathcal{P}\right)$ and all corresponding triples $\left(u_{k}, \mathcal{P}_{k}, T_{k}\right) \in \mathcal{D}$ satisfying (2.6)-(2.9) the mappings $T_{k}=\sum_{j}\left(R_{j}^{k} \cdot+b_{j}^{k}\right) \chi_{P_{j}^{k}}$ fulfill

$$
\frac{\left|R_{i}^{k}-R_{j}^{k}\right|+\left|b_{i}^{k}-b_{j}^{k}\right|}{\sqrt{\varepsilon_{k}}} \rightarrow \infty
$$

for all $i, j \in \mathbb{N}, i \neq j$ and $k \rightarrow \infty$.

In Lemma 5.2 below we find an equivalent characterization of coarsest partitions being the maximal elements of the partial order on the sets of admissible partitions which is induced by subordination. Loosely speaking, the above definition particularly implies that given a coarsest partition a region of the domain is 
partitioned into different sets $\left(P_{j}\right)_{j}$ if and only if the (scaled) jump height $\varepsilon_{k}^{-1 / 2}\left[y_{k}\right]$ on $\left(\partial^{*} P_{j}\right)_{j}$ tends to infinity (cf. Example 2.4 ).

Recall the definition of the piecewise infinitesimal rigid motions $\mathcal{A}(\mathcal{P})$ below (2.4). We now obtain a unique characterization of the limiting configuration up to piecewise infinitesimal rigid motions.

Theorem 2.6. Let $\varepsilon_{k} \rightarrow 0$ be given. Let $\left(y_{k}\right)_{k} \subset S B V_{M}(\Omega)$ be a sequence for which the assertion of Theorem 2.2 holds. Then we have the following:

(i) There is a unique $T \in \mathcal{Z}_{T}\left(\left(y_{k}\right)_{k}\right)$;

(ii) There is a unique coarsest partition $\overline{\mathcal{P}}$ of $\Omega$;

(iii) Given some $u \in \mathcal{Z}_{u}\left(\left(y_{k}\right)_{k}, \overline{\mathcal{P}}\right)$ all admissible limiting configurations are of the form $u+\nabla T \mathcal{A}(\overline{\mathcal{P}})$, that is the limiting configuration is determined uniquely up to piecewise infinitesimal rigid motions.

Going back to Example 2.4, we observe that $T=$ id is uniquely given and that the partition in (2) is the coarsest partition. The non-uniqueness in (iii) is a consequence of the fact that the nonlinear energy is invariant under rigid motions (see also (2) in Example 2.4 for $b \in \mathbb{R}^{2}$ ).

\subsection{The Limiting Linearized Model and $\Gamma$-Convergence}

We now introduce the limiting linearized model, discuss its properties and show that it can be identified as the $\Gamma$-limit of the nonlinear energies $E_{\varepsilon}$. Let $Q=D^{2} W(\mathbf{I d})$ be the Hessian of the stored energy density $W$ at the identity. Define $E: \mathcal{D}_{\infty} \rightarrow[0, \infty)$ by

$$
E(u, \mathcal{P}, T)=\int_{\Omega} \frac{1}{2} Q\left(e\left(\nabla T^{T} \nabla u\right)\right)+\mathcal{H}^{1}\left(J_{u} \backslash \bigcup_{j} \partial^{*} P_{j}\right)+\mathcal{H}^{1}\left(\bigcup_{j} \partial^{*} P_{j} \cap \Omega\right),
$$

where as before $\mathcal{P}=\left(P_{j}\right)_{j}$. Recall that a triple of the limiting model consists of a partition, a corresponding piecewise rigid motion and a displacement field. We emphasize that in contrast to the nonlinear model (see (2.1) and Remark 2.1), there are no restrictive bounds on the functions $u$ and their derivatives .

The surface energy of $E$ has two parts. In a fashion similar to Section 2.2, on the right we have the segmentation energy which is necessary to disconnect the components of the body. Moreover, on the left we have the inner crack energy associated to the discontinuity set of the displacement field in each part of the material (see also Remark 2.8(ii) below). Whereas the first two terms of the functional typically appear in the study of linearized Griffith energies, the segmentation energy is a characteristic feature of our general model where the analysis is not restricted to a linearization around a fixed rigid motion.

We now present our main $\Gamma$-convergence result. Recall Definition 2.3.

Theorem 2.7. Let $\Omega \subset \mathbb{R}^{2}$ open, bounded with Lipschitz boundary. Let $M>0$ and $\varepsilon_{k} \rightarrow 0$. Then $E_{\varepsilon_{k}}$ converges to $E$ in the sense of $\Gamma$-convergence, that is: 
(i) $\Gamma-\lim$ inf inequality: for all $(u, \mathcal{P}, T) \in \mathcal{D}_{\infty}$ and for all sequences $\left(y_{k}\right)_{k} \subset$ $S B V_{M}(\Omega)$ with $y_{k} \rightarrow(u, \mathcal{P}, T)$ we have

$$
\liminf _{k \rightarrow \infty} E_{\varepsilon_{k}}\left(y_{k}\right) \geqq E(u, \mathcal{P}, T)
$$

(ii) Existence of recovery sequences: for every $(u, \mathcal{P}, T) \in \mathcal{D}_{\infty}$ with $u \in L^{2}(\Omega)$ we find a sequence $\left(y_{k}\right)_{k} \subset S B V_{M}(\Omega)$ such that $y_{k} \rightarrow(u, \mathcal{P}, T)$ and

$$
\lim _{k \rightarrow \infty} E_{\varepsilon_{k}}\left(y_{k}\right)=E(u, \mathcal{P}, T) .
$$

Remark 2.8. (i) The limiting model could equivalently be formulated with $v=$ $\nabla T^{T} u$ in place of the displacement field $u$. (Accordingly, replace $u_{k}$ by $v_{k}=$ $\nabla T_{k}^{T} u_{k}$ in Theorem 2.2.) This alternative notation simplifies the description of the elastic energy in (2.11), but does not account for the fact that the linearization was possibly performed around different rigid motions.

(ii) Using the local structure of Caccioppoli partitions (see Theorem 3.5 and recall (3.7)) the limiting energy can equivalently be written as

$$
\sum_{j}\left(\int_{P_{j}} \frac{1}{2} Q\left(e\left(R_{j}^{T} \nabla u\right)\right)+\mathcal{H}^{1}\left(J_{u} \cap\left(P_{j}\right)^{1}\right)+\frac{1}{2} \mathcal{H}^{1}\left(\partial^{*} P_{j} \cap \Omega\right)\right) .
$$

(iii) For configurations $(u, \overline{\mathcal{P}}, T)$ defined in terms of the coarsest partition $\overline{\mathcal{P}}$ there is an additional interpretation for the crack opening of the sequence of deformations $y_{\varepsilon}$ : (1) The jumps on $\bigcup_{j} \partial^{*} P_{j}$ are associated to jump heights $\gg \sqrt{\varepsilon}$ and (2) the jump heights corresponding to the inner crack energy are of the order $\sqrt{\varepsilon}$, which illustrates the multiscale nature of the model. In fact, (1) follows from (2.10) and (2) is a consequence of (2.6)(i).

(iv) On a component $P_{j}$ of $\overline{\mathcal{P}}$ the body may still be disconnected by the jump set $\left(P_{j}\right)^{1} \cap J_{u}$ forming a finer partition of the specimen. However, in contrast to the boundary of $\overline{\mathcal{P}}$ the jump heights have a meaningful physical interpretation.

(v) In general, the partition induced by the macroscopic jumps (represented by $\left.J_{T}\right)$ is coarser than $\overline{\mathcal{P}}$, that is $\mathcal{H}^{1}\left(\bigcup_{j} \partial^{*} P_{j} \backslash\left(\partial \Omega \cup J_{T}\right)\right)>0$, cf. Example 2.4.

As a direct consequence of Theorem 2.7 we get that the $\Gamma$-limit of the same functionals $E_{\varepsilon_{k}}$ with respect to the much weaker notion of $L^{1}$-convergence of the unrescaled deformations $y_{k}$ is given by the segmentation energy.

Corollary 2.9. Let $\Omega \subset \mathbb{R}^{2}$ open, bounded with Lipschitz, boundary. Let $M>0$ and $\varepsilon_{k} \rightarrow 0$. Then $E_{\varepsilon_{k}} \Gamma$-converge to $E_{\text {seg }}$ with respect to the $L^{1}(\Omega)$-convergence, where

$$
E_{\mathrm{seg}}(y)= \begin{cases}\mathcal{H}^{1}\left(J_{T}\right) & y=T \in \mathcal{R}(\mathcal{P}) \text { for a Caccioppoli partition } \mathcal{P} \\ +\infty & \text { else. }\end{cases}
$$

Note that the segmentation energy in Corollary 2.9 differs from the one in (2.11), see Remark 2.8(v). 


\subsection{Application: External Loads}

For the investigation of minimization problems associated to $E_{\varepsilon}$ it is interesting to take external loads into account. In the context of brittle materials, however, the incorporation of body forces is a delicate problem. Indeed, assumptions on the class of admissible loads have to ensure that no part of the body is broken apart and sent to infinity, which clearly excludes the case of a constant body force (see [19, Remark 3.1]). To avoid the occurrence of such phenomena, it is natural to assume a uniform $L^{\infty}$ bound on the admissible functions, see for example [20,22]. Unfortunately, this is not expedient in our setting since the bound $\|y\|_{\infty} \leqq M$ is futile after passage to rescaled configurations in Theorem 2.2.

Let us mention that in [19] body forces were indispensable to ensure reasonable compactness properties for sequences and to guarantee existence of minimizers. In our setting, however, due to the subtraction of suitable rigid motions on a partition of the domain (cf. (2.6)(i)) we obtain a compactness result without the necessity of additional loading terms.

We fix a sequence $\varepsilon_{k} \rightarrow 0$ and consider the following prototype problem $F_{\varepsilon_{k}}: S B V_{M}(\Omega) \rightarrow[0, \infty)$ with

$$
F_{\varepsilon_{k}}(y)=E_{\varepsilon_{k}}(y)+\frac{\lambda}{\varepsilon_{k}}\left\|y-f_{k}\right\|_{L^{2}(\Omega)}^{2},
$$

where $\lambda>0$ and $\left(f_{k}\right)_{k} \subset S B V_{M}(\Omega)$ a sequence with $\sup _{k} E_{\varepsilon_{k}}\left(f_{k}\right)<\infty$. An expansion yields the constant $\lambda \varepsilon_{k}^{-1} \int_{\Omega}\left|f_{k}\right|^{2}$, the external load $-2 \lambda \varepsilon_{k}^{-1} \int_{\Omega} f_{k} \cdot y$ and the term $\lambda \varepsilon_{k}^{-1} \int_{\Omega}|y|^{2}$. The latter can be interpreted as an artificial confining potential, which prevents parts of the body from being sent to infinity.

We assume that there is a triple $\left(g, \mathcal{P}_{g}, T_{g}\right) \in \mathcal{D}_{\infty}$ such that $f_{k} \rightarrow\left(g, \mathcal{P}_{g}, T_{g}\right)$ in the sense of Definition 2.3 and that for the associated triples $\left(g_{k}, \mathcal{P}_{k}^{g}, T_{k}^{g}\right) \in \mathcal{D}$ satisfying (2.6)-(2.9) we have $\varepsilon_{k}^{-1 / 2}\left(f_{k}-T_{k}^{g}\right) \rightarrow g$ in $L^{2}(\Omega)$ and $\mathcal{P}_{k}^{g}=\mathcal{P}_{g}$ for all $k \in \mathbb{N}$. (Note that up to a subsequence the convergence in the sense of Definition 2.3 is already guaranteed by Theorem 2.2.) Moreover, we suppose that $\mathcal{P}_{g}$ is the coarsest partition given by Theorem 2.6(ii) and write $\mathcal{P}_{g}=\left(P_{j}^{g}\right)_{j}$. By $\mathcal{C}_{g} \subset \mathcal{D}_{\infty}$ we denote the set of triples $(u, \mathcal{P}, T) \in \mathcal{D}_{\infty}$ with $T=T_{g}$ and the property that $\mathcal{P}_{g}$ is coarser than $\mathcal{P}$, that is for each $P_{j}$ there exists $P_{i}^{g}$ with $\left|P_{j} \backslash P_{i}^{g}\right|=0$.

Lemma 2.10. Let $\left(y_{k}\right)_{k} \subset S B V_{M}(\Omega)$ be a sequence with $F_{\varepsilon_{k}}\left(y_{k}\right) \leqq C$ and $y_{k} \rightarrow$ $(u, \mathcal{P}, T) \in \mathcal{D}_{\infty}$ in the sense of Definition 2.3. Then $(u, \mathcal{P}, T) \in \mathcal{C}_{g}$.

Recalling (2.11) we introduce the limiting energy $F_{g}: \mathcal{D}_{\infty} \rightarrow[0, \infty]$ by $F_{g}(u, \mathcal{P}, T)= \begin{cases}E(u, \mathcal{P}, T)+\min _{v \in u+\nabla T \mathcal{A}(\mathcal{P})} \lambda\|v-g\|_{L^{2}(\Omega)}^{2} & \text { if }(u, \mathcal{P}, T) \in \mathcal{C}_{g}, \\ +\infty & \text { else, }\end{cases}$

where $\mathcal{A}(\mathcal{P})$ as defined below (2.4). Similar to the functional in (2.11), $F_{g}$ is invariant under infinitesimal rigid motions on the components of the partition $\mathcal{P}$. However, the additional term on the right induces a symmetry breaking and there is exactly one distinguished configuration $u^{*}$ in the class $u+\nabla T \mathcal{A}(\mathcal{P})$ (cf. (iii) in Theorem 2.6) which satisfies $\min _{v \in u+\nabla T \mathcal{A}(\mathcal{P})}\|v-g\|_{L^{2}(\Omega)}^{2}=\left\|u^{*}-g\right\|_{L^{2}(\Omega)}^{2}$. We close this section with a corresponding $\Gamma$-convergence result. 
Theorem 2.11. Let $\Omega \subset \mathbb{R}^{2}$ open, bounded with Lipschitz boundary. Let $M>0$, $\varepsilon_{k} \rightarrow 0$ and $\left(f_{k}\right)_{k}, g$ as above. Then $F_{\varepsilon_{k}}$ converges to $F_{g}$ in the sense of $\Gamma$ convergence. (Replace $E_{\varepsilon_{k}}$ by $F_{\varepsilon_{k}}$ and $E$ by $F_{g}$ in (i),(ii) of Theorem 2.7.) Moreover, we have

$$
\lim _{k \rightarrow \infty} \inf _{y \in S B V_{M}(\Omega)} F_{\varepsilon_{k}}(y)=\min _{(u, \mathcal{P}, T) \in \mathcal{D}_{\infty}} F_{g}(u, \mathcal{P}, T),
$$

and (almost) minimzers of $F_{\varepsilon_{k}}$ converge (up to subsequences) to minimizers of $F_{g}$ in the sense of Definition 2.3.

\subsection{Application: Cleavage Laws}

In fracture mechanics it is a major challenge to identify critical loads at which a body fails and to determine the geometry of crack paths that occur in the fractured regime. As another application of the above results we now finally derive such a cleavage law. We consider a special boundary value problem of uniaxial compression/extension. Let $\Omega=(0, l) \times(0,1), \Omega^{\prime}=(-\eta, l+\eta) \times(0,1)$ for $l>0, \eta>0$ and for $a_{\varepsilon} \in \mathbb{R}$ define

$$
\mathcal{A}\left(a_{\varepsilon}\right):=\left\{y \in S B V_{M}\left(\Omega^{\prime}\right): y_{1}(x)=\left(1+a_{\varepsilon}\right) x_{1} \text { for } x_{1} \leqq 0 \text { or } x_{1} \geqq l\right\} .
$$

As usual in the theory of $S B V$ functions the boundary values have to be imposed in small neighborhoods of the boundary. In what follows, the elastic part of the energy (2.3) still only depends on $\left.y\right|_{\Omega}$, whereas the surface energy is given by $\mathcal{H}^{1}\left(J_{y}\right)$ with $J_{y} \subset \Omega^{\prime}$. In particular, jumps on $\{0, l\} \times(0,1)$ contribute to the energy $E_{\varepsilon}(y)$. (Also compare a similar discussion before [31, Theorem 2.2].) The present problem in the framework of continuum fracture mechanics with isotropic surface energies is a slightly simplified model of the problem considered in [29,31].

As a preparation, define $\alpha$ such that $\inf \left\{Q(F): \mathbf{e}_{1}^{T} F \mathbf{e}_{1}=1\right\}=\alpha$ and observe $\inf \left\{Q(F): \mathbf{e}_{1}^{T} F \mathbf{e}_{1}=a\right\}=\alpha a^{2}$ for all $a \in \mathbb{R}$. Moreover, let $F^{a} \in \mathbb{R}_{\text {sym }}^{2 \times 2}$ be the unique matrix such that $\mathbf{e}_{1}^{T} F^{a} \mathbf{e}_{1}=a$ and $Q\left(F^{a}\right)=\inf \left\{Q(F): \mathbf{e}_{1}^{T} F \mathbf{e}_{1}=a\right\}=$ $\alpha a^{2}$.

We recall that the proof of the cleavage laws in [29,30,37] fundamentally relied on the application of certain slicing techniques which were not suitable to treat the case of compression. Having general compactness and $\Gamma$-convergence results we can now complete the picture about cleavage laws by extending the results to the case of uniaxial compression.

Theorem 2.12. Suppose that $a_{\varepsilon} / \sqrt{\varepsilon} \rightarrow a \in[-\infty, \infty]$. The limiting minimal energy is given by

$$
\lim _{\varepsilon \rightarrow 0} \inf \left\{E_{\varepsilon}(y): y \in \mathcal{A}\left(a_{\varepsilon}\right)\right\}=\min \left\{\frac{1}{2} \alpha l a^{2}, 1\right\} .
$$

Let $a_{\text {crit }}:=\sqrt{\frac{2 \alpha}{l}}$. For every sequence $\left(y_{\varepsilon}\right)_{\varepsilon}$ of almost minimizers, up to passing to subsequences, we get $\varepsilon^{-1 / 2}\left(y_{\varepsilon}(x)-x\right) \rightarrow u(x)$ for almost every $x \in \Omega$, where 
(i) if $|a|<a_{\text {crit }}, u(x)=(0, s)+F^{a} x$ for $s \in \mathbb{R}$, and

(ii) if $|a|>a_{\text {crit }}, u(x)=\left\{\begin{array}{ll}(0, s) & x_{1}<p, \\ (l a, t) & x_{1}>p,\end{array}\right.$ for $s, t \in \mathbb{R}, p \in(0, l)$.

Let us emphasize that the cleavage law is derived for a special geometry of $\Omega$ by solving a static, global minimization problem similarly as in [10,30,37]. An accurate prediction of crack propagation under tensile loading is beyond the scope of the present contribution.

\section{Preliminaries}

In this section we collect the definitions as well as basic properties of $S B V$ and $S B D$ functions and state the rigidity estimates which are necessary for the derivation of our main compactness result.

\section{1. (G)SBV and $(G) S B D$ Functions}

Let $\Omega \subset \mathbb{R}^{d}$ open, bounded with Lipschitz boundary. Recall that the space $\operatorname{SBV}\left(\Omega, \mathbb{R}^{d}\right)$, abbreviated as $\operatorname{SBV}(\Omega)$ hereafter, of special functions of bounded variation consists of functions $y \in L^{1}\left(\Omega, \mathbb{R}^{d}\right)$ whose distributional derivative $D y$ is a finite Radon measure, which splits into an absolutely continuous part with density $\nabla y$ with respect to Lebesgue measure and a singular part $D^{s} y$ whose Cantor part vanishes and thus is of the form

$$
D^{s} y=[y] \otimes v_{y} \mathcal{H}^{d-1}\left\lfloor J_{y} .\right.
$$

Here $\mathcal{H}^{d-1}$ denotes the $(d-1)$-dimensional Hausdorff measure, $J_{y}$ (the 'crack path') is an $\mathcal{H}^{d-1}$-rectifiable set in $\Omega, v_{y}$ is a normal of $J_{y}$ and $[y]=y^{+}-y^{-}$ (the 'crack opening') with $y^{ \pm}$being the one-sided limits of $y$ at $J_{y}$. If in addition $\nabla y \in L^{2}(\Omega)$ and $\mathcal{H}^{d-1}\left(J_{y}\right)<\infty$, we write $y \in S B V^{2}(\Omega)$. See [3] for the basic properties of this function space.

Likewise, we say that a function $y \in L^{1}\left(\Omega, \mathbb{R}^{d}\right)$ is a special function of bounded deformation if the symmetrized distributional derivative $E y:=\frac{(D y)^{T}+D y}{2}$ is a finite $\mathbb{R}_{\text {sym }}^{d \times d}$-valued Radon measure with vanishing Cantor part. It can be decomposed as

$$
E y=e(\nabla y) \mathcal{L}^{d}+E^{s} y=e(\nabla y) \mathcal{L}^{d}+\left.[y] \odot \nu_{y} \mathcal{H}^{d-1}\right|_{J_{y}},
$$

where $e(\nabla y)$ is the absolutely continuous part of $E y$ with respect to the Lebesgue measure $\mathcal{L}^{d},[y], v_{y}, J_{y}$ as before and $a \odot b=\frac{1}{2}(a \otimes b+b \otimes a)$. For basic properties of this function space we refer to $[2,4]$.

To treat variational problems as considered in Section 2 (see in particular (2.3)) the spaces $S B V(\Omega)$ and $\operatorname{SBD}(\Omega)$ are not adequate due to the lacking $L^{\infty}$-bound being essential in the compactness theorems. To overcome this difficulty the space of $G S B V(\Omega)$ was introduced consisting of all $\mathcal{L}^{d}$-measurable functions $y: \Omega \rightarrow \mathbb{R}^{d}$ such that for every $\phi \in C^{1}\left(\mathbb{R}^{d}\right)$ with the support of $\nabla \phi$ compact, the composition $\phi \circ y$ belongs to $S B V_{\text {loc }}(\Omega)$ (see [23]). In this new setting one may obtain a more 
general compactness result (see [3, Theorem 4.36]). Unfortunately, this approach cannot be pursued in the framework of $S B D$ functions as for a function $y \in S B D(\Omega)$ the composition $\phi \circ y$ typically does not lie in $S B D(\Omega)$. In [18], Dal Maso suggested another approach which is based on certain properties of one-dimensional slices.

First we have to introduce some notation. For every $\xi \in \mathbb{R}^{d} \backslash\{0\}$, for every $s \in \mathbb{R}^{d}$ and for every $B \subset \Omega$ we let

$$
B^{\xi, s}=\{t \in \mathbb{R}: s+t \xi \in B\} .
$$

Furthermore, define the hyperplane $\Pi^{\xi}=\left\{x \in \mathbb{R}^{d}: x \cdot \xi=0\right\}$. Moreover, for every function $y: B \rightarrow \mathbb{R}^{d}$ we introduce the function $y^{\xi, s}: B^{\xi, s} \rightarrow \mathbb{R}^{d}$ by

$$
y^{\xi, s}(t)=y(s+t \xi)
$$

and $\hat{y}^{\xi, s}: B^{\xi, s} \rightarrow \mathbb{R}$ by $\hat{y}^{\xi, s}(t)=y(s+t \xi) \cdot \xi$. If $\hat{y}^{\xi, s} \in S B V\left(B^{\xi, s}, \mathbb{R}\right)$ and $J_{\hat{y} \xi, s}$ denotes the approximate jump set, we define

$$
J_{\hat{y} \xi, s}^{1}:=\left\{t \in J_{\hat{y} \xi, s}:\left|\left[\hat{y}^{\xi, s}\right](t)\right| \geqq 1\right\} .
$$

The space $G \operatorname{SBD}\left(\Omega, \mathbb{R}^{d}\right)$ of generalized functions of bounded deformation is the space of all $\mathcal{L}^{d}$-measurable functions $y: \Omega \rightarrow \mathbb{R}^{d}$ with the following property: There exists a nonnegative bounded Radon measure $\lambda$ on $\Omega$ such that for all $\xi \in$ $S^{d-1}:=\left\{x \in \mathbb{R}^{d}:|x|=1\right\}$ we have that for $\mathcal{H}^{d-1}$-almost every $s \in \Pi^{\xi}$ the function $\hat{y}^{\xi, s}=y^{\xi, s} \cdot \xi$ belongs to $S B V_{\text {loc }}\left(\Omega^{\xi, s}\right)$ and

$$
\int_{\Pi^{\xi}}\left(\left|D \hat{y}^{\xi, s}\right|\left(B^{\xi, s} \backslash J_{\hat{y} \xi, s}^{1}\right)+\mathcal{H}^{0}\left(B^{\xi, s} \cap J_{\hat{y} \xi, s}^{1}\right)\right) \mathrm{d} \mathcal{H}^{d-1}(s) \leqq \lambda(B)
$$

for all Borel sets $B \subset \Omega$.

We refer to [18] for basic properties of this space. In particular, for later reference we now recall fundamental slicing, compactness and approximation results. We first briefly state the main slicing properties of GSBD functions (see [18, Section 8,9].) Recall definitions (3.2) and (3.3) and let $J_{y}^{\xi}=\left\{x \in J_{y}:[y](x) \cdot \xi \neq 0\right\}$.

Theorem 3.1. Let $y \in G S B D(\Omega)$. For all $\xi \in S^{d-1}$ and $\mathcal{H}^{d-1}$-almost every $s$ in $\Pi^{\xi}=\{x: x \cdot \xi=0\}$ we have $J_{\hat{y} \xi, s}=\left(J_{y}^{\xi}\right)^{\xi, s}$ and

$$
\int_{\Pi^{\xi}} \# J_{\hat{y} \xi, s} d \mathcal{H}^{d-1}(s)=\int_{J_{y}^{\xi}}\left|v_{y} \cdot \xi\right| d \mathcal{H}^{d-1} .
$$

Moreover, the approximate symmetrized gradient $e(\nabla y)$ exists in the sense of $[18$, (9.1)], satisfies $e(\nabla y) \in L^{1}\left(\Omega, \mathbb{R}_{\mathrm{sym}}^{d \times d}\right)$ and for all $\xi \in S^{d-1}$ and $\mathcal{H}^{d-1}$-almost every s in $\Pi^{\xi}$ we have

$$
\xi^{T} e(\nabla y(s+t \xi)) \xi=\left(\hat{y}^{\xi, s}\right)^{\prime}(t) \text { for almost every } t \in \Omega^{\xi, s} .
$$

If, in addition, $e(\nabla y) \in L^{2}(\Omega)$ and $\mathcal{H}^{d-1}\left(J_{y}\right)<\infty$, we write $y \in G S B D^{2}(\Omega)$. Similar properties for $S B V$ functions may be found in [3, Section 3.11]. We now state a general compactness result in GSBD proved in [18, Theorem 11.3] which we slightly adapt for our purposes. 
Theorem 3.2. Let $\left(y_{k}\right)_{k}$ be a sequence in $G S B D^{2}(\Omega)$. Suppose that there exist a constant $M>0$ and an increasing continuous functions $\psi:[0, \infty) \rightarrow[0, \infty)$ with $\lim _{t \rightarrow \infty} \psi(t)=+\infty$ such that

$$
\int_{\Omega} \psi\left(\left|y_{k}\right|\right)+\int_{\Omega}\left|e\left(\nabla y_{k}\right)\right|^{2}+\mathcal{H}^{d-1}\left(J_{y_{k}}\right) \leqq M
$$

for every $k \in \mathbb{N}$. Then there exist a subsequence, still denoted by $\left(y_{k}\right)_{k}$, and a function $y \in G \operatorname{SBD}^{2}(\Omega)$ such that

$$
\begin{aligned}
& y_{k} \rightarrow y \quad \text { pointwise almost everywhere in } \Omega, \\
& e\left(\nabla y_{k}\right) \rightarrow e(\nabla y) \quad \text { weakly in } L^{2}\left(\Omega, \mathbb{R}_{\mathrm{sym}}^{d \times d}\right), \\
& \liminf _{k \rightarrow \infty} \mathcal{H}^{d-1}\left(J_{y_{k}}\right) \geqq \mathcal{H}^{d-1}\left(J_{y}\right) .
\end{aligned}
$$

The lower semicontinuity result for the jump set can be generalized considering one-dimensional slices. Define $\theta_{\sigma}:[0, \infty) \rightarrow[0,1]$ by $\theta_{\sigma}(t)=\min \left\{\frac{t}{\sigma}, 1\right\}$ for $\sigma>0$ and additionally $\theta_{0} \equiv 1$. Let

$$
\hat{\mu}_{y}^{\sigma, \xi}(B):=\int_{\Pi^{\xi}} \int_{B^{\xi, s} \cap J_{\hat{y} \xi, s}} \theta_{\sigma}\left(\left|\left[\hat{y}^{\xi, s}\right](t)\right|\right) \mathrm{d} \mathcal{H}^{0}(t) \mathrm{d} \mathcal{H}^{d-1}(s)
$$

for all Borel sets $B \subset \Omega$.

Lemma 3.3. Let $\left(y_{k}\right)_{k}$ be a sequence in $\operatorname{SSBD}^{2}(\Omega)$ converging to a function $y \in$ $\operatorname{GSBD}^{2}(\Omega)$ in the sense of (3.4). Then

$$
\hat{\mu}_{y}^{\sigma, \xi}(U) \leqq \liminf _{k \rightarrow \infty} \hat{\mu}_{y_{k}}^{\sigma, \xi}(U)
$$

for all $\sigma \geqq 0$, every $\xi \in S^{d-1}$ and for all open sets $U \subset \Omega$.

Proof. As $y_{k} \rightarrow y$ in the sense of (3.4), we may assume that $\left(\hat{y}_{k}\right)^{\xi, s} \rightarrow \hat{y}^{\xi, s}$ in $G S B V\left(U^{\xi, s}\right)$ for $\mathcal{H}^{d-1}$-almost every $s \in U^{\xi}:=\left\{s \in \Pi^{\xi}: U^{\xi, s} \neq \emptyset\right\}$. This is one of the essential steps in the proof of Theorem 3.2 (cf. [18, Theorem 11.3] or [4, Theorem 1.1] for an elaborated proof in the $S B D$-setting). The desired claim now follows from the corresponding lower semicontinuity result for $G S B V$ functions (see for example [3, Theorem 4.36]) and Fatou's lemma.

We briefly note that using the area formula (see for example [3, Theorem 2.71])) and fine properties of $G S B D$ functions (see [18]), $\hat{\mu}_{y}^{\sigma, \xi}(B)$ can be written equivalently as

$$
\hat{\mu}_{y}^{\sigma, \xi}(B)=\int_{J_{y}^{\xi} \cap B} \theta_{\sigma}(|[y] \cdot \xi|)\left|\nu_{y} \cdot \xi\right| \mathrm{d} \mathcal{H}^{d-1}
$$

for all $\sigma \geqq 0$, for all $\xi \in S^{d-1}$ and all Borel sets $B \subset \Omega$ (see also [18, Remark 9.3]). Finally, we recall a density result in GSBD (see [34]). 
Theorem 3.4. Let $y \in G S B D^{2}(\Omega) \cap L^{2}(\Omega)$. Then there exists a sequence $y_{k} \in$ $S B V^{2}(\Omega)$ such that each $J_{y_{k}}$ is contained in the union of a finite number of closed connected pieces of $C^{1}$-hypersurfaces, each $y_{k}$ belongs to $W^{1, \infty}\left(\Omega \backslash \overline{J_{y_{k}}}, \mathbb{R}^{d}\right)$ and the following properties hold:

$$
\begin{aligned}
& \text { (i) }\left\|y_{k}-y\right\|_{L^{2}(\Omega)} \rightarrow 0 ; \\
& \text { (ii) }\left\|e\left(\nabla y_{k}\right)-e(\nabla y)\right\|_{L^{2}(\Omega)} \rightarrow 0 ; \\
& \text { (iii) } \mathcal{H}^{d-1}\left(J_{y_{k}} \triangle J_{y}\right) \rightarrow 0 .
\end{aligned}
$$

\subsection{Caccioppoli Partitions}

Let $\Omega \subset \mathbb{R}^{d}$ open and $E \subset \Omega$ measurable. For $t \in[0,1]$ we define the points of density $t$ by

$$
E^{t}=\left\{x \in \mathbb{R}^{d}: \lim _{\varrho \downarrow 0} \frac{\left|E \cap B_{\varrho}(x)\right|}{\left|B_{\varrho}(x)\right|}=t\right\}
$$

(see [3, Definition 3.60]). By $\partial^{*} E=\mathbb{R}^{d} \backslash\left(E^{0} \cup E^{1}\right)$ we denote the essential boundary of $E$ and $\mathcal{H}^{1}\left(\partial^{*} E \cap \Omega\right)$ denotes the perimeter of $E$ in $\Omega$ (cf. [3, (3.62)]).

We say a partition $\mathcal{P}=\left(P_{j}\right)_{j \in \mathbb{N}}$ of $\Omega$ is a Caccioppoli partition of $\Omega$ if $\sum_{j} \mathcal{H}^{1}\left(\partial^{*} P_{j}\right)<+\infty$. We say a partition is ordered if $\left|P_{i}\right| \geqq\left|P_{j}\right|$ for $i \leqq j$. In the whole paper we will always tacitly assume that partitions are ordered. Given a rectifiable set $S$ we say that a Caccioppoli partition is subordinated to $S$ if (up to an $\mathcal{H}^{d-1}$-negligible set) the essential boundary $\partial^{*} P_{j}$ of $P_{j}$ is contained in $S$ for every $j \in \mathbb{N}$.

The local structure of Caccioppoli partitions can be characterized as follows (see [3, Theorem 4.17]).

Theorem 3.5. Let $\left(P_{j}\right)_{j}$ be a Caccioppoli partition of $\Omega$. Then

$$
\bigcup_{j}\left(P_{j}\right)^{1} \cup \bigcup_{i \neq j} \partial^{*} P_{i} \cap \partial^{*} P_{j}
$$

contains $\mathcal{H}^{d-1}$-almost all of $\Omega$, where $\left(P_{j}\right)^{1}$ as defined in (3.7).

Essentially, the theorem states that $\mathcal{H}^{d-1}$-almost every point of $\Omega$ either belongs to exactly one element of the partition or to the intersection of exactly two sets $\partial^{*} P_{i}$, $\partial^{*} P_{j}$. We now state a compactness result for ordered Caccioppoli partitions (see [3, Theorem 4.19, Remark 4.20]).

Theorem 3.6. Let $\Omega \subset \mathbb{R}^{d}$ open, bounded with Lipschitz boundary. Let $\mathcal{P}_{i}=$ $\left(P_{j, i}\right)_{j}, i \in \mathbb{N}$, be a sequence of ordered Caccioppoli partitions of $\Omega$ fulfilling $\sup _{i} \sum_{j} \mathcal{H}^{d-1}\left(\partial^{*} P_{j, i}\right)<\infty$. Then there exists a Caccioppoli partition $\mathcal{P}=\left(P_{j}\right)_{j}$ and a not relabeled subsequence such that $\left|P_{j, i} \triangle P_{j}\right| \rightarrow 0$ for all $j \in \mathbb{N}$ as $i \rightarrow \infty$.

We will also use the fact that $\left|P_{j, i} \Delta P_{j}\right| \rightarrow 0$ for all $j \in \mathbb{N}$ is equivalent to $\sum_{j}\left|P_{j, i} \triangle P_{j}\right| \rightarrow 0$. Caccioppoli partitions are naturally associated to piecewise constant functions. We say $y: \Omega \rightarrow \mathbb{R}^{m}$ is piecewiese constant in $\Omega$ if there exists a Caccioppoli partition $\left(P_{j}\right)_{j}$ of $\Omega$ and a sequence $\left(t_{j}\right)_{j} \subset \mathbb{R}^{m}$ such that $y=\sum_{j} t_{j} \chi_{P_{j}}$. We close this section with a compactness result for piecewise constant functions (see [3, Theorem 4.25]). 
Theorem 3.7. Let $\Omega \subset \mathbb{R}^{d}$ open, bounded with Lipschitz boundary. Let $\left(y_{i}\right)_{i} \subset$ $\operatorname{SBV}\left(\Omega, \mathbb{R}^{m}\right)$ be a sequence of piecewise constant functions such that $\sup _{i}\left(\left\|y_{i}\right\|_{\infty}+\right.$ $\left.\mathcal{H}^{d-1}\left(J_{y_{i}}\right)\right)<\infty$. Then there exists a not relabeled subsequence converging in measure to a piecewise constant function $y$.

\subsection{Rigidity Estimates}

In this section we first recall a geometric rigidity result obtained in the framework of nonlinear elasticity and a piecewise rigidity estimate for brittle materials for the sake of completeness. Afterwards we introduce a quantitative result in $S B D$ adapted for Griffith energies of the form (2.3) which will be the starting point for our analysis.

We begin with the quantitative geometric rigidity result by FRIESECKE ET AL. [32] which generalizes the classical Liouville theorem.

Theorem 3.8. Let $\Omega \subset \mathbb{R}^{d}$ a (connected) Lipschitz domain and $1<p<\infty$. Then there exists a constant $C=C(\Omega, p)$ such that for any $y \in W^{1, p}\left(\Omega, \mathbb{R}^{d}\right)$ there is a rotation $R \in S O(d)$ such that

$$
\|\nabla y-R\|_{L^{p}(\Omega)} \leq C\|\operatorname{dist}(\nabla y, S O(d))\|_{L^{p}(\Omega)} .
$$

In the theory of fracture mechanics the problem is more involved as global rigidity can fail if the crack disconnects the body. CHAmbolle, Giacomini AND PonsigLione [15] have proved the following qualitative result for brittle materials which do not store elastic energy (that is $\nabla y \in S O(d)$ almost everywhere in $\Omega$ ):

Theorem 3.9. Let $y \in S B V(\Omega)$ such that $\mathcal{H}^{d-1}\left(J_{y}\right)<+\infty$ and $\nabla y \in S O(d)$ almost everywhere in $\Omega$. Then $y$ is a collection of an at most countable family of rigid deformations, that is, there exists a Caccioppoli partition $\mathcal{P}=\left(P_{j}\right)_{j}$ subordinated to $J_{y}$ such that

$$
y(x)=\sum_{j}\left(R_{j} x+b_{j}\right) \chi_{P_{j}}(x),
$$

where $R_{j} \in S O(d)$ and $b_{j} \in \mathbb{R}^{d}$.

Loosely speaking, the result states that the only way that rigidity may fail is that the body is divided into at most countably many parts each of which subject to a different rigid motion. We briefly note that there is an analogous result in the geometrically linear setting (see [15, Theorem A.1]). A function $u \in S B D(\Omega)$ with $\mathcal{H}^{d-1}\left(J_{u}\right)<+\infty$ and $e(\nabla u)=0$ almost everywhere in $\Omega$ has the form $u(x)=\sum_{j}\left(A_{j} x+b_{j}\right) \chi_{P_{j}}(x)$ for $A_{j} \in \mathbb{R}_{\text {skew }}^{d \times d}$ and $b_{j} \in \mathbb{R}^{d}$.

We now introduce a quantitative $S B D$-rigidity result which may be seen as a suitable combination of the above estimates and is tailor-made for general Griffith functionals of the form (2.3) where both energy forms are coexistent (see [28, Theorem 2.1, Remark 2.2]). Let $\Omega_{\rho}=\{x \in \Omega$ : $\operatorname{dist}(x, \partial \Omega)>\rho\}$ for $\rho>0$. Recall (2.1), (2.3) and introduce an auxiliary energy functional by

$$
E_{\varepsilon}^{\rho}(y, U)=\frac{1}{\varepsilon} \int_{U} W(\nabla y(x)) \mathrm{d} x+\int_{J_{y} \cap U} f_{\varepsilon}^{\rho}(|[y](x)|) \mathrm{d} \mathcal{H}^{1}(x)
$$


for $\rho>0, \varepsilon>0$ and $U \subset \Omega$, where $f_{\varepsilon}^{\rho}(t):=\min \left\{\frac{t}{\sqrt{\varepsilon} \rho}, 1\right\}$. Recall the definition $e(G)=\frac{G+G^{T}}{2}$ for all $G \in \mathbb{R}^{2 \times 2}$.

Theorem 3.10. Let $\Omega \subset \mathbb{R}^{2}$ open, bounded with Lipschitz boundary. Let $M>0$ and $0<\eta, \rho<1$. Then there are a universal constant $c>0$, constants $\bar{C}=$ $\bar{C}(\Omega, M, \eta)>0, \hat{C}=\hat{C}(\Omega, M, \eta, \rho)>0$, and $\varepsilon_{0}=\varepsilon_{0}(M, \eta, \rho)>0$ such that the following holds for all $0<\varepsilon \leqq \varepsilon_{0}$ : for each $y \in S B V_{M}(\Omega)$ with $\mathcal{H}^{1}\left(J_{y}\right) \leqq M$ and $\int_{\Omega} \operatorname{dist}^{2}(\nabla y, S O(2)) \leqq M \varepsilon$ there is an open set $\Omega_{y} \subset \Omega$ and a modification $\hat{y} \in S B V_{c M}(\Omega)$ satisfying

(i) $\|\hat{y}-y\|_{L^{2}\left(\Omega_{y}\right)}^{2}+\|\nabla \hat{y}-\nabla y\|_{L^{2}\left(\Omega_{y}\right)}^{2} \leqq \bar{C} \varepsilon \rho, \quad\left|\Omega \backslash \Omega_{y}\right| \leqq \bar{C} \rho$, and

(ii) $E_{\varepsilon}^{\rho}\left(\hat{y}, \Omega_{\rho}\right) \leqq E_{\varepsilon}(y)+\bar{C} \rho$

with the following properties: We find a Caccioppoli partition $\mathcal{P}=\left(P_{j}\right)_{j}$ of $\Omega_{\rho}$ with $\sum_{j} \mathcal{H}^{1}\left(\partial^{*} P_{j} \cap \Omega_{\rho}\right) \leqq \bar{C}$ and for each $P_{j}$ a corresponding rigid motion $R_{j} \cdot+b_{j}, R_{j} \in S O(2)$ and $b_{j} \in \mathbb{R}^{2}$, such that the function $u: \Omega \rightarrow \mathbb{R}^{2}$ defined by

$$
u(x):= \begin{cases}\hat{y}(x)-\left(R_{j} x+b_{j}\right) & \text { for } x \in P_{j} \\ 0 & \text { for } x \in \Omega \backslash \Omega_{\rho}\end{cases}
$$

satisfies the estimates

$$
\begin{array}{cc}
\text { (i) } \mathcal{H}^{1}\left(J_{u}\right) \leqq \bar{C}, & \text { (ii) }\|u\|_{L^{2}\left(\Omega_{\rho}\right)}^{2} \leqq \hat{C} \varepsilon \\
\text { (iii) } \sum_{j}\left\|e\left(R_{j}^{T} \nabla u\right)\right\|_{L^{2}\left(P_{j}\right)}^{2} \leqq \hat{C} \varepsilon, & \text { (iv) }\|\nabla u\|_{L^{2}\left(\Omega_{\rho}\right)}^{2} \leqq \hat{C} \varepsilon^{1-\eta}
\end{array}
$$

We remark that we get a sufficiently strong bound only for the symmetric part of the gradient (see (3.9)(iii)) which is not surprising due to the fact that there is no direct analogue of Korn's inequality in $S B V$. However, there is at least a weaker bound on the full absolutely continuous part of the gradient $\nabla u$ (see (3.9)(iv)) which will essentially be needed to estimate the elastic part of the energy in the passage to the linearized theory (see (4.9) and (4.10) below). In particular, it will allow us to obtain (2.6)(ii) after modification of $u$ on a set of small measure.

Furthermore, let us briefly note that the uniform bound on the gradient (see (2.1)) in the setting of the nonlinear model is only needed for the application of the rigidity estimate. The condition essentially ensures that the elastic energy cannot concentrate on scales being much smaller than $\varepsilon$. In particular, this is a natural assumption in the investigation of discrete systems, where $\varepsilon$ may be interpreted as the typical interatomic distance.

Remark 3.11. (i) Estimate (3.9)(ii) can be refined. Indeed, we obtain

$$
\begin{aligned}
& \text { (i) } \frac{1}{\varepsilon} \int_{\Omega_{\rho}} W(\nabla \hat{y}(x)) \mathrm{d} x \leqq \frac{1}{\varepsilon} \int_{\Omega} W(\nabla y(x)) \mathrm{d} x+\bar{C} \rho \text {, and } \\
& \text { (ii) } \sum_{j} \frac{1}{2} \mathcal{H}^{1}\left(\partial^{*} P_{j} \cap \Omega_{\rho}\right)+\int_{J_{\hat{y}} \backslash \bigcup_{j} \partial^{*} P_{j}} f_{\varepsilon}^{\rho}(|[\hat{y}]|) \mathrm{d} \mathcal{H}^{1} \leqq \mathcal{H}^{1}\left(J_{y}\right)+\bar{C} \rho .
\end{aligned}
$$


We remark that it is indispensable to allow for a small modification of the deformation in Theorem 3.10 in order to guarantee the sharp energy estimate (3.11)(ii).

(ii) To derive (3.10)(iii) one essentially shows

$$
\|\nabla u\|_{L^{4}\left(\Omega_{\rho}\right)}^{4}=\sum_{j}\left\|\nabla \hat{y}-R_{j}\right\|_{L^{4}\left(P_{j}\right)}^{4} \leqq \hat{C} \varepsilon .
$$

The claim then follows from (2.2), (3.11)(i), $\|\operatorname{dist}(\nabla y, S O(2))\|_{L^{2}(\Omega)}^{2} \leqq M \varepsilon$ and the linearization formula (see $[32,(3.20)]$ )

$$
\left|e\left(R^{T} G-\mathbf{I d}\right)\right|=\operatorname{dist}(G, S O(2))+O\left(|G-R|^{2}\right)
$$

for $G \in \mathbb{R}^{2 \times 2}$ and $R \in S O(2)$, where Id denotes the identity matrix.

\section{Compactness of Rescaled Configurations}

This section is devoted to the proof of the main compactness result given in Theorem 2.2. Moreover, we also show that Theorem 2.2 provides an alternative proof of the piecewise rigidity result stated in Theorem 3.9.

\subsection{Preparations}

For the compactness theorem in GSBD (see Theorem 3.2) it is necessary that the integral for some integrand $\psi$ with $\lim _{t \rightarrow \infty} \psi(t)=\infty$ is uniformly bounded. We first give a simple criterion for the existence of such a function which is, loosely speaking, based on the condition that the functions coincide in a certain sense on the bulk part of the domain.

Lemma 4.1. For every increasing sequence $\left(b_{i}\right)_{i} \subset(0, \infty)$ with $b_{i} \rightarrow \infty$ there is an increasing concave function $\psi:[0, \infty) \rightarrow[0, \infty)$ with $\lim _{t \rightarrow \infty} \psi(t)=\infty$ and $\psi\left(b_{i}\right) \leqq 2^{i}$ for all $i \in \mathbb{N}$.

Proof. Let $f:[0, \infty) \rightarrow[0, \infty)$ be the function with $f(0)=0, f\left(b_{i}\right)=2^{i}$ which is affine on each segment $\left[b_{i}, b_{i+1}\right]$. Clearly, $f$ is increasing and satisfies $f(t) \rightarrow \infty$ for $t \rightarrow \infty$, but is possibly not concave. We now construct $\psi$ and first let $\psi=f$ on $\left[0, b_{1}\right]$. Assume $\psi$ has been defined on $\left[0, b_{i}\right]$ and that $\psi$ is increasing, concave, satisfies $\psi \leqq f$ and $\psi\left(b_{i}\right)=f\left(b_{i}\right)=2^{i}$. If $f^{\prime}\left(b_{i}-\right) \geqq f^{\prime}\left(b_{i}+\right)$, we set $\psi=f$ on $\left[b_{i}, b_{i+1}\right]$. Here, $f^{\prime}(t \pm)$ denote the one-sided limits of the derivative at point $t$. This implies that $\psi$ is concave on $\left[0, b_{i+1}\right]$ since $\psi^{\prime}\left(b_{i}-\right) \geqq f^{\prime}\left(b_{i}-\right)$.

Otherwise, we let $\psi(t)=f\left(b_{i}\right)+f^{\prime}\left(b_{i}-\right)\left(t-b_{i}\right)$ for $t \in\left[b_{i}, \bar{t}\right]$, where $\bar{t}$ is the smallest value larger than $b_{i}$ such that $f(\bar{t})=f\left(b_{i}\right)+f^{\prime}\left(b_{i}-\right)\left(\bar{t}-b_{i}\right)$. If $\bar{t}$ does not exist, we are done. If $\bar{t}$ exists, we assume $\bar{t} \in\left(b_{j-1}, b_{j}\right]$ and define $\psi=f$ on $\left[\bar{t}, b_{j}\right]$. Note that if $\bar{t} \in\left(b_{j-1}, b_{j}\right)$, we have $\psi^{\prime}(\bar{t}-) \geqq \psi^{\prime}(\bar{t}+)$ since $f$ is affine on $\left[b_{j-1}, b_{j}\right]$ and $\psi<f$ on $\left(b_{i}, \bar{t}\right)$. Thus, $\psi$ is concave on $\left[0, b_{j}\right]$.

Repeating the construction we end up with an increasing concave function $\psi$ with $\psi \leqq f$ and $\psi(t) \rightarrow \infty$ for $t \rightarrow \infty$. 
Lemma 4.2. Let $\Omega \subset \mathbb{R}^{2}$ and let $\left(y^{l}\right)_{l} \subset L^{1}(\Omega)$ be a sequence satisfying $\left|\Omega \backslash \bigcup_{n \in \mathbb{N}} \bigcap_{l \geqq n}\left\{\left|y^{n}-y^{l}\right| \leqq 1\right\}\right|=0$. Then there is a not relabeled subsequence such that

$$
\int_{\Omega} \psi\left(\left|y^{l}\right|\right) \leqq C
$$

for a constant $C>0$ independent ofl, where $\psi$ is an increasing continuous function with $\lim _{t \rightarrow \infty} \psi(t)=\infty$.

Proof. Define $C_{l}:=\max _{1 \leqq i \leqq l}\left\|y^{i}\right\|_{L^{1}(\Omega)}$ for all $l \in \mathbb{N}$. Let $A_{n}=\bigcap_{l \geqq n}\left\{\mid y^{n}-\right.$ $\left.y^{l} \mid \leqq 1\right\}$ and set $B_{1}=A_{1}$ as well as $B_{n}=A_{n} \backslash \bigcup_{m=1}^{n-1} B_{m}$ for all $n \in \mathbb{N}$. The sets $\left(B_{n}\right)_{n}$ are pairwise disjoint with $\sum_{n}\left|B_{n}\right|=|\Omega|$. We choose $0=n_{1}<n_{2}<\ldots$ such that $\sum_{1 \leqq n \leqq n_{i}} \frac{\left|B_{n}\right|}{|\Omega|} \geqq 1-4^{-i}$. We let $B^{i}=\bigcup_{n=n_{i}+1}^{n_{i+1}} B_{n}$ and observe $\left|B^{i}\right| \leqq$ $4^{-i}|\Omega|$.

We pass to the subsequence $\left(n_{i}\right)_{i} \subset \mathbb{N}$ and choose $E^{i} \supset B^{i}$ such that $\left|E^{i}\right|=$ $4^{-i}|\Omega|$. Let $b_{i}=\frac{C_{n_{i+1}}}{\left|E^{i}\right|}+2=4^{i} \frac{C_{n_{i+1}}}{|\Omega|}+2$ for $i \in \mathbb{N}$ and note that $\left(b_{i}\right)_{i}$ is increasing with $b_{i} \rightarrow \infty$. By Lemma 4.1 we get an increasing concave function $\psi:[0, \infty) \rightarrow[0, \infty)$ with $\lim _{t \rightarrow \infty} \psi(t)=\infty$ and $\psi\left(b_{i}\right) \leqq 2^{i}$ for all $i \in \mathbb{N}$. Clearly, $\psi$ is also continuous.

For $\hat{B}^{i}:=\Omega \backslash \bigcup_{n=1}^{n_{i}} B_{n}$ we have $\left|\hat{B}^{i}\right| \leqq 4^{-i}|\Omega|$ and choose $\hat{E}^{i} \supset \hat{B}^{i}$ with $\left|\hat{E}^{i}\right|=4^{-i}|\Omega|$. We then obtain $\frac{C_{n_{i}}}{\left|\hat{E}^{i}\right|}=4^{i} \frac{C_{n_{i}}}{|\Omega|} \leqq b_{i}$. Now let $l=n_{i}$. Using Jensen's inequality, the definition of the sets $B^{i},\left\|y^{l}\right\|_{L^{1}(\Omega)} \leqq C_{l}$ and the monotonicity of $\psi$ we compute

$$
\begin{aligned}
\int_{\Omega} \psi\left(\left|y^{l}\right|\right) & =\sum_{1 \leqq j \leqq i-1} \int_{B^{j}} \psi\left(\left|y^{l}\right|\right)+\int_{\hat{B}^{i}} \psi\left(\left|y^{l}\right|\right) \\
& \leqq \sum_{1 \leqq j \leqq i-1} \int_{B^{j}} \psi\left(\left|y^{n_{j+1}}\right|+2\right)+\int_{\hat{B}^{i}} \psi\left(\left|y^{l}\right|\right) \\
& \leqq \sum_{1 \leqq j \leqq i-1}\left|E^{j}\right| \psi\left(f_{E^{j}}\left|y^{n_{j+1}}\right|+2\right)+\left|\hat{E}^{i}\right| \psi\left(f_{\hat{E}^{i}}\left|y^{l}\right|\right) \\
& \leqq \sum_{1 \leqq j \leqq i-1} 4^{-j}|\Omega| 2^{j}+4^{-i}|\Omega| 2^{i} \leqq|\Omega| \sum_{j \in \mathbb{N}} 2^{-j} .
\end{aligned}
$$

As the estimate is independent of $l \in\left(n_{i}\right)_{i}$, this yields $\int_{\Omega} \psi\left(\left|y^{l}\right|\right) \leqq C$ uniformly in $l$, as desired.

\subsection{Proof of Theorem 2.2}

Now we are in a position to give the proof of the main compactness result. In the first part we show that (2.6)-(2.8) hold.

Proof of Theorem 2.2, part 1. Let $\left(\varepsilon_{k}\right)_{k}$ be a sequence with $\varepsilon_{k} \rightarrow 0$. Let $y_{k} \in$ $S B V_{M}(\Omega)$ with $E_{\varepsilon_{k}}\left(y_{k}\right) \leqq C$ be given. Possibly passing to a larger $M$, we get $\left\|\operatorname{dist}\left(\nabla y_{k}, S O(2)\right)\right\|_{L^{2}(\Omega)}^{2} \leqq M \varepsilon_{k}$ by (2.2) and $\mathcal{H}^{1}\left(J_{y_{k}}\right) \leqq M$ for all $k \in \mathbb{N}$. In the following generic constants only depending on $\Omega$ and $M$ will be denoted by $C$. 
Step I: Choose $\rho_{0}>0$ small and let $\rho_{l}=2^{-3 l} \rho_{0}$ for all $l \in \mathbb{N}$. We apply Theorem 3.10 for $\rho=\rho_{l}$ and $\eta=\frac{1}{5}$ (the choice of $\eta$ is related to the exponent $-\frac{1}{8}$ in (2.6)(ii)). Denote by $c, \bar{C}=\bar{C}(\Omega, M, \eta), \hat{C}_{l}=\hat{C}_{l}\left(\Omega, M, \eta, \rho_{l}\right)$ the constants in Theorem 3.10. For each $l \in \mathbb{N}$ there exists $\kappa_{l}=\kappa_{l}\left(M, \eta, \rho_{l}\right)$ such that for $k \geqq \kappa_{l}$ we find modifications $y_{k}^{l} \in S B V_{c M}\left(\Omega, \mathbb{R}^{2}\right)$ with $E_{\varepsilon_{k}}^{\rho_{l}}\left(y_{k}^{l}, \Omega_{\rho_{l}}\right) \leqq E_{\varepsilon_{k}}\left(y_{k}\right)+\bar{C} \rho_{l}$ and

$$
\left\|y_{k}^{l}-y_{k}\right\|_{L^{2}\left(\Omega_{k}^{l}\right)}^{2}+\left\|\nabla y_{k}^{l}-\nabla y_{k}\right\|_{L^{2}\left(\Omega_{k}^{l}\right)}^{2} \leqq \bar{C} \varepsilon_{k} \rho_{l}
$$

where $\Omega_{k}^{l}:=\Omega_{y_{k}^{l}}$ with $\left|\Omega \backslash \Omega_{k}^{l}\right| \leqq \bar{C} \rho_{l}$. We further get Caccioppoli partitions $\left(P_{j}^{k, l}\right)_{j}$ of $\Omega_{\rho_{l}}$ with $\sum_{j} \mathcal{H}^{1}\left(\partial^{*} P_{j}^{k, l} \cap \Omega_{\rho_{l}}\right) \leqq \bar{C}$ and corresponding piecewise rigid motions $T_{k}^{l}:=\sum_{j}\left(R_{j}^{k, l} \cdot+b_{j}^{k, l}\right) \chi_{P_{j}^{k, l}}+\mathbf{i d} \chi_{\Omega \backslash \Omega_{\rho_{l}}}$ such that the functions $v_{k}^{l}: \Omega \rightarrow \mathbb{R}^{2}$ defined by

$$
v_{k}^{l}(x)= \begin{cases}\frac{1}{\sqrt{\varepsilon_{k}}}\left(R_{j}^{k, l}\right)^{T}\left(y_{k}^{l}(x)-\left(R_{j}^{k, l} x+b_{j}^{k, l}\right)\right) & \text { for } x \in P_{j}^{k, l}, j \in \mathbb{N}, \\ 0 & \text { else }\end{cases}
$$

satisfy by (3.10)

$$
\mathcal{H}^{1}\left(J_{v_{k}^{l}}\right) \leqq \bar{C}, \quad\left\|v_{k}^{l}\right\|_{L^{2}(\Omega)}+\left\|e\left(\nabla v_{k}^{l}\right)\right\|_{L^{2}(\Omega)} \leqq \hat{C}_{l}, \quad\left\|\nabla v_{k}^{l}\right\|_{L^{2}(\Omega)}^{2} \leqq \hat{C}_{l} \varepsilon_{k}^{-1 / 5} .
$$

We recall $\left\|y_{k}^{l}\right\|_{\infty} \leqq c M$ for all $k \geqq \kappa_{l}$. Thus, possibly passing to other (not relabeled) constants $b_{j}^{k, l}$ in (4.3), we can assume that $\left|b_{j}^{k, l}\right| \leqq C M$ for $C=C(\Omega, c)$ and that (4.4) still holds. Each partition may be extended to $\Omega$ by adding the element $\Omega \backslash \Omega_{\rho_{l}}$. As for $\rho_{0}$ small enough we get $\mathcal{H}^{1}\left(\partial \Omega_{\rho_{l}}\right) \leqq C \mathcal{H}^{1}(\partial \Omega)$ (see [24, Theorem 4.1]) for all $l \in \mathbb{N}$, there is $C=C(\bar{C}, \Omega)$ such that

$$
\sum_{j} \mathcal{H}^{1}\left(\partial^{*} P_{j}^{k, l}\right) \leqq C
$$

Step II: Using a diagonal argument we get a (not relabeled) subsequence of $\left(\varepsilon_{k}\right)_{k}$ such that by Theorem 3.2 for every $l \in \mathbb{N}$ we find $v^{l} \in G S B D^{2}(\Omega)$ with $v_{k}^{l} \rightarrow v^{l}$ almost every where in $\Omega$ and $e\left(\nabla v_{k}^{l}\right) \rightarrow e\left(\nabla v^{l}\right)$ weakly in $L^{2}\left(\Omega, \mathbb{R}_{\mathrm{sym}}^{2 \times 2}\right)$

for $k \rightarrow \infty$. By Theorem 3.6, Theorem 3.7, (4.5) and the fact that $\left|b_{j}^{k, l}\right| \leqq C M$ we obtain an (ordered) partition $\left(P_{j}^{l}\right)_{j}$ of $\Omega$ with $\sum_{j} \mathcal{H}^{1}\left(\partial^{*} P_{j}^{l}\right) \leqq C$ and a piecewise rigid motion $T^{l}:=\sum_{j}\left(R_{j}^{l} \cdot+b_{j}^{l}\right) \chi_{P_{j}^{l}}$ such that for all $l \in \mathbb{N}$ we get (again up to a subsequence) $\left|P_{j}^{k, l} \triangle P_{j}^{l}\right| \rightarrow 0, R_{j}^{k, l} \rightarrow R_{j}^{l}$, and $b_{j}^{k, l} \rightarrow b_{j}^{l}$ for all $j \in \mathbb{N}$ as $k \rightarrow \infty$. This also implies

$$
\sum_{j}\left|P_{j}^{k, l} \triangle P_{j}^{l}\right|+\left\|T_{k}^{l}-T^{l}\right\|_{L^{2}(\Omega)}+\left\|\nabla T_{k}^{l}-\nabla T^{l}\right\|_{L^{2}(\Omega)} \rightarrow 0
$$


for $k \rightarrow \infty$. We now show that

$$
\left\|v^{l}\right\|_{L^{1}(\Omega)} \leqq C\left\|v^{l}\right\|_{L^{2}(\Omega)} \leqq C \hat{C}_{l}, \quad \mathcal{H}^{1}\left(J_{v^{l}}\right) \leqq \bar{C}, \quad\left\|e\left(\nabla v^{l}\right)\right\|_{L^{2}(\Omega)}^{2} \leqq C .
$$

The first two claims follow directly from (4.4) and (3.4). To see the third estimate, we let $\phi_{k}^{l}(x):=\chi_{\left[0, \varepsilon_{k}^{-1 / 8}\right]}\left(\left|\nabla v_{k}^{l}(x)\right|\right)($ cf. (2.6)(ii)). Moreover, we obtain by an elementary computation ( $\mathrm{cf} .(3.12)) \operatorname{dist}^{2}(G, S O(2))=\left|e\left(R^{T} G-\mathbf{I d}\right)\right|^{2}+\omega_{\text {dist }}\left(R^{T} G-\mathbf{I d}\right)$ for $G \in \mathbb{R}^{2 \times 2}, R \in S O(2)$ with $\sup \left\{|G|^{-3} \omega_{\text {dist }}(G):|G| \leqq 1\right\} \leqq C$. We compute by (2.2) and (4.3)

$$
\begin{aligned}
C & \geqq E_{\varepsilon_{k}}^{\rho_{l}}\left(y_{k}^{l}, \Omega_{\rho_{l}}\right) \geqq \frac{C}{\varepsilon_{k}} \int_{\Omega_{\rho_{l}}} \operatorname{dist}^{2}\left(\nabla y_{k}^{l}, S O(2)\right) \\
& \geqq \frac{C}{\varepsilon_{k}} \sum_{j} \int_{P_{j}^{k, l} \cap \Omega_{\rho_{l}}} \phi_{k}^{l}\left(\left|e\left(\left(R_{j}^{k, l}\right)^{T} \nabla y_{k}^{l}-\mathbf{I d}\right)\right|^{2}+\omega_{\operatorname{dist}}\left(\left(R_{j}^{k, l}\right)^{T} \nabla y_{k}^{l}-\mathbf{I d}\right)\right) \\
& =C \int_{\Omega} \phi_{k}^{l}\left(\left|e\left(\nabla v_{k}^{l}\right)\right|^{2}+\frac{1}{\varepsilon_{k}} \omega_{\operatorname{dist}}\left(\sqrt{\varepsilon_{k}} \nabla v_{k}^{l}\right)\right) .
\end{aligned}
$$

The second term of the integral can be estimated by

$$
\int_{\Omega} \phi_{k}^{l} \frac{1}{\varepsilon_{k}} \omega_{\text {dist }}\left(\sqrt{\varepsilon_{k}} \nabla v_{k}^{l}\right)=\int_{\Omega} \phi_{k}^{l} \sqrt{\varepsilon_{k}}\left|\nabla v_{k}^{l}\right|^{3} \frac{\omega_{\text {dist }}\left(\sqrt{\varepsilon_{k}} \nabla v_{k}^{l}\right)}{\left|\sqrt{\varepsilon_{k}} \nabla v_{k}^{l}\right|^{3}} \leqq C \varepsilon_{k}^{\frac{1}{8}} \rightarrow 0 .
$$

As $e\left(\nabla v_{k}^{l}\right) \rightarrow e\left(\nabla v^{l}\right)$ weakly in $L^{2}(\Omega)$ and $\phi_{k}^{l} \rightarrow 1$ boundedly in measure on $\Omega$ by (4.4), it follows $\phi_{k}^{l} e\left(\nabla v_{k}^{l}\right) \rightarrow e\left(\nabla v^{l}\right)$ weakly in $L^{2}(\Omega)$. By lower semicontinuity we obtain $\left\|e\left(\nabla v^{l}\right)\right\|_{L^{2}(\Omega)}^{2} \leqq C$ for a constant particularly independent of $\rho_{l}$ which concludes (4.8).

Step III: We now want to pass to the limit $l \rightarrow \infty$. Similarly as in the argumentation leading to (4.7), by the compactness result for piecewise constant functions (see Theorem 3.7) we find a partition $\left(P_{j}\right)_{j}$ of $\Omega$ and a piecewise rigid motion $T:=\sum_{j}\left(R_{j} \cdot+b_{j}\right) \chi_{P_{j}}$ such that for a suitable (not relabeled) subsequence

$$
\sum_{j}\left|P_{j}^{l} \triangle P_{j}\right|+\left\|T^{l}-T\right\|_{L^{2}(\Omega)}+\left\|\nabla T^{l}-\nabla T\right\|_{L^{2}(\Omega)} \rightarrow 0
$$

for $l \rightarrow \infty$. Recalling (4.7) and using a diagonal argument we can choose a (not relabeled) subsequence of $\left(\rho_{l}\right)_{l}$ and afterwards of $\left(\varepsilon_{k}\right)_{k}$ such that for all $l$ we have

$$
\sum_{j}\left|P_{j}^{l} \triangle P_{j}\right| \leqq 2^{-l}, \quad \sum_{j}\left|P_{j}^{k, l} \triangle P_{j}^{l}\right| \leqq 2^{-l} \text { for all } k \geqq l .
$$

We see that the compactness result in GSBD cannot be applied directly on the sequence $\left(v^{l}\right)_{l}$ as the $L^{2}$ bound in (4.8) depends on $\rho_{l}$. We now show that by choosing the rigid motions on the elements of the partitions appropriately (see (4.3)) we can construct the sequence $\left(v^{l}\right)_{l}$ such that we obtain

$$
\left|\Omega \backslash \bigcup_{n \in \mathbb{N}} \bigcap_{m \geqq n}\left\{\left|v^{n}-v^{m}\right| \leqq 1\right\}\right|=0,
$$


and thus Lemma 4.2 is applicable.

Step IV: We fix $k \in \mathbb{N}$ and describe an iterative procedure to redefine $R_{j}^{k, l}, b_{j}^{k, l}$ for all $l$ with $k \geqq \kappa_{l}$ and $j \in \mathbb{N}$. Let $\tilde{v}_{k}^{1}=v_{k}^{1}$ as defined in (4.3) and assume $\tilde{v}_{k}^{l}$ with corresponding $\tilde{R}_{j}^{k, l}, \tilde{b}_{j}^{k, l}$ have been chosen (which possibly differ from $R_{j}^{k, l}, b_{j}^{k, l}$ ) such that (4.4) still holds possibly passing to a larger constant $\tilde{C}_{l}=\tilde{C}_{l}(\Omega, M, \eta, l)$. Let $I_{1}^{k, l}=\left\{j:\left|P_{j}^{k, l+1} \cap P_{j}^{k, l}\right| \geqq 4 \bar{C} \rho_{l}\right\}$ and $I_{2}^{k, l}=\mathbb{N} \backslash I_{1}^{k, l}$. Define

$$
\begin{aligned}
& \tilde{R}_{j}^{k, l+1}=\tilde{R}_{j}^{k, l}, \quad \tilde{b}_{j}^{k, l+1}=\tilde{b}_{j}^{k, l} \quad \text { for } j \in I_{1}^{k, l}, \\
& \tilde{R}_{j}^{k, l+1}=R_{j}^{k, l+1}, \quad \tilde{b}_{j}^{k, l+1}=b_{j}^{k, l+1} \quad \text { for } j \in I_{2}^{k, l} .
\end{aligned}
$$

Consider $j \in I_{1}^{k, l}$ and define $R_{j}^{\prime}=R_{j}^{k, l+1}-\tilde{R}_{j}^{k, l}, b_{j}^{\prime}=b_{j}^{k, l+1}-\tilde{b}_{j}^{k, l}, P_{j}^{\prime}=$ $P_{j}^{k, l+1} \cap P_{j}^{k, l} \cap \Omega_{k}^{l} \cap \Omega_{k}^{l+1}$ for shorthand. By the triangle inequality, (4.2) and (4.3) we get

$$
\begin{aligned}
\left\|R_{j}^{\prime} \cdot+b_{j}^{\prime}\right\|_{L^{2}\left(P_{j}^{\prime}\right)} & \leqq \sqrt{\varepsilon_{k}}\left(\left\|\tilde{v}_{k}^{l}\right\|_{L^{2}(\Omega)}+\left\|v_{k}^{l+1}\right\|_{L^{2}(\Omega)}\right)+\left\|y_{k}^{l}-y_{k}^{l+1}\right\|_{L^{2}\left(\Omega_{k}^{l} \cap \Omega_{k}^{l+1}\right)} \\
& \leqq \sqrt{\varepsilon_{k}}\left(\tilde{C}_{l}+\hat{C}_{l+1}+C \sqrt{\rho_{l}}+C \sqrt{\rho_{l+1}}\right) \leqq C_{l}^{\prime} \sqrt{\varepsilon_{k}}
\end{aligned}
$$

for a constant $C_{l}^{\prime}=C_{l}^{\prime}(\Omega, M, \eta, l)$, where in the penultimate step we used that (4.4) holds for $\tilde{v}_{k}^{l}$ and $v_{k}^{l+1}$. Herefrom we now derive $\left|R_{j}^{\prime}\right| \leqq C_{l}^{\prime} \sqrt{\varepsilon_{k}}$. Indeed, if $R_{j}^{\prime} \neq 0$, then $R_{j}^{\prime}$ is invertible and a short computation yields

$$
\frac{1}{\sqrt{2}}\left|R_{j}^{\prime}\right|\|\cdot-z\|_{L^{2}\left(P_{j}^{\prime} \backslash B_{\lambda}(z)\right)} \leqq\left\|R_{j}^{\prime} \cdot+b_{j}^{\prime}\right\|_{L^{2}\left(P_{j}^{\prime}\right)} \leqq C_{l}^{\prime} \sqrt{\varepsilon_{k}},
$$

where $z:=-\left(R_{j}^{\prime}\right)^{-1} b_{j}^{\prime}$ and $B_{\lambda}(z)$ denotes the ball with center $z$ and radius $\lambda=$ $\left(\pi^{-1} \bar{C} \rho_{l}\right)^{1 / 2}$. Then by definition of $I_{1}^{k, l}$ and $\left|\Omega \backslash \Omega_{k}^{l}\right| \leqq \bar{C} \rho_{l}$ we find $\left|P_{j}^{\prime} \backslash B_{\lambda}(z)\right| \geqq$ $\left|P_{j}^{k, l+1} \cap P_{j}^{k, l}\right|-\left|\Omega \backslash \Omega_{k}^{l}\right|-\left|\Omega \backslash \Omega_{k}^{l+1}\right|-\left|B_{\lambda}(z)\right| \geqq \bar{C} \rho_{l}$, which together with (4.16) implies the claim for $C_{l}^{\prime}$ sufficiently large.

Recalling (4.15) we then also find $\left|b_{j}^{\prime}\right| \leqq C_{l}^{\prime} \sqrt{\varepsilon_{k}}$ and summing over all components we derive

$$
\begin{aligned}
& \sum_{j \in I_{1}^{k, l}}\left(\left\|R_{j}^{\prime}\right\|_{L^{2}\left(P_{j}^{k, l+1}\right)}^{2}+\left\|R_{j}^{\prime}\right\|_{L^{4}\left(P_{j}^{k, l+1}\right)}^{4}+\left\|R_{j}^{\prime} \cdot+b_{j}^{\prime}\right\|_{L^{2}\left(P_{j}^{k, l+1}\right)}^{2}\right) \\
& \leqq \# I_{1}^{k, l} C_{l}^{\prime} \varepsilon_{k} \leqq \frac{|\Omega| C_{l}^{\prime}}{4 \bar{C} \rho_{l}} \varepsilon_{k},
\end{aligned}
$$

where in the last step we used the definition of $I_{1}^{k, l}$. Define $\tilde{v}_{k}^{l+1}$ as in (4.3) with $\tilde{R}_{j}^{k, l+1}, \tilde{b}_{j}^{k, l+1}$ instead of $R_{j}^{k, l+1}, b_{j}^{k, l+1}$. The previous estimate together with the fact that (4.4) holds for $v_{k}^{l+1}$ now shows (4.4) for $\tilde{v}_{k}^{l+1}$. Indeed, the estimates for $\left\|\tilde{v}_{k}^{l+1}\right\|_{L^{2}(\Omega)},\left\|\nabla \tilde{v}_{k}^{l+1}\right\|_{L^{2}(\Omega)}$ follow directly and for $\left\|e\left(\nabla \tilde{v}_{k}^{l+1}\right)\right\|_{L^{2}(\Omega)}$ we argue as in Remark 3.11(ii).

Note that as $\left(\hat{C}_{l}\right)_{l}$ also $\left(\tilde{C}_{l}\right)_{l}$ converges to infinity. For simplicity the modified functions and rigid motions will still be denoted by $v_{k}^{l}, R_{j}^{k, l}$ and $b_{j}^{k, l}$ in the following. 
By a diagonal argument we can choose a further (not relabeled) subsequence of $\left(\varepsilon_{k}\right)_{k}$ such that the modifications $v_{k}^{l}$ exist for all $l \in \mathbb{N}$ and $k \geqq l$.

Step $V$ : We define $A_{k, l}^{n}=\bigcap_{n \leqq m \leqq l}\left\{\left|v_{k}^{m}-v_{k}^{n}\right| \leqq \frac{1}{2}\right\}$ for all $n \in \mathbb{N}$ and $n \leqq l \leqq k$. If we show

$$
\left|\Omega \backslash A_{k, l}^{n}\right| \leqq C 2^{-n},
$$

then (4.13) follows. Indeed, for given $l \geqq n$ we can choose $K=K(l) \geqq l$ so large that $\left|\left\{\left|v_{K}^{m}-v^{m}\right|>\frac{1}{4}\right\}\right| \leqq 2^{-m}$ for all $n \leqq m \leqq l$ since $v_{k}^{m} \rightarrow v^{m}$ in measure for $k \rightarrow \infty$. This implies

$\left|\Omega \backslash \bigcap_{n \leqq m \leqq l}\left\{\left|v^{m}-v^{n}\right| \leqq 1\right\}\right| \leqq\left|\Omega \backslash A_{K, l}^{n}\right|+\sum_{n \leqq m \leqq l}\left|\left\{\left|v_{K}^{m}-v^{m}\right|>\frac{1}{4}\right\}\right| \leqq C 2^{-n}$.

Passing to the limit $l \rightarrow \infty$ we find $\left|\Omega \backslash \bigcap_{m \geqq n}\left\{\left|v^{m}-v^{n}\right| \leqq 1\right\}\right| \leqq C 2^{-n}$ and taking the union over all $n \in \mathbb{N}$ we derive (4.13).

To show (4.17) we proceed in two steps. Employing the redefinition of the piecewise rigid motions we first show that the set where $T_{k}^{m}, n \leqq m \leqq l$, differ is small. Afterwards, we use (4.2) to find that the set where $y_{k}^{m}, n \leqq m \leqq l$, differ is small. We define $B_{k, l}^{n}=\bigcap_{n \leqq m \leqq l}\left\{T_{k}^{m}=T_{k}^{n}\right\}$ for $k \geqq l \geqq n$ and prove that

$$
\left|\Omega \backslash B_{k, l}^{n}\right| \leqq C 2^{-n}
$$

for all $k \geqq l \geqq n$. To this end, consider $\left\{T_{k}^{m}=T_{k}^{m+1}\right\}$ for $n \leqq m \leqq l-1$ and first note that by (4.12) we have $\sum_{j}\left|P_{j}^{k, m+1} \triangle P_{j}^{k, m}\right| \leqq 3 \cdot 2^{-m}$. Define $J_{1} \subset \mathbb{N}$ such that $\left|P_{j}^{k, m+1}\right| \leqq 8 \bar{C} \rho_{m}$ for all $j \in J_{1}$ and let $J_{2} \subset \mathbb{N} \backslash J_{1}$ such that $\mid P_{j}^{k, m+1} \cap$ $P_{j}^{k, m}\left|>\frac{1}{2}\right| P_{j}^{k, m+1} \mid$ for all $j \in J_{2}$. Observe that $\left|P_{j}^{k, m+1}\right| \leqq 2\left|P_{j}^{k, m+1} \backslash P_{j}^{k, m}\right|$ for $j \in J_{3}:=\mathbb{N} \backslash\left(J_{1} \cup J_{2}\right)$. Using the isoperimetric inequality and the the fact that $\left(\rho_{m}\right)_{m} \subset\left(2^{-3 m} \rho_{0}\right)_{m}$ we find by (4.5)

$$
\sum_{j \in J_{1}}\left|P_{j}^{k, m+1}\right| \leqq\left(8 \bar{C} \rho_{m}\right)^{\frac{1}{2}} \sum_{j \in J_{1}}\left|P_{j}^{k, m+1}\right|^{\frac{1}{2}} \leqq C 2^{-m} \sum_{j} \mathcal{H}^{1}\left(\partial^{*} P_{j}^{k, m+1}\right) \leqq C 2^{-m}
$$

Due to the above construction of the rigid motions (see (4.14)) we obtain $\left\{T_{k}^{m}=\right.$ $\left.T_{k}^{m+1}\right\} \supset \bigcup_{j \in J_{2}}\left(P_{j}^{k, m+1} \cap P_{j}^{k, m}\right)$ and therefore

$$
\begin{aligned}
\left|\Omega \backslash\left\{T_{k}^{m}=T_{k}^{m+1}\right\}\right| & \leqq \sum_{j \in J_{2}}\left|P_{j}^{k, m+1} \backslash P_{j}^{k, m}\right|+\sum_{j \in J_{1} \cup J_{3}}\left|P_{j}^{k, m+1}\right| \\
& \leqq \sum_{j \in J_{2}}\left|P_{j}^{k, m+1} \backslash P_{j}^{k, m}\right|+\sum_{j \in J_{3}} 2\left|P_{j}^{k, m+1} \backslash P_{j}^{k, m}\right|+C 2^{-m} \\
& \leqq 2 \sum_{j}\left|P_{j}^{k, m+1} \triangle P_{j}^{k, m}\right|+C 2^{-m} \leqq C 2^{-m} .
\end{aligned}
$$

Summing over $n \leqq m \leqq l-1$ we establish (4.18). Now recalling (4.2), (4.18), $\left|\Omega \backslash \Omega_{k}^{l}\right| \leqq \bar{C} \rho_{l}$ and the fact that $\left(\rho_{l}\right)_{l} \subset\left(2^{-3 l} \rho_{0}\right)_{l}$ we find

$$
\left|\Omega \backslash A_{k, l}^{n}\right| \leqq\left|\Omega \backslash B_{k, l}^{n}\right|+\sum_{n \leqq m \leqq l-1}\left|\left\{\left|y_{k}^{m+1}-y_{k}^{m}\right|>2^{-m-1} \sqrt{\varepsilon_{k}}\right\}\right| \leqq C 2^{-n}
$$

for all $k \geqq l \geqq n$, as desired. 
Step VI: By (4.8) and (4.13) we can apply Lemma 4.2 on the sequence $\left(v^{l}\right)_{l}$. We employ Theorem 3.2 and obtain a function $v \in G S B D(\Omega)$ and a further not relabeled subsequence with $v^{l} \rightarrow v$ a.e in $\Omega$ and $e\left(\nabla v^{l}\right) \rightarrow e(\nabla v)$ weakly in $L^{2}\left(\Omega, \mathbb{R}_{\text {sym }}^{2 \times 2}\right)$.

We now select a suitable diagonal sequence such that (2.6)-(2.8) hold. First, we may suppose that after an infinitesimal modification we have $v_{k}^{l} \in W^{2, \infty}\left(\Omega \backslash \overline{J_{v_{k}^{l}}}\right)$ (see [16]). Consequently, by the coarea formula [3, Theorem 3.40] we get $\mathcal{H}^{1}\left(\partial^{*}\left\{\left|\nabla v_{k}^{l}\right| \leqq \lambda \varepsilon_{k}^{-1 / 8}\right\}\right)<\infty$ for all $\lambda \in\left(\frac{1}{2}, 1\right) \backslash H_{k}^{l}$, where $H_{k}^{l}$ is an $\mathcal{L}^{1}$-negligible set. Choosing $\lambda \in\left(\frac{1}{2}, 1\right) \backslash \bigcup_{k, l \in \mathbb{N}} H_{k}^{l}$ and defining $\hat{\phi}_{k}^{l}=$ $\chi_{\left[0, \lambda \varepsilon_{k}^{-1 / 8}\right]}\left(\left|\nabla v_{k}^{l}(x)\right|\right)$, the functions $\hat{v}_{k}^{l}:=\hat{\phi}_{k}^{l} v_{k}^{l}$ lie in $S B V(\Omega)$ by [3, Theorem 3.84]. Recalling the definition of $\phi_{k}^{l}$ before (4.9), we observe that by (4.9), (4.10) the functions fulfill $\left\|e\left(\nabla \hat{v}_{k}^{l}\right)\right\|_{L^{2}(\Omega)} \leqq C$ and $\left\|\nabla \hat{v}_{k}^{l}\right\|_{\infty} \leqq \varepsilon_{k}^{-1 / 8}$ for a constant independent of $k, l \in \mathbb{N}$. Moreover, by (4.4) we get $\hat{\phi}_{k}^{l} \rightarrow 1$ in measure on $\Omega$ as $k \rightarrow \infty$.

As weak convergence in $L^{2}$ is metrizable on bounded sets and convergence in measure is metrizable (take $\left.(f, g) \mapsto \int_{\Omega} \min \{|f-g|, 1\}\right)$, we can apply a diagonal sequence argument and find a not relabeled subsequence $\left(y_{n}\right)_{n}$ and a corresponding diagonal sequence $\left(w_{n}\right)_{n \in \mathbb{N}} \subset\left(\hat{v}_{k}^{l}\right)_{k, l}$ with corresponding partitions $\left(P_{j}^{n}\right)_{j}$ and piecewise rigid motions $\left(T_{n}\right)_{n}$ such that by (4.6), (4.7) and (4.11)

$w_{n} \rightarrow v$ in measure on $\Omega, \quad e\left(\nabla w_{n}\right) \rightarrow e(\nabla v)$ weakly in $L^{2}(\Omega)$,

$T_{n} \rightarrow T$ in $L^{2}(\Omega), \quad \nabla T_{n} \rightarrow \nabla T$ in $L^{2}(\Omega), \quad\left|P_{j}^{n} \triangle P_{j}\right| \rightarrow 0$ for all $j \in \mathbb{N}$

for $n \rightarrow \infty$. Up to a further subsequence we can assume $w_{n} \rightarrow v$ almost everywhere and $\nabla T_{n} \rightarrow \nabla T$ almost everywhere in $\Omega$. Finally, define $u_{n}=\nabla T_{n} w_{n}$ for all $n \in \mathbb{N}$ and let $u=\nabla T v$. Observe that (2.6)(ii), (2.7), and (2.8) hold. Moreover, as $\hat{\phi}_{k}^{l} \rightarrow 1$ in measure on $\Omega$ and $\left|\Omega \backslash \Omega_{k}^{l}\right| \rightarrow 0$ for $k, l \rightarrow \infty$, we also get (2.6)(i) recalling (4.2), (4.3) and possibly passing to a further subsequence.

To complete the proof of Theorem 2.2, it remains to show (2.9).

Proof of Theorem 2.2, part 2. To see (2.9)(i), it suffices to recall that each $\nabla T_{n}^{T} u_{n}$ coincides with some $\hat{\phi}_{k}^{l} v_{k}^{l}$ and thus Id $+\sqrt{\varepsilon_{n}} \nabla T_{n}^{T} \nabla u_{n}=\left(\nabla T_{k}^{l}\right)^{T} \nabla y_{k}^{l}$ almost everywhere on $\left\{\hat{\phi}_{k}^{l}=1\right\} \cap \Omega_{\rho_{l}}$ by (4.3). The assertion then follows from (3.11)(i) and the frame indifference of $W$. We now show (2.9)(ii). To this end, the estimate is first carried out in terms of the auxiliary functionals (see (3.8)). Afterwards, we conclude by passing to the limit $\rho \rightarrow 0$.

Let $v$ as given in (4.19) and recall $u=\nabla T v$. The sets $J_{v}^{d}:=\left\{x \in J_{v}\right.$ : $[v](x)=d\}$ for $d \in B_{1}(0) \backslash\{0\}$ are pairwise disjoint with $\mathcal{H}^{1}-\sigma$ finite union, that is $\mathcal{H}^{1}\left(J_{v}^{d}\right)=0$ up to at most countable values of $d$. Consequently, we can choose a sequence $\left(b_{j}\right)_{j}$ with $0<\left|b_{j}\right| \leqq 1$ such that $b_{i} \neq b_{j}$ and $\mathcal{H}^{1}\left(J_{v}^{b_{i}-b_{j}}\right)=0$ for $i \neq j$. Replacing $v$ by $\tilde{v}=v+\sum_{j} b_{j} \chi_{P_{j}}$, we thus obtain $\mathcal{H}^{1}\left(\left(\bigcup_{j} \partial^{*} P_{j} \cap \Omega\right) \backslash J_{\tilde{v}}\right)=0$. We first show that for (2.9)(ii) it suffices to prove

$$
\liminf _{k \rightarrow \infty} \mathcal{H}^{1}\left(J_{y_{k}}\right) \geqq \mathcal{H}^{1}\left(J_{\tilde{v}}\right) .
$$


Indeed, we get $\mathcal{H}^{1}\left(J_{\tilde{v}}\right)=\mathcal{H}^{1}\left(J_{u} \backslash \partial P\right)+\mathcal{H}^{1}(\partial P \cap \Omega)$, where for shorthand $\partial P=$ $\bigcup_{j} \partial^{*} P_{j}$ : We have $\mathcal{H}^{1}\left(J_{\tilde{v}}\right)=\mathcal{H}^{1}\left(J_{\tilde{v}} \cup(\partial P \cap \Omega)\right)=\mathcal{H}^{1}(\partial P \cap \Omega)+\mathcal{H}^{1}\left(J_{\tilde{v}} \backslash \partial P\right)$. Then it suffices to note $\mathcal{H}^{1}\left(J_{\tilde{v}} \backslash \partial P\right)=\mathcal{H}^{1}\left(J_{u} \backslash \partial P\right)$.

We now show (4.20) in two steps first passing to the limit $k \rightarrow \infty$ and then letting $l \rightarrow \infty$. We replace $v_{k}^{l}$ (see (4.3)) by $\tilde{v}_{k}^{l}=v_{k}^{l}+\sum_{j} b_{j} \chi_{P_{j}^{k, l}}$ and $v^{l}$ by $\tilde{v}^{l}=v^{l}+\sum_{j} b_{j} \chi_{P_{j}^{l}}$ noting that $\tilde{v}_{k}^{l} \rightarrow \tilde{v}^{l}$ for $k \rightarrow \infty$ (cf. (4.6)) and $\tilde{v}^{l} \rightarrow \tilde{v}$ for $l \rightarrow \infty$ in the sense of (3.4). In the following we write $J_{k}^{l}=J_{\tilde{v}_{k}^{l}} \cap \Omega_{\rho_{l}}$ and $\partial P^{k, l}:=\bigcup_{j} \partial^{*} P_{j}^{k, l}$ for shorthand, where $\Omega_{\rho_{l}}$ was defined before (3.8). We obtain by (4.3), (3.11)(ii) and Theorem 3.5

$$
\begin{aligned}
\mathcal{H}^{1}\left(J_{y_{k}}\right)+C \rho_{l} & \geqq \int_{J_{y_{k} l} \backslash \partial P^{k, l}} f_{\varepsilon_{k}}^{\rho_{l}}\left(\left|\left[y_{k}^{l}\right]\right|\right) \mathrm{d} \mathcal{H}^{1}+\mathcal{H}^{1}\left(\partial P^{k, l} \cap \Omega_{\rho_{l}}\right) \\
& \geqq \int_{J_{k}^{l} \backslash \partial P^{k, l}} \theta_{\rho_{l}}\left(\left|\left[\tilde{v}_{k}^{l}\right]\right|\right) \mathrm{d} \mathcal{H}^{1}+\mathcal{H}^{1}\left(\partial P^{k, l} \cap \Omega_{\rho_{l}}\right) \geqq \int_{J_{k}^{l}} \theta_{\rho_{l}}\left(\left|\left[\tilde{v}_{k}^{l}\right]\right|\right) \mathrm{d} \mathcal{H}^{1},
\end{aligned}
$$

where $\theta_{\sigma}(t):=\min \left\{\frac{t}{\sigma}, 1\right\}$ for $\sigma>0$. We cannot directly apply lower semicontinuity results for $G S B D$ functions due to the involved function $\theta_{\rho_{l}}$. We therefore pass to the limit $k \rightarrow \infty$ on one-dimensional sections.

Recall the measure $\hat{\mu}_{\tilde{v}^{l}}^{\sigma, \xi}$ defined in (3.5) for $\sigma \geqq 0$. By Lemma 3.3 we have

$$
\hat{\mu}_{\tilde{v}^{l}}^{\sigma, \xi}(U) \leqq \liminf _{k \rightarrow \infty} \hat{\mu}_{\tilde{v}_{k}^{l}}^{\sigma, \xi}(U)
$$

for all $\sigma \geqq 0, \xi \in S^{1}$ and for every open set $U \subset \Omega$. Let $\kappa_{1}=\int_{S^{1}}|v \cdot \xi| \mathrm{d} \mathcal{H}^{1}(\xi)$ for some $v \in S^{1}$ which clearly does not depend on the particular choice of $v$. Using Fatou's lemma and (3.6) we compute

$$
\begin{aligned}
\liminf _{k \rightarrow \infty} \mathcal{H}^{1}\left(J_{y_{k}}\right) & +C \rho_{l} \geqq \liminf _{k \rightarrow \infty} \int_{J_{k}^{l}} \theta_{\sigma}\left(\left|\left[\tilde{v}_{k}^{l}\right]\right|\right) \mathrm{d} \mathcal{H}^{1} \\
& \geqq \kappa_{1}^{-1} \int_{S^{1}} \liminf _{k \rightarrow \infty} \int_{J_{k}^{l}} \theta_{\sigma}\left(\left|\left[\tilde{v}_{k}^{l}\right](x)\right|\right)\left|\nu_{\tilde{v}_{k}^{l}}(x) \cdot \xi\right| \mathrm{d} \mathcal{H}^{1}(x) \mathrm{d} \mathcal{H}^{1}(\xi) \\
& \geqq \kappa_{1}^{-1} \int_{S^{1}} \liminf _{k \rightarrow \infty} \hat{\mu}_{\tilde{v}_{k}^{l}}^{\sigma, \xi}\left(\Omega_{\rho_{l}}\right) \mathrm{d} \mathcal{H}^{1}(\xi) \geqq \kappa_{1}^{-1} \int_{S^{1}} \hat{\mu}_{\tilde{v}^{l}}^{\sigma, \xi}\left(\Omega_{\rho_{l}}\right) \mathrm{d} \mathcal{H}^{1}(\xi) .
\end{aligned}
$$

We pass to the limit $l \rightarrow \infty$ (that is $\rho_{l} \rightarrow 0$ ) and obtain by Lemma 3.3 and the dominated convergence theorem

$$
\liminf _{k \rightarrow \infty} \mathcal{H}^{1}\left(J_{y_{k}}\right) \geqq \kappa_{1}^{-1} \int_{S^{1}} \hat{\mu}_{\tilde{v}}^{\sigma, \xi}(\Omega) \mathrm{d} \mathcal{H}^{1}(\xi) .
$$

Recall that $\theta_{\sigma} \rightarrow 1$ pointwise for $\sigma \rightarrow 0$. Now letting $\sigma \rightarrow 0$ we obtain by the dominated convergence theorem and (3.6)

$$
\begin{aligned}
\liminf _{k \rightarrow \infty} \mathcal{H}^{1}\left(J_{y_{k}}\right) & \geqq \kappa_{1}^{-1} \int_{S^{1}} \hat{\mu}_{\tilde{v}}^{0, \xi}(\Omega) \mathrm{d} \mathcal{H}^{1}(\xi) \\
& =\kappa_{1}^{-1} \int_{S^{1}} \int_{J_{\tilde{v}}^{\xi}}\left|\nu_{\tilde{v}}(x) \cdot \xi\right| \mathrm{d} \mathcal{H}^{1}(x) \mathrm{d} \mathcal{H}^{1}(\xi)=\mathcal{H}^{1}\left(J_{\tilde{v}}\right) .
\end{aligned}
$$

This gives (4.20) and completes the proof. 
Remark 4.3. Using (2.6)(ii), (2.9)(i) and arguing as in (4.9), (4.10), we observe that all sequences $\left(u_{k}, \mathcal{P}_{k}, T_{k}\right)$ in Definition 2.3 satisfy $\left\|e\left(\nabla T_{k}^{T} \nabla u_{k}\right)\right\|_{L^{2}(\Omega)} \leqq C$ for $C>0$ only depending on $\sup _{k} E_{\varepsilon_{k}}\left(y_{k}\right), \Omega$, and the constant in (2.2).

At the end of this section we briefly note that our compactness result provides an alternative proof of the piecewise rigidity result given in Theorem 3.9 (at least in a planar setting).

Proof of Theorem 3.9 for $d=2$. Let $y \in S B V(\Omega)$ with $\mathcal{H}^{1}\left(J_{y}\right)<\infty$ as well as $\int_{\Omega} \operatorname{dist}^{2}(\nabla y, S O(2))=0$ be given. First, assume $y \in L^{\infty}(\Omega)$. Define an arbitrary infinitesimal sequence $\left(\varepsilon_{k}\right)_{k}$ and the sequence $y_{k}=y$ for all $k \in \mathbb{N}$. Applying Theorem 2.2 we obtain piecewise rigid motions $T, T_{k}$ such that $T_{k} \rightarrow T, \nabla T_{k} \rightarrow$ $\nabla T$ in $L^{2}(\Omega)$ by (2.7) up to passing to a subsequence. Moreover, $y_{k}-T_{k} \rightarrow 0$ almost everywhere in $\Omega$ for $k \rightarrow \infty$ by (2.6)(i). This implies $y=T$ is a piecewise rigid motion. If $y \notin L^{\infty}(\Omega)$, using the $B V$ coarea formula we can approximate $y$ by a sequence $y \chi_{\Phi_{k}}+\mathbf{i d} \chi_{\Omega \backslash \Phi_{k}} \in S B V(\Omega) \cap L^{\infty}(\Omega)$ with $\sup _{k} \mathcal{H}^{1}\left(\partial^{*} \Phi_{k}\right)<\infty$, $\left|\Phi_{k}\right| \rightarrow 0$ for $k \rightarrow \infty$ and conclude by Theorem 3.7.

\section{Admissible and Coarsest Partitions and Limiting Configurations}

In this section we will prove Theorem 2.6. Let $\left(y_{k}\right)_{k}$ be a (sub-)sequence as considered in Theorem 2.2. Recall Definition 2.5. For notational convenience we will drop the dependence of $\left(y_{k}\right)_{k}$ in the sets $\mathcal{Z}_{P}, \mathcal{Z}_{u}, \mathcal{Z}_{T}$. We introduce a partial order on the admissible partitions $\mathcal{Z}_{P}$ : Given two partitions $\mathcal{P}^{1}:=\left(P_{j}^{1}\right)_{j}, \mathcal{P}^{2}:=$ $\left(P_{j}^{2}\right)_{j}$ in $\mathcal{Z}_{P}$ we say $\mathcal{P}^{2} \geqq \mathcal{P}^{1}$ if

$$
\text { for all } P_{j_{1}}^{1} \text { there exists } P_{j_{2}}^{2} \text { such that }\left|P_{j_{1}}^{1} \backslash P_{j_{2}}^{2}\right|=0 \text {. }
$$

Note that Theorem 3.5 implies $\bigcup_{j} \partial^{*} P_{j}^{1} \supset \bigcup_{j} \partial^{*} P_{j}^{2}$ up to an $\mathcal{H}^{1}$-negligible set. We observe that if $\mathcal{P}^{1} \geqq \mathcal{P}^{2}$ and $\mathcal{P}^{2} \geqq \mathcal{P}^{1}$, abbreviated by $\mathcal{P}^{1}=\mathcal{P}^{2}$ hereafter, then the Caccioppoli partitions coincide: After a possible reordering of the sets we find $\left|P_{j}^{1} \triangle P_{j}^{2}\right|=0$ for all $j \in \mathbb{N}$.

We begin with the observation that the piecewise rigid motion is uniquely determined in the limit.

Lemma 5.1. Let $\left(y_{k}\right)_{k}$ be a (sub-)sequence as considered in Theorem 2.2. Then there is a unique $T \in \mathcal{Z}_{T}$.

Proof. Assume there are $T, \hat{T} \in \mathcal{Z}_{T}$. Let $(u, \mathcal{P}, T),(\hat{u}, \hat{\mathcal{P}}, \hat{T}) \in \mathcal{D}_{\infty}$ according to Definition 2.5(ii) and let $\left(u_{k}, \mathcal{P}_{k}, T_{k}\right),\left(\hat{u}_{k}, \hat{\mathcal{P}}_{k}, \hat{T}_{k}\right) \in \mathcal{D}$ for $k \in \mathbb{N}$ be triples given by Definition 2.3. As $u_{k}-\hat{u}_{k}-\left(\varepsilon_{k}^{-1 / 2}\left(T_{k}-\hat{T}_{k}\right)\right) \rightarrow 0$ almost everywhere by (2.6)(i) and $u_{k}-\hat{u}_{k}$ converges pointwise almost everywhere (and the limits lie in $\mathbb{R}$ almost everywhere) by (2.8)(i), we get $T_{k}-\hat{T}_{k} \rightarrow 0$ pointwise almost everywhere. By (2.7)(ii) this implies $T=\hat{T}$.

From now on $T$ will always stand for the rigid motion given by Lemma 5.1. 


\subsection{Equivalent Characterization of the Coarsest Partition}

We state a lemma giving an equivalent characterization of the coarsest partition (recall Definition 2.5(iv)).

Lemma 5.2. Let $\left(y_{k}\right)_{k}$ be a (sub-)sequence as considered in Theorem 2.2. Then $\mathcal{P} \in \mathcal{Z}_{P}$ is coarsest if and only if it is a maximal element in the partial order $\left(\mathcal{Z}_{P}, \geqq\right)$, that is $\hat{\mathcal{P}} \geqq \mathcal{P}$ implies $\hat{\mathcal{P}}=\mathcal{P}$.

Proof. (1) Assume that $\mathcal{P}=\left(P_{j}\right)_{j}$ was not coarsest. According to Definition 2.5(iv) let $u$ and $\left(u_{k}, \mathcal{P}_{k}, T_{k}\right) \in \mathcal{D}$ be given such that $(u, \mathcal{P}, T) \in \mathcal{D}_{\infty}$ and (2.6)(2.9) hold. Without restriction, possibly passing to a subsequence and reordering the partition, we assume that $\varepsilon_{k}^{-1 / 2}\left(\left|R_{1}^{k}-R_{2}^{k}\right|+\left|b_{1}^{k}-b_{2}^{k}\right|\right) \leqq C$ for all $k \in \mathbb{N}$ (cf. (2.10)). By (3.12) we obtain $A^{k} \in \mathbb{R}_{\text {skew }}^{2 \times 2}$ for $k \in \mathbb{N}$ with $\left|A^{k}\right| \leqq C$ such that $R_{2}^{k}-R_{1}^{k}=R_{1}^{k}\left(\left(R_{1}^{k}\right)^{T} R_{2}^{k}-\mathbf{I d}\right)=R_{1}^{k}\left(\sqrt{\varepsilon_{k}} A^{k}+O\left(\varepsilon_{k}\right)\right)$. Passing to a (not relabeled) subsequence we then obtain for all $x \in \Omega$

$$
\begin{aligned}
S(x) & :=\lim _{k \rightarrow \infty} \frac{1}{\sqrt{\varepsilon_{k}}}\left(\left(R_{2}^{k}-R_{1}^{k}\right) x+b_{2}^{k}-b_{1}^{k}\right) \\
& =\lim _{k \rightarrow \infty} \frac{1}{\sqrt{\varepsilon_{k}}}\left(\sqrt{\varepsilon_{k}} R_{1}^{k} A^{k} x+b_{2}^{k}-b_{1}^{k}\right)+O\left(\sqrt{\varepsilon_{k}}\right)=R A x+b
\end{aligned}
$$

for some $A \in \mathbb{R}_{\text {skew }}^{2 \times 2}, b \in \mathbb{R}^{2}$ and $R=\lim _{k \rightarrow \infty} R_{1}^{k}$. We now introduce $\hat{\mathcal{P}}_{k}, \hat{\mathcal{P}}, \hat{T}_{k}$, $\hat{u}_{k}, \hat{u}$ as follows. Let $\hat{P}_{1}^{k}=P_{1}^{k} \cup P_{2}^{k}, \hat{P}_{2}^{k}=\emptyset, \hat{P}_{j}^{k}=P_{j}^{k}$ for $j \geqq 3$ and likewise for the limiting partition $\hat{\mathcal{P}}$. Let $\hat{T}_{k}(x)=R_{1}^{k} x+b_{1}^{k}$ for $x \in \hat{P}_{1}^{k}$ and $\hat{T}_{k}(x)=T_{k}(x)$ for $x \in \Omega \backslash \hat{P}_{1}^{k}$. Furthermore, we let

$$
\hat{u}_{k}=u_{k}+\frac{1}{\sqrt{\varepsilon_{k}}}\left(\left(R_{2}^{k}-R_{1}^{k}\right) \cdot+b_{2}^{k}-b_{1}^{k}\right) \chi_{P_{2}^{k}}
$$

and $\hat{u}=u+(R A \cdot+b) \chi_{P_{2}}($ see $(5.2))$. We now show that $\left(\hat{u}_{k}, \hat{\mathcal{P}}_{k}, \hat{T}_{k}\right)$ converges to $(\hat{u}, \hat{\mathcal{P}}, T)$ in the sense of (2.6)-(2.9). First, (2.6)(i) clearly holds since $\hat{T}_{k}-T_{k}=$ $\left(\left(R_{1}^{k}-R_{2}^{k}\right) \cdot+b_{1}^{k}-b_{2}^{k}\right) \chi_{P_{2}^{k}}$. Moreover, we derive that (2.7) holds as $\left|R_{1}^{k}-R_{2}^{k}\right|+$ $\left|b_{1}^{k}-b_{2}^{k}\right| \rightarrow 0$ for $k \rightarrow \infty$. Since $\mathcal{H}^{1}\left(J_{u} \backslash \bigcup_{j} \partial^{*} P_{j}\right)+\mathcal{H}^{1}\left(\bigcup_{j} \partial^{*} P_{j} \cap \Omega\right) \geqq$ $\mathcal{H}^{1}\left(J_{\hat{u}} \backslash \bigcup_{j} \partial^{*} \hat{P}_{j}\right)+\mathcal{H}^{1}\left(\bigcup_{j} \partial^{*} \hat{P}_{j} \cap \Omega\right)$, also (2.9)(ii) is satisfied.

As $\left|R_{2}^{k}-R_{1}^{k}\right| \leqq C \sqrt{\varepsilon_{k}}$, we note that $\left\|\nabla \hat{u}_{k}\right\|_{L^{\infty}(\Omega)} \leqq c \varepsilon_{k}^{-1 / 8}$ for $c>0$ large enough and thus (2.6)(ii) holds. It remains to verify (2.8) and (2.9)(i). First, (2.8)(i) follows from (5.2) and the definition of $\hat{u}$. We use $R_{2}^{k}=R_{1}^{k}+\sqrt{\varepsilon_{k}} R_{1}^{k} A^{k}+O\left(\varepsilon_{k}\right)$, $\left|A^{k}\right| \leqq C$ and $\left\|\nabla u_{k}\right\|_{L^{\infty}(\Omega)} \leqq c \varepsilon_{k}^{-1 / 8}$ to find almost everywhere on $P_{2}^{k}$

$$
\nabla \hat{T}_{k}^{T} \nabla \hat{u}_{k}=\left(R_{1}^{k}\right)^{T} \nabla u_{k}+A^{k}+O\left(\sqrt{\varepsilon_{k}}\right)=\left(R_{2}^{k}\right)^{T} \nabla u_{k}+A^{k}+O\left(\varepsilon_{k}^{3 / 8}\right) .
$$

Now we get

$$
\begin{aligned}
\chi_{\hat{P}_{1}^{k}} e\left(\nabla \hat{T}_{k}^{T} \nabla \hat{u}_{k}\right) & =\sum_{j=1,2} \chi_{P_{j}^{k}} e\left(\left(R_{j}^{k}\right)^{T} \nabla u_{k}\right)+\chi_{P_{2}^{k}} e\left(A^{k}\right)+O\left(\varepsilon_{k}^{3 / 8}\right) \\
& \rightarrow \sum_{j=1,2} \chi_{P_{j}} e\left(R_{j}^{T} \nabla u\right)=\chi_{\hat{P}_{1}} e\left(R^{T} \nabla \hat{u}\right)=\chi_{\hat{P}_{1}} e\left(\nabla T^{T} \nabla \hat{u}\right)
\end{aligned}
$$


weakly in $L^{2}\left(\Omega, \mathbb{R}_{\mathrm{sym}}^{2 \times 2}\right)$. This gives (2.8)(ii). By the assumptions on $W$, a Taylor expansion yields $W(G)=\frac{1}{2} Q(e(G-\mathbf{I d}))+\omega_{\mathrm{W}}(G-\mathbf{I d})$ for $G \in \mathbb{R}^{2 \times 2}$, where $\sup \left\{|F|^{-3} \omega_{W}(F):|F| \leqq 1\right\} \leqq C$ and $Q=D^{2} W($ Id). Thus, we obtain by (5.3) and $\left\|\nabla u_{k}\right\|_{L^{\infty}(\Omega)} \leqq c \varepsilon_{k}^{-\overline{1 /} / 8}$

$$
\begin{aligned}
\frac{1}{\varepsilon_{k}} \int_{P_{2}^{k}} W\left(\mathbf{I d}+\sqrt{\varepsilon_{k}} \nabla \hat{T}_{k}^{T} \nabla \hat{u}_{k}\right) & =\int_{P_{2}^{k}}\left(\frac{1}{2} Q\left(e\left(\nabla \hat{T}_{k}^{T} \nabla \hat{u}_{k}\right)\right)+\frac{1}{\varepsilon_{k}} \omega_{W}\left(\sqrt{\varepsilon_{k}} \nabla \hat{T}_{k}^{T} \nabla \hat{u}_{k}\right)\right) \\
& =\int_{P_{2}^{k}}\left(\frac{1}{2} Q\left(e\left(\nabla T_{k}^{T} \nabla u_{k}\right)\right)+\frac{\omega_{W}\left(\sqrt{\varepsilon_{k}} \nabla \hat{u}_{k}\right)}{\varepsilon_{k}}\right)+O\left(\varepsilon_{k}^{\frac{1}{4}}\right)
\end{aligned}
$$

and likewise

$\frac{1}{\varepsilon_{k}} \int_{P_{2}^{k}} W\left(\mathbf{I d}+\sqrt{\varepsilon_{k}} \nabla T_{k}^{T} \nabla u_{k}\right)=\int_{P_{2}^{k}}\left(\frac{1}{2} Q\left(e\left(\nabla T_{k}^{T} \nabla u_{k}\right)\right)+\frac{1}{\varepsilon_{k}} \omega_{W}\left(\sqrt{\varepsilon_{k}} \nabla u_{k}\right)\right)$.

In both estimates the second terms converge to 0 using $\left\|\nabla u_{k}\right\|_{\infty}+\left\|\nabla \hat{u}_{k}\right\|_{\infty} \leqq$ $c \varepsilon_{k}^{-1 / 8}$ and arguing as in (4.10). Consequently, we get

$$
\frac{1}{\varepsilon_{k}} \int_{P_{2}^{k}} W\left(\mathbf{I d}+\sqrt{\varepsilon_{k}} \nabla \hat{T}_{k}^{T} \nabla \hat{u}_{k}\right)=\frac{1}{\varepsilon_{k}} \int_{P_{2}^{k}} W\left(\mathbf{I d}+\sqrt{\varepsilon_{k}} \nabla T_{k}^{T} \nabla u_{k}\right)+o(1)
$$

for $\varepsilon_{k} \rightarrow 0$, that is (2.9)(i) holds. Therefore, $\hat{\mathcal{P}}$ is an admissible partition and thus $\mathcal{P}$ is not maximal.

(2) Conversely, assume that $\mathcal{P}=\left(P_{j}\right)_{j}$ was not maximal, that is we find $\hat{\mathcal{P}}=\left(\hat{P}_{j}\right)_{j}$ with $\hat{\mathcal{P}} \geqq \mathcal{P}, \hat{\mathcal{P}} \neq \mathcal{P}$. Upon reordering we may assume that $P_{1} \cap \hat{P}_{1}$ and $P_{2} \cap \hat{P}_{1}$ have positive $\mathcal{L}^{2}$-measure. According to Definition 2.5(i) let $u, \hat{u}$ and $\left(u_{k}, \mathcal{P}_{k}, T_{k}\right),\left(\hat{u}_{k}, \hat{\mathcal{P}}_{k}, \hat{T}_{k}\right) \in \mathcal{D}$ be given such that $(u, \mathcal{P}, T),(\hat{u}, \hat{\mathcal{P}}, T) \in \mathcal{D}_{\infty}$ and (2.6)-(2.9) hold. As by (2.8)(i) $u_{k}$ and $\hat{u}_{k}$ convergence pointwise almost everywhere, by (2.6) also $\varepsilon_{k}^{-1 / 2}\left(T_{k}-\hat{T}_{k}\right)$ converges pointwise almost everywhere (and the limits lie in $\mathbb{R}$ almost everywhere). But this implies $\varepsilon_{k}^{-1 / 2}\left(\left|R_{j}^{k}-\hat{R}_{1}^{k}\right|+\left|b_{j}^{k}-\hat{b}_{1}^{k}\right|\right) \leqq C$ for $j=1,2$ and $k \in \mathbb{N}$. Then $\varepsilon_{k}^{-1 / 2}\left(\left|R_{1}^{k}-R_{2}^{k}\right|+\left|b_{1}^{k}-b_{2}^{k}\right|\right) \leqq C$ by the triangle inequality. Thus, (2.10) is violated and $\mathcal{P}$ is not a coarsest partition.

The alternative characterization now directly implies that there is at most one coarsest partition.

Lemma 5.3. Let $\left(y_{k}\right)_{k}$ be a (sub-)sequence as considered in Theorem 2.2. Then there is at most one maximal element in $\left(\mathcal{Z}_{P}, \geqq\right)$.

Proof. Assume there are two maximal elements $\mathcal{P}^{1}=\left(P_{j}^{1}\right)_{j}, \mathcal{P}^{2}=\left(P_{j}^{2}\right)_{j} \in \mathcal{Z}_{P}$ with $\mathcal{P}^{1} \neq \mathcal{P}^{2}$. As before, without restriction we may assume that $P_{1}^{1} \cap P_{1}^{2}$ and $P_{2}^{1} \cap P_{1}^{2}$ have positive $\mathcal{L}^{2}$-measure. We proceed as in the proof of Lemma 5.2(2) to see that $\mathcal{P}^{1}$ is not coarsest and thus not a maximal element in $\left(\mathcal{Z}_{P}, \geqq\right)$. 


\subsection{Admissible Configurations}

We now analyze the admissible configurations if the partitions are given. Recall the definition of the set of piecewise infinitesimal rigid motions $\mathcal{A}(\mathcal{P})$ below (2.4).

Lemma 5.4. Let $\left(y_{k}\right)_{k}$ be a (sub-)sequence as considered in Theorem 2.2 and let $T \in \mathcal{Z}_{T}$ be the unique mapping given by Lemma 5.1. Let $\mathcal{P}, \hat{\mathcal{P}} \in \mathcal{Z}_{P}$ such that $\hat{\mathcal{P}} \geqq \mathcal{P}$ and $\hat{u} \in \mathcal{Z}_{u}(\hat{\mathcal{P}})$. Then $\mathcal{Z}_{u}(\mathcal{P})=\hat{u}+\nabla T \mathcal{A}(\mathcal{P})$.

Proof. (1) To see $\mathcal{Z}_{u}(\mathcal{P}) \subset \hat{u}+\nabla T \mathcal{A}(\mathcal{P})$, we have to show that $u-\hat{u} \in \nabla T \mathcal{A}(\mathcal{P})$ for all $u \in \mathcal{Z}_{u}(\mathcal{P})$. To this end, consider $P_{j} \in \mathcal{P}, \hat{P}_{i} \in \hat{\mathcal{P}}$ such that $\left|P_{j} \backslash \hat{P}_{i}\right|=0$. Let $\left(u_{k}, \mathcal{P}_{k}, T_{k}\right),\left(\hat{u}_{k}, \hat{\mathcal{P}}_{k}, \hat{T}_{k}\right) \in \mathcal{D}$ be given according to Definition 2.3. As $u_{k}-\hat{u}_{k}$ and thus $\varepsilon_{k}^{-1 / 2}\left(T_{k}-\hat{T}_{k}\right)$ converge pointwise almost everywhere by (2.6)(i), (2.8)(i), we find $\left|R_{j}^{k}-\hat{R}_{i}^{k}\right|+\left|b_{j}^{k}-\hat{b}_{i}^{k}\right| \leqq C \sqrt{\varepsilon_{k}}$. Repeating the argument in (5.2) we find some $A \in \mathbb{R}_{\text {skew }}^{2 \times 2}, b \in \mathbb{R}^{2}$ such that for almost every $x \in P_{j}$

$$
\begin{aligned}
u(x)-\hat{u}(x)= & \lim _{k \rightarrow \infty} u_{k}(x)-\hat{u}_{k}(x)=\lim _{k \rightarrow \infty} \varepsilon_{k}^{-1 / 2}\left(\hat{T}_{k}(x)\right. \\
& \left.-T_{k}(x)\right)=\nabla T(x)(A x+b) .
\end{aligned}
$$

(2) Conversely, to see $\mathcal{Z}_{u}(\mathcal{P}) \supset \hat{u}+\nabla T \mathcal{A}(\mathcal{P})$ we first consider the special case $\mathcal{P}=\hat{\mathcal{P}}=\left(P_{j}\right)_{j}$. Let $\bar{u} \in \mathcal{Z}_{u}(\mathcal{P})$ and $\bar{A}=\sum_{j}\left(A_{j} \cdot+d_{j}\right) \chi_{P_{j}} \in \mathcal{A}(\mathcal{P})$ be given. We have to show that $u:=\bar{u}+\nabla T \bar{A} \in \mathcal{Z}_{u}(\mathcal{P})$.

According to Definition 2.5(iii) let $\left(\bar{u}_{k}, \mathcal{P}_{k}, \bar{T}_{k}\right) \in \mathcal{D}$ be given such that (2.6)(2.9) hold with the limiting triple $(\bar{u}, \mathcal{P}, T)$. Assume that $\bar{T}_{k}$ has the form $\bar{T}_{k}=$ $\sum_{j}\left(\bar{R}_{j}^{k} \cdot+\bar{b}_{j}^{k}\right) \chi_{P_{j}^{k}}$. Now choose $R_{j}^{k}$ such that $\left|R_{j}^{k}-\bar{R}_{j}^{k}\left(\mathbf{I d}-\sqrt{\varepsilon_{k}} A_{j}\right)\right|=\operatorname{dist}\left(\bar{R}_{j}^{k}(\mathbf{I d}-\right.$ $\left.\left.\sqrt{\varepsilon_{k}} A_{j}\right), S O(2)\right)$ and let $b_{j}^{k}=\bar{b}_{j}^{k}-\sqrt{\varepsilon_{k}} \bar{R}_{j}^{k} d_{j}$. By (3.12) we have

$$
R_{j}^{k}=\bar{R}_{j}^{k}-\sqrt{\varepsilon_{k}} \bar{R}_{j}^{k} A_{j}-\omega_{j, k} \text { with }\left|\omega_{j, k}\right| \leqq C \varepsilon_{k}\left|A_{j}\right|^{2} \text { for all } j \in \mathbb{N} .
$$

Let $I_{k}=\left\{j \in \mathbb{N}:\left|A_{j}\right|+\left|d_{j}\right| \leqq \varepsilon_{k}^{-1 / 8}\right\}$ and $V_{k}=\bigcup_{j \in \mathbb{N} \backslash I_{k}} P_{j}^{k}$. Note that $\left|V_{k}\right| \rightarrow 0$ for $k \rightarrow \infty$ and $\left|\varepsilon_{k}^{-1 / 2} \omega_{j, k}\right| \leqq C \varepsilon_{k}^{1 / 4}$ for $j \in I_{k}$. Define

$$
T_{k}=\sum_{j \in I_{k}}\left(R_{j}^{k} \cdot+b_{j}^{k}\right) \chi_{P_{j}^{k}}+\bar{T}_{k} \chi_{V_{k}}, \quad u_{k}=\bar{u}_{k}+\frac{1}{\sqrt{\varepsilon_{k}}}\left(\bar{T}_{k}-T_{k}\right) .
$$

We now show that $\left(u_{k}, \mathcal{P}_{k}, T_{k}\right)$ converges to $(u, \mathcal{P}, T)$ in the sense of (2.6)-(2.9). First, (2.6)(i) clearly holds. Moreover, $\mathcal{H}^{1}\left(J_{u} \backslash \bigcup_{j} \partial^{*} P_{j}\right)=\mathcal{H}^{1}\left(J_{\bar{u}} \backslash \bigcup_{j} \partial^{*} P_{j}\right)$ and thus (2.9)(ii) is satisfied. By (5.5) we find

$$
T_{k}=\bar{T}_{k}-\sum_{j \in I_{k}}\left(\sqrt{\varepsilon_{k}} \bar{R}_{j}^{k} A_{j} \cdot+\omega_{j, k} \cdot+\sqrt{\varepsilon_{k}} \bar{R}_{j}^{k} d_{j}\right) \chi_{P_{j}^{k}} \rightarrow T
$$

in measure for $k \rightarrow \infty$. Then it is not hard to see that $T_{k} \rightarrow T$ and $\nabla T_{k} \rightarrow \nabla T$ in $L^{2}(\Omega)$ which gives (2.7). Likewise, we obtain

$$
\begin{aligned}
u_{k}-\bar{u}_{k} & =\frac{1}{\sqrt{\varepsilon_{k}}}\left(\bar{T}_{k}-T_{k}\right)=\sum_{j \in I_{k}}\left(\bar{R}_{j}^{k}\left(A_{j} \cdot+d_{j}\right)+\frac{1}{\sqrt{\varepsilon_{k}}} \omega_{j, k} \cdot\right) \chi_{P_{j}^{k}} \\
& \rightarrow \nabla T \sum_{j}\left(A_{j} \cdot+d_{j}\right) \chi_{P_{j}}=\nabla T \bar{A}
\end{aligned}
$$


pointwise almost everywhere which implies $u_{k} \rightarrow \bar{u}+\nabla T \bar{A}$ and shows (2.8)(i). By the definition of $V_{k}$ we have

$$
\left\|\nabla u_{k}-\nabla \bar{u}_{k}\right\|_{L^{\infty}(\Omega)} \leqq\left\|\sum_{j} \chi_{P_{j}^{k}}\left(\bar{R}_{j}^{k} A_{j}+\varepsilon_{k}^{-1 / 2} \omega_{j, k}\right)\right\|_{L^{\infty}\left(\Omega \backslash V_{k}\right)} \leqq C \varepsilon_{k}^{-1 / 8} .
$$

Therefore, $\left\|\nabla u_{k}\right\|_{\infty} \leqq c \varepsilon_{k}^{-1 / 8}$ for $c$ large enough, which shows (2.6)(ii). Arguing as in (5.3) we get by $\left\|\nabla \bar{u}_{k}\right\|_{L^{\infty}(\Omega)} \leqq c \varepsilon_{k}^{-1 / 8}$ and (5.5)

$$
\begin{aligned}
\left(R_{j}^{k}\right)^{T} \nabla u_{k}(x) & =\left(R_{j}^{k}\right)^{T} \nabla \bar{u}_{k}(x)+\left(R_{j}^{k}\right)^{T} \bar{R}_{j}^{k} A_{j}+\left(R_{j}^{k}\right)^{T} \varepsilon_{k}^{-1 / 2} w_{j, k} \\
& =\left(\bar{R}_{j}^{k}\right)^{T} \nabla \bar{u}_{k}(x)+A_{j}+O\left(\varepsilon_{k}^{1 / 4}\right)
\end{aligned}
$$

for almost every $x \in P_{j}^{k}, j \in I_{k}$. Thus, (2.8)(ii) follows from the fact that (2.8)(ii) holds for the sequence $\bar{u}_{k}$ and

$$
\sum_{j \in I_{k}} \int_{P_{j}^{k}}\left|e\left(\left(R_{j}^{k}\right)^{T} \nabla u_{k}\right)-e\left(\left(\bar{R}_{j}^{k}\right)^{T} \nabla \bar{u}_{k}\right)\right|^{2} \leqq C \varepsilon_{k}^{1 / 2} \rightarrow 0 .
$$

Finally, the above estimates together with a similar argumentation as in (5.4) yield (2.9)(i).

In the general case we have to show $u:=\hat{u}+\nabla T \bar{A} \in \mathcal{Z}_{u}(\mathcal{P})$ for given $\hat{u} \in \mathcal{Z}_{u}(\hat{\mathcal{P}}), \hat{\mathcal{P}} \geqq \mathcal{P}$, and $\bar{A} \in \mathcal{A}(\mathcal{P})$. As $\mathcal{P} \in \mathcal{Z}_{P}$, we find some $\bar{u} \in \mathcal{Z}_{u}(\mathcal{P})$ which by (1) satisfies $\bar{u}-\hat{u}=\nabla T \hat{A}$ for some $\hat{A} \in \mathcal{A}(\mathcal{P})$. Thus, we get $u=\bar{u}+\nabla T(\bar{A}-\hat{A})$ and by the special case in (2) we know that $u \in \mathcal{Z}_{u}(\mathcal{P})$, as desired.

\subsection{Existence of Coarsest Partitions}

To guarantee the existence of coarsest partitions we show that each totally ordered subset has upper bounds such that afterwards we may apply Zorn's lemma.

Lemma 5.5. Let $\left(y_{k}\right)_{k}$ be a (sub-)sequence as considered in Theorem 2.2. Let I be an arbitrary index set and let $\left\{\mathcal{P}_{i}=\left(P_{i, j}\right)_{j}: i \in I\right\} \subset \mathcal{Z}_{P}$ be a totally ordered subset, that is for each $i_{1}, i_{2} \in I$ we have $\mathcal{P}_{i_{1}} \leqq \mathcal{P}_{i_{2}}$ or $\mathcal{P}_{i_{2}} \leqq \mathcal{P}_{i_{1}}$. Then there is a partition $\mathcal{P} \in \mathcal{Z}_{P}$ with $\mathcal{P}_{i} \leqq \mathcal{P}$ for all $i \in I$.

Proof. Step I: To prove the existence of an upper bound we first show that it suffices to consider a suitable countable subset of $\left\{\mathcal{P}_{i}: i \in I\right\}$. For notational convenience we write $i_{1} \leqq i_{2}$ for $i_{1}, i_{2} \in I$ if $\mathcal{P}_{i_{1}} \leqq \mathcal{P}_{i_{2}}$. Choose an arbitrary $i_{0} \in I$ and note that it suffices to find an upper bound for all $i \geqq i_{0}$. For each $k \in \mathbb{N}$ we introduce partitions $\mathcal{P}_{i}^{k}=\left(P_{i, j}^{k}\right)_{j \geqq 0}$ consisting of the components $P_{i, j}^{k}=P_{i, j} \backslash \bigcup_{l \geqq k} P_{i_{0}, l}$ for $j \in \mathbb{N}$ and $P_{i, 0}^{k}=\bigcup_{l \geqq k} P_{i_{0}, l}$. (Note that the partitions $\mathcal{P}_{i}^{k}$ are possibly not ordered.) By (5.1) we get that $\mathcal{P}_{i_{1}}^{k} \leqq \mathcal{P}_{i_{2}}^{k}$ if $i_{0} \leqq i_{1} \leqq i_{2}$. Typically, $\mathcal{P}_{i}^{k}$ are not elements of $\left\{\mathcal{P}_{i}: i \in I\right\}$, but satisfy for $i \geqq i_{0}$

$$
\left|P_{i, j} \triangle P_{i, j}^{k}\right| \leqq\left|\bigcup_{l \geqq k} P_{i_{0}, l}\right| \leqq \omega(k) \text { for all } j \geqq 0
$$


with $\omega(k) \rightarrow 0$ for $k \rightarrow \infty$, where we set $P_{i, 0}=\emptyset$. For all $k \in \mathbb{N}$ we observe that $\left\{\mathcal{P}_{i}^{k}: i \geqq i_{0}\right\}$ contains only a finite number of different elements and therefore contains a maximal element $\mathcal{P}^{k}=\left(P_{j}^{k}\right)_{j}$. Now we can choose $i_{0} \leqq i_{1} \leqq i_{2} \leqq \ldots$ such that $\mathcal{P}^{k}=\mathcal{P}_{i_{k}}^{k}$ for $k \in \mathbb{N}$. It now suffices to construct an upper bound $\mathcal{P}=$ $\left(P_{j}\right)_{j} \in \mathcal{Z}_{P}$ with $\mathcal{P} \geqq \mathcal{P}_{i_{k}}$ for all $k \in \mathbb{N}$. Indeed, we then obtain $\mathcal{P} \geqq \mathcal{P}_{i}$ for all $i_{0} \leqq i$ as follows:

For each $P_{i, j}$ and each $k \in \mathbb{N}$ we find $P_{j_{k}}$ with $\left|P_{i, j} \backslash P_{j_{k}}\right| \leqq 2 \omega(k)$. In fact, using repetitively (5.1) and (5.6) we get $j^{\prime}, j_{k}$ such that $\left|P_{i, j} \backslash P_{i, j}^{k}\right| \leqq \omega(k)$, $\left|P_{i, j}^{k} \backslash P_{i_{k}, j^{\prime}}^{k}\right|=0,\left|P_{i_{k}, j^{\prime}}^{k} \backslash P_{i_{k}, j^{\prime}}\right| \leqq \omega(k),\left|P_{i_{k}, j^{\prime}} \backslash P_{j_{k}}\right|=0$ and thus $\left|P_{i, j} \backslash P_{j_{k}}\right| \leqq$ $2 \omega(k)$. As $\omega(k) \rightarrow 0$ for $k \rightarrow \infty$ and $\mathcal{P}$ contains only a finite number of components with $\mathcal{L}^{2}$-measure larger than $\frac{1}{2}\left|P_{i, j}\right|$, we indeed find $P_{j_{*}}$ with $\left|P_{i, j} \backslash P_{j_{*}}\right|=0$, as desired.

Step II: Now consider the totally ordered sequence of partitions $\left(\mathcal{P}_{i_{k}}\right)_{k}$. For notational convenience we will denote the sequence by $\left(\mathcal{P}_{i}\right)_{i \in \mathbb{N}}$ in the following. By the compactness theorem for Caccioppoli partitions (see Theorem 3.6) we get an (ordered) Caccioppoli partition $\mathcal{P}=\left(P_{j}\right)_{j}$ such that $\left|P_{i, j} \triangle P_{j}\right| \rightarrow 0$ for $i \rightarrow \infty$ for all $j \in \mathbb{N}$. Thus, for all $j \in \mathbb{N}$ there exists $I_{j} \in \mathbb{N}$ such that $\left|P_{i_{1}, j} \backslash P_{i_{2}, j}\right| \leqq \frac{1}{2}\left|P_{i_{1}, j}\right|$ for all $I_{j} \leqq i_{1} \leqq i_{2}$. As $\left(\mathcal{P}_{i}\right)_{i \in \mathbb{N}}$ is totally ordered, (5.1) then gives $\left|P_{i_{1}, j} \backslash P_{i_{2}, j}\right|=0$ for all $I_{j} \leqq i_{1} \leqq i_{2}$ and this monotonicity yields $\left|P_{i_{1}, j} \backslash P_{j}\right|=0$ for $i_{1} \geqq I_{j}$. Eventually, fixing $P_{i, j}$ for $i, j \in \mathbb{N}$, by the above arguments there exists $j^{\prime} \in \mathbb{N}$ such that $\left|P_{i, j} \backslash P_{i^{\prime}, j^{\prime}}\right|=0$ for all $i^{\prime}$ large enough and thus $\left|P_{i, j} \backslash P_{j^{\prime}}\right|=0$.

This implies $\mathcal{P} \geqq \mathcal{P}_{i}$ for all $i \in \mathbb{N}$ and therefore it suffices to show that $\mathcal{P} \in \mathcal{Z}_{P}$. To this end, we will construct partitions $\mathcal{P}^{n}$, rigid motions $T_{n} \in \mathcal{R}\left(\mathcal{P}^{n}\right)$ and a limiting function $u$ by a diagonal sequence argument.

For all $i \in \mathbb{N}$, according to Definition 2.5(i), we find $\left(u_{i}^{k}, \mathcal{P}_{i}^{k}, T_{i}^{k}\right) \in \mathcal{D}$, an admissible limiting configurations $u_{i} \in \mathcal{Z}_{u}\left(\mathcal{P}_{i}\right)$ and $T \in \mathcal{Z}_{T}$ as in Lemma 5.1 such that (2.6)-(2.9) hold as $k \rightarrow \infty$. The strategy is to select $u_{i}$ in a suitable way such that we find a limiting configuration $u \in G S B(\Omega)$ with

$$
\begin{aligned}
& u_{i} \rightarrow u \text { almost everywhere in } \Omega, \\
& e\left(\nabla T^{T} \nabla u_{i}\right) \rightarrow e\left(\nabla T^{T} \nabla u\right) \quad \text { weakly in } L^{2}\left(\Omega, \mathbb{R}_{\mathrm{sym}}^{2 \times 2}\right), \\
& \liminf _{i \rightarrow \infty} \mathcal{H}^{1}\left(J_{u_{i}}\right) \geqq \mathcal{H}^{1}\left(J_{u}\right) .
\end{aligned}
$$

We defer the selection of the sequence $\left(u_{i}\right)_{i}$ to Step III below. Then we can choose a diagonal sequence $\left(\bar{u}_{n}\right):=\left(u_{n}^{k(n)}\right)_{n}$ converging to the triple $(u, \mathcal{P}, T)$ in the sense of (2.6)-(2.9). Indeed, $k(n)$ can be selected such that letting $\overline{\mathcal{P}}^{n}=\left(\bar{P}_{j}^{n}\right)_{j}=$ $\mathcal{P}_{n}^{k(n)}$ and $\bar{T}_{n}=T_{n}^{k(n)} \in \mathcal{R}\left(\overline{\mathcal{P}}^{n}\right)$, we find $\left|\bar{P}_{j}^{n} \triangle P_{j}\right| \rightarrow 0$ for all $j \in \mathbb{N}$ (even $\sum_{j}\left|\bar{P}_{j}^{n} \triangle P_{j}\right| \rightarrow 0$, cf. below Theorem 3.6) and $\bar{T}_{n} \rightarrow T, \nabla \bar{T}_{n} \rightarrow \nabla T$ in $L^{2}(\Omega)$. This gives (2.7). Moreover, as measure convergence is metrizable, this can be done in a way that $\bar{u}_{n} \rightarrow u$ in measure and $\bar{u}_{n}-\varepsilon_{n}^{-1 / 2}\left(y_{n}-\bar{T}_{n}\right) \rightarrow 0$ in measure. Then, possibly passing to a further subsequence, we can assume that the convergence also holds almost everywhere in $\Omega$ and thus (2.6)(i), (2.8)(i) are satisfied.

Likewise, (2.8)(ii) can be achieved by (5.7) and the fact the weak convergence is metrizable as by Remark 4.3 we get $\left\|e\left(\left(\nabla T_{i}^{k}\right)^{T} \nabla u_{i}^{k}\right)\right\|_{L^{2}(\Omega)} \leqq C$ for a con- 
stant independent of $k, i$. Moreover, (2.6)(ii) and (2.9)(i) directly follow from the corresponding estimates for the functions $u_{i}^{k}$. Finally, to see (2.9)(ii) it suffices to prove

$$
\liminf _{i \rightarrow \infty}\left(\mathcal{H}^{1}\left(J_{u_{i}} \backslash \partial P_{i}\right)+\mathcal{H}^{1}\left(\partial P_{i} \cap \Omega\right)\right) \geqq \mathcal{H}^{1}\left(J_{u} \backslash \partial P\right)+\mathcal{H}^{1}(\partial P \cap \Omega),
$$

where for shorthand $\partial P_{i}=\bigcup_{j} \partial^{*} P_{i, j}$ and $\partial P=\bigcup_{j} \partial^{*} P_{j}$. This can be derived arguing as in (4.20). We may consider an infinitesimal perturbation of the form $\tilde{u}_{i}=u_{i}+\sum_{j} b_{j} \chi_{P_{i, j}}, \tilde{u}=u+\sum_{j} b_{j} \chi_{P_{j}}$ with $b_{j}$ small such that $\mathcal{H}^{1}\left(\partial P_{i} \backslash J_{\tilde{u}_{i}}\right)=$ $\mathcal{H}^{1}\left(\partial P \backslash J_{\tilde{u}}\right)=0$ and the convergence in (5.7) still holds after replacing $u_{i}, u$ by $\tilde{u}_{i}, \tilde{u}$, respectively. Then the claim follows from (5.7). Consequently, $\mathcal{P} \in \mathcal{Z}_{P}$ due to Definition 2.5(i).

Step III: It remains to show (5.7). Clearly, we have $\left\|e\left(\nabla T^{T} \nabla u_{i}\right)\right\|_{L^{2}(\Omega)}^{2} \leqq$ $C$ and $\mathcal{H}^{1}\left(J_{u_{i}}\right) \leqq C$ for a constant independent of $i \in \mathbb{N}$. This follows by a lower semicontinuity argument using (2.9)(ii) and $\left\|e\left(\left(\nabla T_{i}^{k}\right)^{T} \nabla u_{i}^{k}\right)\right\|_{L^{2}(\Omega)} \leqq C$ by Remark 4.3. Thus, in order to apply Theorem 3.2, we have to select $u_{i} \in \mathcal{Z}_{u}\left(\mathcal{P}_{i}\right)$ suitably and to find an increasing continuous function $\psi:[0, \infty) \rightarrow[0, \infty)$ with $\lim _{t \rightarrow \infty} \psi(t)=+\infty$ such that $\int_{\Omega} \psi\left(\left|u_{i}\right|\right) \leqq C$ independently of $i \in \mathbb{N}$.

We proceed similarly as in the proof of Theorem 2.2 and define $u_{i}$ iteratively. Choose $u_{1} \in \mathcal{Z}_{u}\left(\mathcal{P}_{1}\right)$ arbitrarily. Given $u_{i}$ we define $u_{i+1}$ as follows. Consider some $P_{i+1, j}$ and recalling (5.1) choose $l_{1, j}<l_{2, j}<\ldots$ such that $P_{i+1, j}=$ $\bigcup_{k=1}^{\infty} P_{i, l_{k, j}}$ up to an $\mathcal{L}^{2}$ - negligible set (observe that the union may also be finite). Choose some $\tilde{u}_{i+1} \in \mathcal{Z}_{u}\left(\mathcal{P}_{i+1}\right)$. By Lemma 5.4 for $\mathcal{P}=\mathcal{P}_{i}, \hat{\mathcal{P}}=\mathcal{P}_{i+1}$ we get $\left(u_{i}-\tilde{u}_{i+1}\right) \chi_{P_{i+1, j}}=\sum_{k=1}^{\infty} \nabla T\left(A_{l_{k, j}}+b_{l_{k, j}}\right) \chi_{P_{i, l_{k, j}}}$ for $A_{l_{k, j}} \in \mathbb{R}_{\mathrm{skew}}^{2 \times 2}, b_{l_{k, j}} \in \mathbb{R}^{2}$. Now define

$$
u_{i+1}(x)=\tilde{u}_{i+1}(x)+\nabla T(x)\left(A_{l_{1, j}} x+b_{l_{1, j}}\right)
$$

for $x \in P_{i+1, j}$ and observe that $u_{i}=u_{i+1}$ on $P_{i, l_{1, j}}$. Proceeding in this way on all $P_{i+1, j}$ we find some $\tilde{A}^{i+1} \in \mathcal{A}\left(\mathcal{P}_{i+1}\right)$ such that $u_{i+1}:=\tilde{u}_{i+1}+\nabla T \tilde{A}^{i+1} \in$ $\mathcal{Z}_{u}\left(\mathcal{P}_{i+1}\right)$ applying Lemma 5.4 for $\mathcal{P}=\hat{\mathcal{P}}=\mathcal{P}_{i+1}$. Moreover, there is a corresponding $A^{i} \in \mathcal{A}\left(\mathcal{P}_{i}\right)$ such that $u_{i+1}=u_{i}+\nabla T A^{i}$ with $A^{i}=0$ on $\bigcup_{j} P_{i, l_{1, j}}$.

We now show that $\sum_{i \in \mathbb{N}}\left|A^{i}(x)\right|<+\infty$ for almost every $x \in \Omega$. To see this, we recall that $\left|P_{i, j} \triangle P_{j}\right| \rightarrow 0$ for all $j \in \mathbb{N}$. Consequently, as due to the total order of the partitions the sets $P_{i, j}$ are increasing for fixed $j \in \mathbb{N}$, the construction of the functions $\left(u_{i}\right)_{i}$ implies $A^{i}=0$ on $P_{i, j}$ for $i$ so large that $\left|P_{i, j}\right|>\frac{1}{2}\left|P_{j}\right|$. Thus, for almost every $x \in P_{j}$ the sum $\sum_{i \geqq 1}\left|A^{i}(x)\right|$ is a finite sum and therefore finite. As $j \in \mathbb{N}$ was arbitrary, we obtain $\sum_{i \in \mathbb{N}}\left|A^{i}\right|<+\infty$ almost everywhere.

Therefore, the function $v:=\left|u_{1}\right|+\sum_{l \in \mathbb{N}}\left|A^{l}\right|$ is finite almost everywhere in $\Omega$ and we apply Lemma 4.2 on the sequence $v_{k}=v \chi_{\{v \leq k\}}$ to find an increasing continuous function $\psi:[0, \infty) \rightarrow[0, \infty)$ with $\lim _{t \rightarrow \infty} \psi(t)=\infty$ such that by Fatou's lemma $\|\psi(v)\|_{L^{1}(\Omega)} \leqq \liminf _{k \rightarrow \infty}\left\|\psi\left(v_{k}\right)\right\|_{L^{1}(\Omega)} \leqq C<\infty$. Using the definition $u_{i+1}=u_{i}+\nabla T A^{i}$ and the monotonicity of $\psi$ we find $\left\|\psi\left(\left|u_{i}\right|\right)\right\|_{L^{1}(\Omega)} \leqq$ $\left\|\psi\left(\left|u_{1}\right|+\sum_{l \in \mathbb{N}}\left|A^{l}\right|\right)\right\|_{L^{1}(\Omega)} \leqq C<\infty$ for all $i \in \mathbb{N}$, as desired. 
After these preparatory lemmas we are in a position to prove Theorem 2.6.

Proof of Theorem 2.6. First, (i) follows from Lemma 5.1. The uniqueness of the coarsest partition is a consequence of Lemma 5.3 and Lemma 5.2. We obtain existence by Zorn's lemma: As $\left(\mathcal{Z}_{P}, \geqq\right)$ is a partial order and every chain has an upper bound by Lemma 5.5, there exists a maximal element $\overline{\mathcal{P}} \in \mathcal{Z}_{P}$. Lemma 5.2 shows that $\overline{\mathcal{P}}$ is a coarsest partition which gives (ii). Finally, assertion (iii), namely $\mathcal{Z}_{u}(\overline{\mathcal{P}})=v+\nabla T \mathcal{A}(\overline{\mathcal{P}})$ for some $v \in \mathcal{Z}_{u}(\overline{\mathcal{P}})$, follows from Lemma 5.4 for the choice $\mathcal{P}=\hat{\mathcal{P}}=\overline{\mathcal{P}}$.

\section{The Effective Linearized Griffith Model}

In this final section we identify the effective linearized Griffith functional via $\Gamma$-convergence and derive a cleavage law for the limiting model.

\subsection{Derivation of Linearized Models via $\Gamma$-Convergence}

We now give the proof of Theorem 2.7.

Proof of Theorem 2.7. (i) Thanks to the preparations in the last section the lower bound is almost immediate. Let $(u, \mathcal{P}, T) \in \mathcal{D}_{\infty}$ be given as well as a sequence $\left(y_{k}\right)_{k} \subset S B V_{M}(\Omega)$ with $y_{k} \rightarrow(u, \mathcal{P}, T)$, that is by Definition 2.3 the are triples $\left(u_{k}, \mathcal{P}_{k}, T_{k}\right) \in \mathcal{D}$ such that (2.6)-(2.9) hold. Due to (2.9)(ii) it suffices to show

$$
\liminf _{k \rightarrow \infty} \frac{1}{\varepsilon_{k}} \int_{\Omega} W\left(\nabla y_{k}\right) \geqq \int_{\Omega} \frac{1}{2} Q\left(e\left(\nabla T^{T} \nabla u\right)\right) .
$$

We proceed as in (4.9). Recall that $W(G)=\frac{1}{2} Q(e(G-\mathbf{I d}))+\omega_{W}(G-\mathbf{I d})$ with $\sup \left\{|F|^{-3} \omega_{W}(F):|F| \leqq 1\right\} \leqq C$ by the assumptions on $W$, where $Q=$ $D^{2} W($ Id). We compute by (2.9)(i)

$$
\begin{aligned}
\frac{1}{\varepsilon_{k}} \int_{\Omega} W\left(\nabla y_{k}\right) & \geqq \frac{1}{\varepsilon_{k}} \int_{\Omega} W\left(\mathbf{I d}+\sqrt{\varepsilon_{k}} \nabla T_{k}^{T} \nabla u_{k}\right)+o(1) \\
& =\int_{\Omega} \frac{1}{2}\left(Q\left(e\left(\nabla T_{k}^{T} \nabla u_{k}\right)\right)+\frac{1}{\varepsilon_{k}} \omega_{W}\left(\sqrt{\varepsilon_{k}} \nabla T_{k}^{T} \nabla u_{k}\right)\right)+o(1)
\end{aligned}
$$

as $k \rightarrow \infty$. The second term converges to 0 arguing as in (4.10) and using $\left\|\nabla u_{k}\right\|_{\infty} \leqq c \varepsilon_{k}^{-1 / 8}$ (see (2.6)(ii)). As $e\left(\nabla T_{k}^{T} \nabla u_{k}\right) \rightarrow e\left(\nabla T^{T} \nabla u\right)$ weakly in $L^{2}\left(\Omega, \mathbb{R}_{\text {sym }}^{2 \times 2}\right)$ by (2.8)(ii) and $Q$ is convex, we conclude

$$
\liminf _{k \rightarrow \infty} \frac{1}{\varepsilon_{k}} \int_{\Omega} W\left(\nabla y_{k}\right) \geqq \int_{\Omega} \frac{1}{2} Q\left(e\left(\nabla T^{T} \nabla u\right)\right) .
$$

(ii) By a general density result in the theory of $\Gamma$-convergence together with Theorem 3.4 and the fact that the limiting functional $E(u, \mathcal{P}, T)$ is continuous in $u$ with respect to the convergence given in Theorem 3.4 , it suffices to provide recovery sequences for functions $u$ with $u \in W^{1, \infty}\left(\Omega \backslash \overline{J_{u}}\right)$, where $J_{u}$ is contained 
in the union of a finite number of closed connected pieces of $C^{1}$ - curves. Moreover, as in the proof of Theorem 2.2 (see paragraph before (4.20)) we may assume that $\mathcal{H}^{1}\left(\left(\bigcup_{j} \partial^{*} P_{j} \cap \Omega\right) \backslash J_{u}\right)=0$ up to an infinitesimal small perturbation of $u$ (a similar argument was used below (5.8)).

Let $(u, \mathcal{P}, T) \in \mathcal{D}_{\infty}$ and $\varepsilon_{k} \rightarrow 0$ be given. Define $y_{k}(x)=T(x)+\sqrt{\varepsilon_{k}} u(x)$ for all $x \in \Omega$. It is not hard to see that $\left(y_{k}\right)_{k} \subset S B V_{M}(\Omega)$ for $\varepsilon_{k}$ small enough (and $M$ not too small). Moreover, define $\mathcal{P}_{k}=\mathcal{P}, T_{k}=T$ and $u_{k}=\varepsilon_{k}^{-1 / 2}\left(y_{k}-T_{k}\right) \equiv u$ for all $k \in \mathbb{N}$. Then (2.6)(i) and (2.7)-(2.9) hold trivially. To see (2.6)(ii), it suffices to note that $\left\|\nabla u_{k}\right\|_{\infty}=\|\nabla u\|_{\infty} \leqq C \leqq C \varepsilon_{k}^{-1 / 8}$. Consequently, $y_{k} \rightarrow(u, \mathcal{P}, T)$ in the sense of Definition 2.3.

We finally confirm $\lim _{k \rightarrow \infty} E_{\varepsilon_{k}}\left(y_{k}\right)=E(u, \mathcal{P}, T)$. As clearly $\lim _{k \rightarrow \infty} \mathcal{H}^{1}\left(J_{y_{k}}\right)=\mathcal{H}^{1}\left(\bigcup_{j} \partial^{*} P_{j} \cap \Omega\right)+\mathcal{H}^{1}\left(J_{u} \backslash \bigcup_{j} \partial^{*} P_{j}\right)$, it suffices to show $\lim _{k \rightarrow \infty} \frac{1}{\varepsilon_{k}} \int_{\Omega} W\left(\nabla y_{k}\right)=\int_{\Omega} \frac{1}{2} Q\left(e\left(\nabla T^{T} \nabla u\right)\right)$. Using again that $W(G)=$ $\frac{1}{2} Q(e(G-\mathbf{I d}))+\omega_{W}(G-\mathbf{I d})$ and the frame indifference of $W$ we compute

$$
\begin{aligned}
\frac{1}{\varepsilon_{k}} \int_{\Omega} W\left(\nabla y_{k}\right) & =\frac{1}{\varepsilon_{k}} \int_{\Omega} W\left(\mathbf{I d}+\sqrt{\varepsilon_{k}} \nabla T_{k}^{T} \nabla u_{k}\right) \\
& =\int_{\Omega}\left(\frac{1}{2} Q\left(e\left(\nabla T_{k}^{T} \nabla u_{k}\right)\right)+\frac{1}{\varepsilon_{k}} \omega_{W}\left(\sqrt{\varepsilon_{k}} \nabla T_{k}^{T} \nabla u_{k}\right)\right) \\
& =\int_{\Omega} \frac{1}{2} Q\left(e\left(\nabla T^{T} \nabla u\right)\right)+O\left(\sqrt{\varepsilon_{k}}\right) \rightarrow \int_{\Omega} \frac{1}{2} Q\left(e\left(\nabla T^{T} \nabla u\right)\right) .
\end{aligned}
$$

This concludes the proof.

The proof of Corollary 2.9 is now straightforward.

Proof of Corollary 2.9. To see the liminf-inequality, assume $y_{\varepsilon_{k}} \rightarrow y$ in $L^{1}(\Omega)$ for $k \rightarrow \infty$ and without restriction that $E_{\varepsilon_{k}}\left(y_{\varepsilon_{k}}\right) \leqq C$. By Theorem 2.2 we find a limiting triple $(u, \mathcal{P}, T) \in \mathcal{D}_{\infty}$ such that $y_{\varepsilon_{k}} \rightarrow(u, \mathcal{P}, T)$ in the sense of Definition 2.3. By (2.6)(i), (2.7)(ii) we obtain $y=T$. As $T \in \mathcal{R}(\mathcal{P})$, we get $\mathcal{H}^{1}\left(J_{T}\right) \leqq \mathcal{H}^{1}\left(\bigcup_{j} \partial^{*} P_{j} \cap \Omega\right)$, where $\mathcal{P}=\left(P_{j}\right)_{j}$. Thus, Theorem 2.7(i) yields $\liminf _{k \rightarrow \infty} E_{\varepsilon_{k}}\left(y_{k}\right) \geqq E(u, \mathcal{P}, T) \geqq \mathcal{H}^{1}\left(J_{T}\right)=E_{\text {seg }}(y)$. A recovery sequence is obviously given by $y_{k}=y$ for all $k \in \mathbb{N}$.

We close this section with the proof of Lemma 2.10 and Theorem 2.11.

Proof of Lemma 2.10. Consider triples $\left(u_{k}, \mathcal{P}_{k}, T_{k}\right)$ and $\left(g_{k}, \mathcal{P}_{k}^{g}, T_{k}^{g}\right)$ such that the triples converge to $(u, \mathcal{P}, T)$ and $\left(g, \mathcal{P}_{g}, T_{g}\right)$, respectively, in the sense of (2.6)(2.9). Since $\varepsilon_{k}^{-1 / 2}\left(y_{k}-f_{k}\right)$ is bounded in $L^{2}(\Omega)$ by (2.12), $\varepsilon_{k}^{-1 / 2}\left(T_{k}-T_{k}^{g}\right)+$ $\left(u_{k}-g_{k}\right)-\varepsilon_{k}^{-1 / 2}\left(y_{k}-f_{k}\right)$ converges almost everywhere by (2.6)(i), and $u_{k}-g_{k}$ converges almost everywhere by (2.8)(i), we get $\varepsilon_{k}^{-1 / 2}\left(T_{k}-T_{k}^{g}\right)$ converges (up to a subsequence) in measure on $\Omega$. This implies $T=T_{g}$ by (2.7)(ii).

Moreover, suppose that $\mathcal{P}_{g} \geqq \mathcal{P}$ was wrong (recall (5.1)). We may then assume after reordering that $P_{1}^{g} \cap \bar{P}_{1}$ and $P_{2}^{g} \cap P_{1}$ have positive $\mathcal{L}^{2}$-measure. Since $\varepsilon_{k}^{-1 / 2}\left(T_{k}-T_{k}^{g}\right)$ converges in measure on $P_{1}$, we can argue exactly as in the proof 
of Lemma 5.2(2) to see that the partition $\mathcal{P}_{g}$ is not coarsest, which contradicts the assumption.

Proof of Theorem 2.11. First consider the lower bound. Let $(u, \mathcal{P}, T) \in \mathcal{D}_{\infty}$ be given as well as a sequence $\left(y_{k}\right)_{k} \subset S B V_{M}(\Omega)$ with $y_{k} \rightarrow(u, \mathcal{P}, T)$. If $(u, \mathcal{P}, T) \notin$

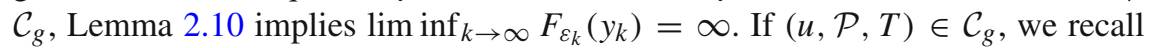
Theorem 2.7(i) and see that it suffices to show

$$
\liminf _{k \rightarrow \infty} \varepsilon_{k}^{-1}\left\|y_{k}-f_{k}\right\|_{L^{2}(\Omega)}^{2} \geqq \min _{v \in u+\nabla T \mathcal{A}(\mathcal{P})}\|v-g\|_{L^{2}(\Omega)}^{2} .
$$

Consider the triples $\left(u_{k}, \mathcal{P}_{k}, T_{k}\right)$ and $\left(g_{k}, \mathcal{P}_{k}^{g}, T_{k}^{g}\right)$ as in the previous proof. Recall $\varepsilon_{k}^{-1 / 2}\left(y_{k}-f_{k}\right)-\varepsilon_{k}^{-1 / 2}\left(T_{k}-T_{k}^{g}\right)-\left(u_{k}-g_{k}\right) \rightarrow 0$ almost everywhere by (2.6)(i) and $u_{k}-g_{k} \rightarrow u-g$ almost everywhere by (2.8)(i). Now it is enough to show that for each $P_{j}$ there are $A_{j} \in \mathbb{R}_{\text {skew }}^{2 \times 2}$ and $d_{j} \in \mathbb{R}^{2}$ such that $\varepsilon_{k}^{-1 / 2}\left(T_{k}-T_{k}^{g}\right) \rightarrow$ $\nabla T\left(A_{j} \cdot+d_{j}\right)$ almost everywhere on $P_{j}$. Then (6.2) follows from Fatou's lemma. We recall from the proof of Lemma 2.10 that $\varepsilon_{k}^{-1 / 2}\left(T_{k}-T_{k}^{g}\right)$ converges in measure on $P_{j}$ and that $P_{j} \subset P_{i}^{g}$ for some $P_{i}^{g}$. In particular, this implies $\left|R_{j}^{k}-R_{i}^{g, k}\right|+$ $\left|b_{j}^{k}-b_{i}^{g, k}\right| \leqq C \sqrt{\varepsilon_{k}}$, where $R_{i}^{g, k} \cdot+b_{i}^{g, k}$ denotes the rigid motion associated to $T_{k}^{g}$. Repeating the argument in (5.2) we obtain the desired convergence.

For the construction of recovery sequences we mainly follow Theorem 2.7(ii) and only indicate the necessary adaptions. Let $(u, \mathcal{P}, T) \in \mathcal{C}_{g}$ be given with $u$ having the specific regularity assumed in Theorem 2.7(ii), particularly $\nabla u \in L^{\infty}(\Omega)$. Let $A \in \mathcal{A}(\mathcal{P})$ such that $v:=u+\nabla T A$ realizes the minimum in (6.2).

As $u, g \in L^{2}(\Omega)$ and thus $\nabla T A \in L^{2}(\Omega)$, we can choose a sequence $\left(A^{k}\right)_{k} \subset$ $\mathcal{A}\left(\mathcal{P}_{k}^{g}\right)$ such that $\nabla T_{k}^{g} A^{k} \rightarrow \nabla T_{g} A=\nabla T A$ in $L^{2}(\Omega)$ and $\sqrt{\varepsilon_{k}}\left|\nabla A^{k}\right|^{2} \rightarrow 0$ in $L^{2}(\Omega)$. Select $T_{k}=\sum_{j}\left(R_{j}^{k} \cdot+b_{j}^{k}\right) \chi_{P_{j}^{g, k}} \in \mathcal{R}\left(\mathcal{P}_{k}^{g}\right)$ such that (cf. before (5.5) for a similar construction)

$$
\operatorname{dist}\left(\nabla\left(T_{k}^{g}+\sqrt{\varepsilon_{k}} \nabla T_{k}^{g} A^{k}\right), S O(2)\right)=\left|\nabla\left(T_{k}^{g}+\sqrt{\varepsilon_{k}} \nabla T_{k}^{g} A^{k}\right)-\nabla T_{k}\right| \text { on } \Omega
$$

and on each component the translations of $T_{k}$ and $T_{k}^{g}+\sqrt{\varepsilon_{k}} \nabla T_{k}^{g} A^{k}$ coincide, that is

$$
b_{j}^{k}=T_{k}(x)-\nabla T_{k}(x) x=\left(T_{k}^{g}+\sqrt{\varepsilon_{k}} \nabla T_{k}^{g} A^{k}\right)(x)-\nabla\left(T_{k}^{g}+\sqrt{\varepsilon_{k}} \nabla T_{k}^{g} A^{k}\right)(x) x
$$

for all $x \in P_{j}^{g, k}$ and all $j \in \mathbb{N}$. Note that using (3.12) a short calculation implies

$$
\left|T_{k}^{g}+\sqrt{\varepsilon_{k}} \nabla T_{k}^{g} A^{k}-T_{k}\right|+\left|\nabla T_{k}^{g}+\sqrt{\varepsilon_{k}} \nabla T_{k}^{g} \nabla A^{k}-\nabla T_{k}\right| \leqq C \varepsilon_{k}\left|\nabla A^{k}\right|^{2}
$$

pointwise almost everywhere in $\Omega$. We define $y_{k}=T_{k}+\sqrt{\varepsilon_{k}} u, u_{k}=u$ and $\mathcal{P}_{k}=\mathcal{P}$ for all $k \in \mathbb{N}$. We note that $T_{k} \in \mathcal{R}\left(\mathcal{P}_{k}\right)$ since $T_{k} \in \mathcal{R}\left(\mathcal{P}_{k}^{g}\right), \mathcal{P}_{k} \leqq \mathcal{P}_{g}$ by $(u, \mathcal{P}, T) \in$ $\mathcal{C}_{g}$ and $\mathcal{P}_{k}^{g}=\mathcal{P}^{g}$ by assumption. Moreover, $\nabla T_{k}^{T} \nabla y_{k}=\mathbf{I d}+\sqrt{\varepsilon_{k}} \nabla T_{k}^{T} \nabla u$ and by $(6.3)$

$$
\nabla T_{k}^{T} \nabla u=\left(\nabla T_{k}^{g}\right)^{T} \nabla u+O\left(\|\nabla u\|_{\infty} \varepsilon_{k}\left|\nabla A^{k}\right|^{2}\right)+O\left(\|\nabla u\|_{\infty} \sqrt{\varepsilon_{k}}\left|\nabla A^{k}\right|\right) .
$$


Using $\sqrt{\varepsilon_{k}}\left|\nabla A^{k}\right|^{2} \rightarrow 0$ in $L^{2}(\Omega)$ and $T_{k}^{g} \rightarrow T, \nabla T_{k}^{g} \rightarrow \nabla T$ in $L^{2}(\Omega)$, one can check that $\left(y_{k}\right)_{k} \subset S B V_{M}(\Omega)$ and that (2.6)-(2.9) hold, that is $y_{k} \rightarrow(u, \mathcal{P}, T)$ (see also proof of Theorem 2.7). Likewise, (6.4) together with the calculation in (6.1) shows that $\lim _{k \rightarrow \infty} E_{\varepsilon_{k}}\left(y_{k}\right)=E(u, \mathcal{P}, T)$. Finally, recalling $\varepsilon_{k}^{-1 / 2}\left(f_{k}-T_{k}^{g}\right) \rightarrow g$ in $L^{2}(\Omega)$ (see before Lemma 2.10), $\nabla T_{k}^{g} A^{k} \rightarrow \nabla T A$ in $L^{2}(\Omega)$ and (6.3), we conclude

$$
\begin{aligned}
\varepsilon_{k}^{-1 / 2}\left\|y_{k}-f_{k}\right\|_{L^{2}(\Omega)}= & \left\|\varepsilon_{k}^{-1 / 2}\left(T_{k}-T_{k}^{g}\right)+u-\varepsilon_{k}^{-1 / 2}\left(f_{k}-T_{k}^{g}\right)\right\|_{L^{2}(\Omega)} \\
= & \left\|\nabla T_{k}^{g} A^{k}+u-\varepsilon_{k}^{-1 / 2}\left(f_{k}-T_{k}^{g}\right)\right\|_{L^{2}(\Omega)} \\
& +O\left(\left\|\varepsilon_{k}^{1 / 2}\left|\nabla A^{k}\right|^{2}\right\|_{L^{2}(\Omega)}\right) \rightarrow \\
& \|\nabla T A+u-g\|_{L^{2}(\Omega)}=\|v-g\|_{L^{2}(\Omega)} .
\end{aligned}
$$

Finally, the convergence result for minimum problems and minimizers follows from a general result in the theory of $\Gamma$-convergence (see [17, Chapter 7]).

\subsection{An Application to Cleavage Laws}

We are finally in a position to prove the cleavage law in Theorem 2.12. Analogous results for the case of expansive boundary values have been obtained in [37] and [31]. We thus do not repeat all the steps of these proofs but rather concentrate on the additional arguments necessary in our general setting (see (2.11)) in which we particularly can extend the aforementioned results to the case of compression.

Proof of Theorem 2.12. Let $\left(y_{\varepsilon_{k}}\right)_{k}$ be a sequence of almost minimizers. Passing to a suitable subsequence, by Theorem 2.2 we obtain a triple $\left(u_{k}, \mathcal{P}_{k}, T_{k}\right) \in \mathcal{D}$ and a limiting triple $(u, \mathcal{P}, T) \in \mathcal{D}_{\infty}$ such that (2.6)-(2.9) hold and

$$
E(u, \mathcal{P}, T) \leqq \liminf _{\varepsilon \rightarrow 0} \inf \left\{E_{\varepsilon}(y): y \in \mathcal{A}\left(a_{\varepsilon}\right)\right\}
$$

by Theorem 2.7(i). Write $T_{k}=\sum_{j}\left(R_{j}^{k} \cdot+b_{j}^{k}\right) \chi_{P_{j}^{k}}$ and $\mathcal{P}=\left(P_{j}\right)_{j}$. Due to the boundary conditions and (2.6)(i),(2.8)(i), on each component $P_{j} \in \mathcal{P}$ we find $A_{j} \in \mathbb{R}_{\text {skew }}^{2 \times 2}$ and $b_{j} \in \mathbb{R}^{2}$ such that

$$
\begin{aligned}
u_{1}(x) & =\lim _{k \rightarrow \infty} \varepsilon_{k}^{-1 / 2}\left(\mathbf{e}_{1} \cdot\left(\mathbf{I d}-R_{j}^{k}\right) x-\mathbf{e}_{1} \cdot b_{j}^{k}+a_{\varepsilon_{k}} x_{1}\right) \\
& =\mathbf{e}_{1} \cdot A_{j} x+\mathbf{e}_{1} \cdot b_{j}+a x_{1}
\end{aligned}
$$

for almost every $x \in \Omega^{\prime}$ with $x_{1}<0$ or $x_{1}>l$ and $x \in P_{j}$. In particular, this implies

$$
u_{1}\left(x_{1}, x_{2}\right)-u_{1}\left(\hat{x}_{1}, x_{2}\right)=\left|x_{1}-\hat{x}_{1}\right| a
$$

for almost every $x \in \Omega^{\prime}$ with $\hat{x}_{1}<0, x_{1}>l$ and $\left(x_{1}, x_{2}\right),\left(\hat{x}_{1}, x_{2}\right) \in P_{j}$.

We first derive the limiting minimal energy and postpone the characterization of the sequence of almost minimizers to the end of the proof. The argument in (6.5) shows that $\nabla T=\mathbf{I d}$ on $P_{j}$ if $\mid P_{j} \cap\left\{x: x_{1}<0\right.$ or $\left.x_{1}>l\right\} \mid>0$. It is not restrictive to assume $\nabla T^{T} \nabla u=\nabla u$ almost everywhere. Indeed, we may replace $u$ by 0 in 
a component $P_{j}$ which does not intersect the boundaries without increasing the energy. By (2.11), a slicing argument in GSBD (see Theorem 3.1) and the fact that $\inf \left\{Q(F): \mathbf{e}_{1}^{T} F \mathbf{e}_{1}=a\right\}=\alpha a^{2}$ (see Section 2.6) we obtain

$$
\begin{aligned}
E(u, \mathcal{P}, T) & \geqq \int_{\Omega} \frac{1}{2} Q(e(\nabla u))+\int_{J_{u}}\left|v_{u} \cdot \mathbf{e}_{1}\right| \mathrm{d} \mathcal{H}^{1}+\mathcal{E}(u) \\
& \geqq \int_{0}^{1}\left(\int_{0}^{l} \frac{\alpha}{2}\left(\mathbf{e}_{1}^{T} \nabla u(x) \mathbf{e}_{1}\right)^{2} \mathrm{~d} x_{1}+S^{x_{2}}(u)\right) \mathrm{d} x_{2}+\mathcal{E}(u),
\end{aligned}
$$

where $S^{x_{2}}$ denotes the number of jumps of $u_{1}$ on a slice $(-\eta, l+\eta) \times\left\{x_{2}\right\}$ and $\mathcal{E}(u)=\int_{J_{u}}\left(1-\left|v_{u} \cdot \mathbf{e}_{1}\right|\right) \mathrm{d} \mathcal{H}^{1}$. If $S^{x_{2}} \geqq 1$, the inner integral is bounded from below by 1 . By the structure theorem for Caccioppoli partitions (see Theorem 3.5) we find that $((-\eta, 0) \cup(l, l+\eta)) \times\left\{x_{2}\right\} \subset P_{j}$ for some $j \in \mathbb{N}$ for $\mathcal{H}^{1}$-almost every $x_{2}$ with $S^{x_{2}}=0$. Consequently, if $\# S^{x_{2}}=0$, by applying Jensen's inequality we derive that the term is bounded from below by $\frac{1}{2} \alpha l a^{2}$ due to the boundary conditions (6.6). This implies $E(u) \geqq \min \left\{\frac{1}{2} \alpha l a^{2}, 1\right\}$.

Otherwise, it is not hard to see that the configurations $y_{\varepsilon_{k}}^{\mathrm{el}}(x)=x+F^{a_{\varepsilon_{k}}} x$ for $x \in \Omega^{\prime}$ satisfy $E_{\varepsilon_{k}}\left(y_{\varepsilon_{k}}^{\mathrm{el}}\right) \rightarrow \frac{1}{2} \alpha l a^{2}$ for $k \rightarrow \infty$. Likewise, we get $E_{\varepsilon_{k}}\left(y_{\varepsilon_{k}}^{\mathrm{cr}}\right)=1$ for all $k \in \mathbb{N}$, where $y_{\varepsilon_{k}}^{\mathrm{cr}}(x)=x \chi_{x_{1}<\frac{1}{2}}+\left(x+\left(l a_{\varepsilon_{k}}, 0\right)\right) \chi_{x_{1}>\frac{1}{2}}$ for $x \in \Omega$ and $y_{\varepsilon_{k}}^{\mathrm{cr}}=\left(x_{1}\left(1+a_{\varepsilon_{k}}\right), x_{2}\right)$ for $x \in \Omega^{\prime} \backslash \Omega$. This completes (2.13).

It remains to characterize the sequences of almost minimizers. Let $\left(y_{\varepsilon_{k}}\right)_{k}$ be a sequence of almost minimizers and $(u, \mathcal{P}, T) \in \mathcal{D}_{\infty}$ a limiting triple as considered before (6.5). Again we may suppose $\nabla T^{T} \nabla u=\nabla u$ almost everywhere. We let first $|a|<a_{\text {crit }}$ and follow the arguments in the proof of [31, Theorem 2.4]. Since $E(u, \mathcal{P}, T)=\frac{1}{2} \alpha l a^{2}$, we infer from (6.7) that $u$ has no jump on almost every slice $(-\eta, l+\eta) \times\left\{x_{2}\right\}$ and satisfies $\mathbf{e}_{1}^{T} \nabla u \mathbf{e}_{1}=a$ almost everywhere by the imposed boundary values and the strict convexity of the mapping $t \mapsto t^{2}$ on $\mathbb{R}$. Thus, if $J_{u} \neq \emptyset$, a crack normal must satisfy $v_{u}= \pm \mathbf{e}_{2} \mathcal{H}^{1}$-almost everywhere. Additionally, taking $\mathcal{E}(u)$ into account, we find $J_{u}=\emptyset$, up to an $\mathcal{H}^{1}$ negligible set, that is, $u \in H^{1}\left(\Omega^{\prime}\right)$. By the strict convexity of $Q$ on symmetric matrices and the boundary values (6.5) we see that the derivative has the form

$$
\nabla u(x)=F^{a}+A \text { for almost every } x \in \Omega
$$

for a suitable $A \in \mathbb{R}_{\text {skew }}^{2 \times 2}$. Since $\Omega$ is connected, we conclude

$$
u(x)=F^{a} x+A x+b
$$

for $x \in \Omega$ and some $b \in \mathbb{R}^{2}$. In particular, this implies $\mathcal{P}$ consists only of $P_{1}=\Omega^{\prime}$ and thus by (6.5) we get $A=\lim _{k \rightarrow \infty} \varepsilon_{k}^{-1 / 2}\left(\mathbf{I d}-R_{1}^{k}\right)$ and $\mathbf{e}_{1} \cdot b=$ $-\lim _{k \rightarrow \infty} \varepsilon_{k}^{-1 / 2} \mathbf{e}_{1} \cdot b_{1}^{k}$. Let $s=\lim _{k \rightarrow \infty} \mathbf{e}_{2} \cdot\left(\varepsilon_{k}^{-1 / 2} b_{1}^{k}+b\right)$, which exists by (2.6)(i), (2.8)(i). We now conclude by (2.6)(i), (2.8)(i) for almost every $x \in \Omega$

$$
\begin{aligned}
\bar{u}(x):=\lim _{k \rightarrow \infty} \varepsilon_{k}^{-1 / 2}\left(y_{\varepsilon_{k}}(x)-x\right) & =u(x)+\lim _{k \rightarrow \infty} \varepsilon_{k}^{-1 / 2}\left(\left(R_{1}^{k}-\mathbf{I d}\right) x+b_{1}^{k}\right) \\
& =u(x)-A x-b+(0, s)=(0, s)+F^{a} x,
\end{aligned}
$$


that is $\bar{u}$ fulfills Theorem 2.12(i). If $|a|>a_{\text {crit }}$, we again consider the lower bound (6.7) and now obtain that on almost every slice $(0, l) \times\left\{x_{2}\right\}$ a minimizer $u$ has precisely one jump and that $\mathbf{e}_{1}^{T} \nabla u \mathbf{e}_{1}=0$ almost everywhere. By the strict convexity of $Q$ on symmetric matrices we then derive that $\nabla u$ is antisymmetric almost everywhere. As a consequence, the linearized piecewise rigidity estimate for $S B D$ functions (see [15, Theorem A.1] or the remark below Theorem 3.9) yields that there is a Caccioppoli partition $\left(E_{i}\right)_{i}$ of $\Omega$ such that

$$
u(x)=\sum_{i}\left(A_{i} x+b_{i}\right) \chi_{E_{i}}(x) \text { and } J_{u}=\bigcup_{i} \partial^{*} E_{i} \cap \Omega,
$$

where $A_{i} \in \mathbb{R}_{\text {skew }}^{2 \times 2}$ and $b_{i} \in \mathbb{R}^{2}$. (Note that indeed the linearized rigidity estimate can also be applied in the $G S B D$-setting as it relies on a slicing argument and an approximation which is also available in the generalized framework, see [34, Section 3.3]. The only difference is that the approximation does not converge in $L^{1}$ but only pointwise almost everywhere, which does not affect the argument.)

As $\mathcal{E}(u)=0$, we also note that $v_{u}= \pm \mathbf{e}_{1}$ almost everywhere on $J_{u}$. Following the arguments in [37], in particular using regularity results for boundary curves of sets of finite perimeter and exhausting the sets $\partial^{*} E_{i}$ with Jordan curves, we find that

$$
J_{u}=\bigcup_{i} \partial^{*} E_{i} \cap \Omega \subset(p, 0)+\mathbb{R} \mathbf{e}_{1}
$$

for some $p \in(0, l)$. We thus obtain that $\left(E_{i}\right)_{i}$ consists of only two sets and $u$ has the form

$$
u(x)= \begin{cases}A_{1} x+b_{1} & \text { for } x_{1}<p, \\ A_{2} x+b_{2} & \text { for } x_{1}>p,\end{cases}
$$

for $A_{i} \in \mathbb{R}_{\text {skew }}^{2 \times 2}$ and $b_{i} \in \mathbb{R}^{2}, i=1,2$. Now repeating the calculation in (6.8) for the sets $P_{1}=\left\{x \in \Omega^{\prime}: x_{1}<p\right\}$ and $P_{2}=\Omega^{\prime} \backslash P_{1}$ we find $s, t \in \mathbb{R}$ such that for $x \in \Omega$ almost everywhere we have

$$
\begin{aligned}
\bar{u}(x):=\lim _{k \rightarrow \infty} \varepsilon_{k}^{-\frac{1}{2}}\left(y_{\varepsilon_{k}}(x)-x\right)= & u(x)-\left(A_{1} x+b_{1}\right) \chi_{x_{1}<p}(x) \\
& -\left(A_{2} x+b_{2}\right) \chi_{x_{1}>p}(x) \\
& +(0, s) \chi_{x_{1}<p}(x)+((l a, t)) \chi_{x_{1}>p}(x) .
\end{aligned}
$$

Then $\bar{u}$ satisfies Theorem 2.12(ii). This concludes the proof.

Acknowledgements. Open access funding provided by University of Vienna. I am very grateful to BERND SCHMIDT for many stimulating discussions and valuable comments from which the results of this paper and their exposition have benefited a lot. Moreover, I am gratefully indebted to the referee for her/his careful reading of the manuscript and many helpful suggestions.

Open Access This article is distributed under the terms of the Creative Commons Attribution 4.0 International License (http://creativecommons.org/licenses/by/4.0/), which permits unrestricted use, distribution, and reproduction in any medium, provided you give appropriate credit to the original author(s) and the source, provide a link to the Creative Commons license, and indicate if changes were made. 


\section{References}

1. Ambrosio, L.: Existence theory for a new class of variational problems. Arch. Ration. Mech. Anal. 111 (1990), 291-322

2. Ambrosio, L., Coscia, A., Dal Maso, G.: Fine properties of functions with bounded deformation. Arch. Ration. Mech. Anal. 139 (1997), 201-238

3. Ambrosio, L., Fusco, N., Pallara, D.: Functions of Bounded Variation and Free Discontinuity Problems. Oxford University Press, Oxford, 2000

4. Bellettini, G., Coscia, A., Dal Maso, G.: Compactness and lower semicontinuity properties in $S B D(\Omega)$. Math. Z. 228 (1998), 337-351

5. Bourdin, B.: Numerical implementation of the variational formulation for quasi-static brittle fracture. Interfaces Free Bound. 9 (2007), 411-430

6. Bourdin, B., Francfort, G.A., Marigo, J.J.: Numerical experiments in revisited brittle fracture. J. Mech. Phys. Solids 48 (2000), 797-826

7. Bourdin, B., Francfort, G.A., Marigo, J.J.: The variational approach to fracture. $J$. Elast. 91 (2008), 5-148.

8. Braides, A., Dal Maso, G., Garroni, A.: Variational formulation of softening phenomena in fracture mechanics. The one-dimensional case. Arch. Ration. Mech. Anal. 146 (1999), 23-58

9. Braides, A., Gelli, M.S.: Limits of discrete systems with long-range interactions. $J$. Convex Anal. 9 (2002), 363-399

10. Braides, A., Lew, A., Ortiz, M.: Effective cohesive behavior of layers of interatomic planes. Arch. Ration. Mech. Anal. 180 (2006), 151-182

11. Braides, A., Solci, M., Vitali, E.: A derivation of linear elastic energies from pairinteraction atomistic systems. Netw. Heterog. Media 2 (2007), 551-567

12. Burke, S., Ortner, C., Süli, E.: An adaptive finite element approximation of a generalized Ambrosio-Tortorelli functional. Math. Models Methods Appl. Sci. 23 (2013), $1663-1697$

13. Chambolle, A.: A density result in two-dimensional linearized elasticity, and applications. Arch. Ration. Mech. Anal. 167 (2003), 167-211

14. Chambolle, A.: An approximation result for special functions with bounded deformation. J. Math. Pures Appl. 83 (2004), 929-954

15. Сhambolle, A., Giacomini, A., Ponsiglione, M.: Piecewise rigidity. J. Funct. Anal. 244 (2007), 134-153.

16. Cortesani, G., Toader, R.: A density result in SBV with respect to non-isotropic energies. Nonlinear Analysis. 38 (1999), 585-604

17. Dal Maso, G.: An introduction to $\Gamma$-convergence. Birkhäuser, Boston - Basel - Berlin. 1993

18. Dal Maso, G.: Generalized functions of bounded deformation. J. Eur. Math. Soc. (JEMS). 15 (2013), 1943-1997

19. Dal Maso, G., Francfort, G.A., Toader, R.: Quasistatic crack growth in nonlinear elasticity. Arch. Ration. Mech. Anal. 176 (2005), 165-225

20. Dal Maso, G., Francfort, G.A., Toader, R.: Quasi-static crack evolution in brittle fracture: the case of bounded solutions. Calculus of Variations. Topics from the Mathematical Heritage of Ennio De Giorgi, 247-266, Quaderni di Matematica, Dipartimento di Matematica della Seconda Università di Napoli, Vol. 14, 2004

21. Dal Maso, G., Negri, M., Percivale, D.: Linearized elasticity as $\Gamma$-limit of finite elasticity. Set-valued Anal. 10 (2002), 165-183

22. Dal Maso, G., Lazzaroni, G.: Quasistatic crack growth in finite elasticity with noninterpenetration. Ann. Inst. H. Poincaré Anal. Non Linéaire. 27 (2010), 257-290

23. De Giorgi, E., Ambrosio, L.: Un nuovo funzionale del calcolo delle variazioni. Acc. Naz. Lincei, Rend. Cl. Sci. Fis. Mat. Natur. 82 (1988), 199-210

24. Dokтor, P.: Approximation of domains with Lipschitzian boundary. Čas. Pěst. Mat. 101 (1976), 237-255

25. Federer, H.: Geometric measure theory. Springer, New York, 1969 
26. Focardi, M., Iurlano, F.: Asymptotic analysis of Ambrosio- Tortorelli energies in linearized elasticity. SIAM J. Math. Anal. 46 (2014), 2936-2955

27. Francfort, G.A., Marigo, J.J.: Revisiting brittle fracture as an energy minimization problem. J. Mech. Phys. Solids. 46 (1998), 1319-1342

28. Friedrich, M., Schmidt, B.: A quantitative geometric rigidity result in SBD. Preprint, 2015

29. Friedrich, M., Schmidt, B.: An atomistic-to-continuum analysis of crystal cleavage in a two-dimensional model problem. J. Nonlin. Sci. 24 (2014), 145-183

30. Friedrich, M., Schmidt, B.: An analysis of crystal cleavage in the passage from atomistic models to continuum theory. Arch. Ration. Mech. Anal. 217 (2015), 263-308

31. Friedrich, M., Schmidt, B.: On a discrete-to-continuum convergence result for a two dimensional brittle material in the small displacement regime. 10 (2015), 321-342

32. Friesecke, G., James, R.D., Müller, S.: A theorem on geometric rigidity and the derivation of nonlinear plate theory from three-dimensional elasticity. Comm. Pure Appl. Math. 55 (2002), 1461-1506

33. Griffith, A.A.: The phenomena of rupture and flow in solids. Philos. Trans. R. Soc. London. 221 (1921), 163-198

34. IURLANO, F.: A density result for GSBD and its application to the approximation of brittle fracture energies. Calc. Var. PDE. 51 (2014), 315-342

35. Kristensen, J.: Lower semicontinuity in spaces of weakly differentiable functions. Math. Ann. 313 (1999), 653-710

36. Miller, R.E., Tadmor, E.: The Quasicontinuum Method: Overview, applications and current directions. Journal of Computer-Aided Materials Design. 9 (2002), 203-239

37. Mora-Corral, C.: Explicit energy-minimizers of incompressible elastic brittle bars under uniaxial extension. C. R. Acad. Sci. Paris. 348 (2010), 1045-1048

38. NeGri, M.: Finite element approximation of the Griffith's model in fracture mechanics. Numer. Math. 95 (2003), 653-687

39. NeGri, M.: A non-local approximation of free discontinuity problems in SBV and SBD. Calc. Var. PDE. 25 (2005), 33-62

40. Negri, M., Toader, R.: Scaling in fracture mechanics by Bažant's law: from finite to linearized elasticity. Math. Models Methods Appl. Sci. 25 (2015), 1389-1420

41. Schmidt, B.: Linear $\Gamma$-limits of multiwell energies in nonlinear elasticity theory. Continuum Mech. Thermodyn. 20 (2008) 375-396

42. Schmidt, B.: On the derivation of linear elasticity from atomistic models. Netw. Heterog. Media. 4 (2009), 789-812

43. Schmidt, B., Fraternali, F., Ortiz, M.: Eigenfracture: an eigendeformation approach to variational fracture. SIAM Multiscale Model. Simul. 7 (2009), 1237-1266

44. Tadmor, E., Ortiz, M., Phillips, R.: Quasicontinuum analysis of defects in solids. Phil. Mag. A 73 (1996), 1529-1563

45. ZhANG, K.: An approximation theorem for sequences of linear strains and its applications. ESAIM Control Optim. Calc. Var. 10 (2004), 224-242

\section{MANUEl FRIEDRICH}

Faculty of Mathematics, University of Vienna, Oskar-Morgenstern-Platz 1, 1090 Vienna, Austria. e-mail: manuel.friedrich@univie.ac.at

(Received April 10, 2015 / Accepted March 15, 2017)

Published online March 23, 2017 - (C) The Author(s) (2017)

This article is an open access publication 